Network Working Group

Request for Comments: 2083

Category: Informational
T. Boutell, et. al. Boutell.Com, Inc. March 1997

\title{
PNG (Portable Network Graphics) Specification
}

Version 1.0

Status of this Memo

This memo provides information for the Internet community. This memo does not specify an Internet standard of any kind. Distribution of this memo is unlimited.

\section{IESG Note:}

The IESG takes no position on the validity of any Intellectual Property Rights statements contained in this document.

\section{Abstract}

This document describes PNG (Portable Network Graphics), an extensible file format for the lossless, portable, well-compressed storage of raster images. PNG provides a patent-free replacement for GIF and can also replace many common uses of TIFF. Indexed-color, grayscale, and truecolor images are supported, plus an optional alpha channel. Sample depths range from 1 to 16 bits.

PNG is designed to work well in online viewing applications, such as the World Wide Web, so it is fully streamable with a progressive display option. PNG is robust, providing both full file integrity checking and simple detection of common transmission errors. Also, PNG can store gamma and chromaticity data for improved color matching on heterogeneous platforms.

This specification defines the Internet Media Type image/png.

Table of Contents

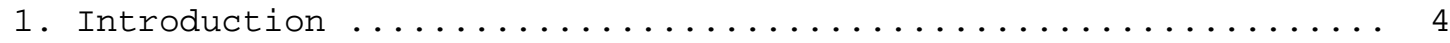

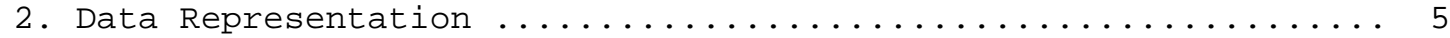

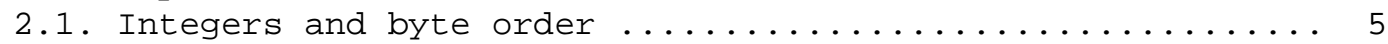

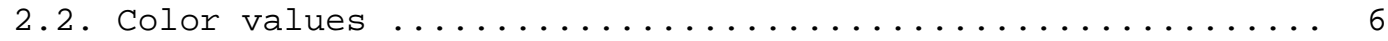

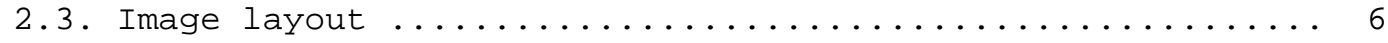

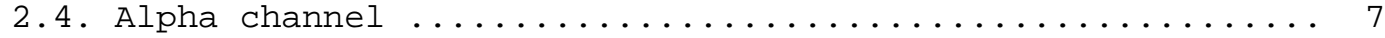

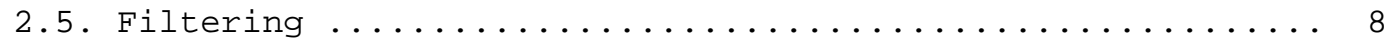

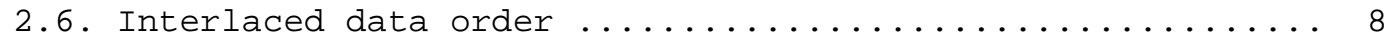

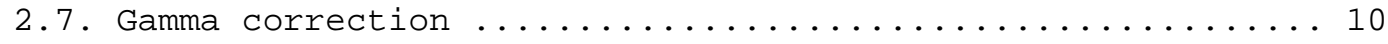




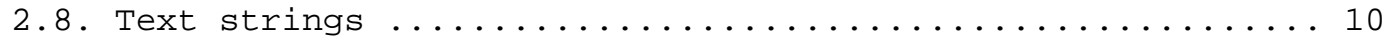

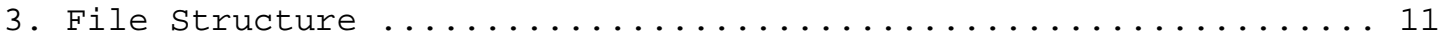

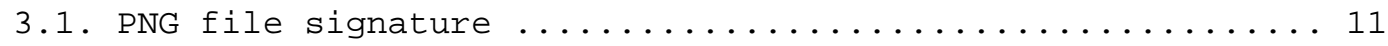

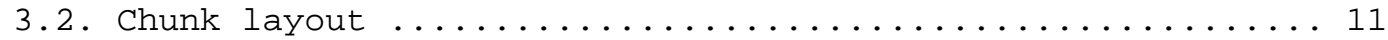

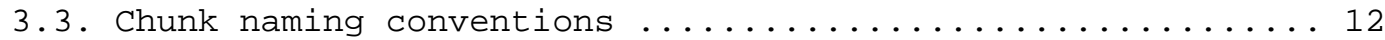

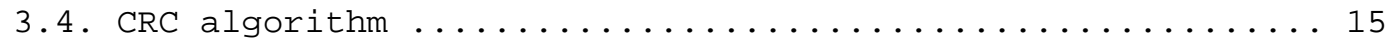

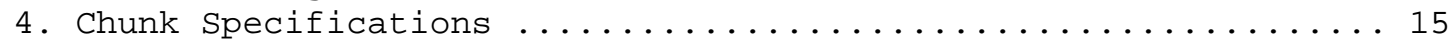

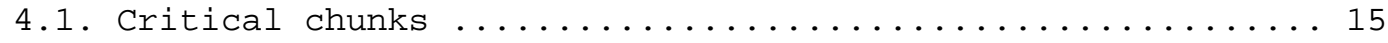

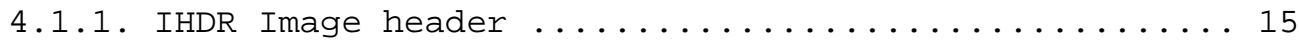

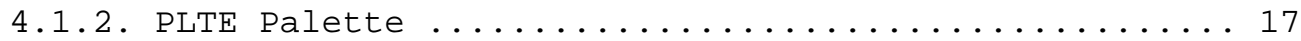

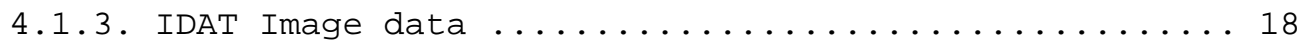

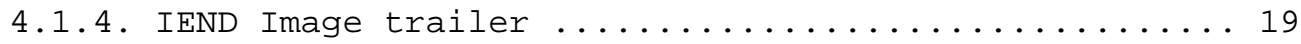

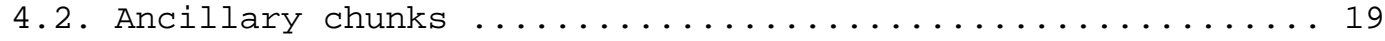

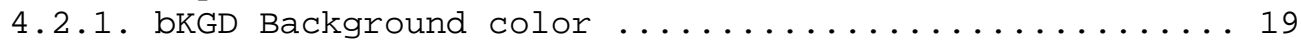

4.2.2. CHRM Primary chromaticities and white point ...... 20

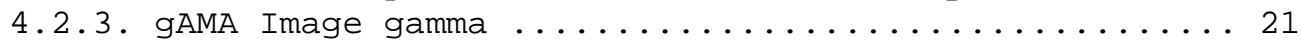

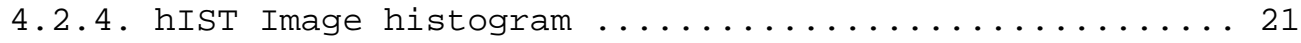

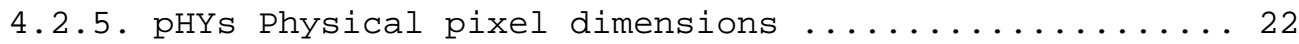

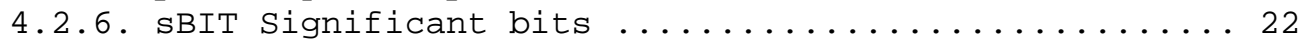

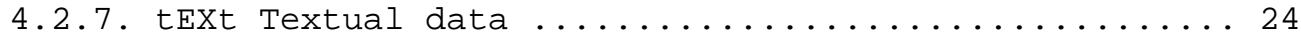

4.2.8. tIME Image last-modification time ............ 25

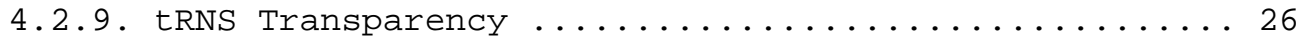

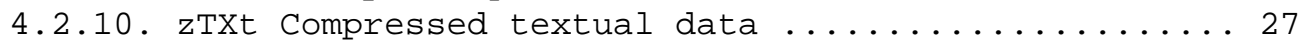

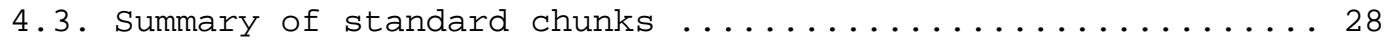

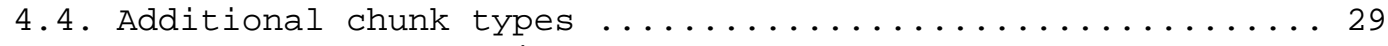

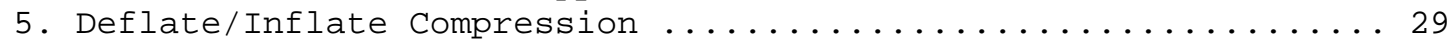

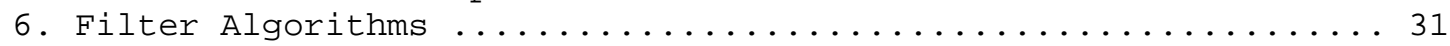

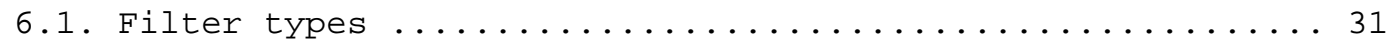

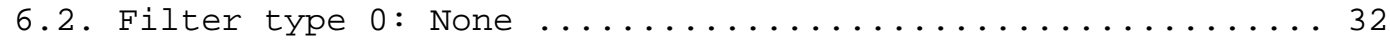

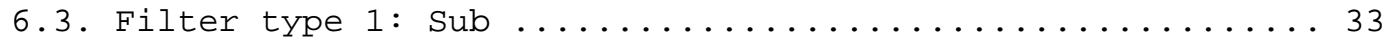

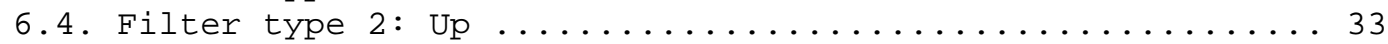

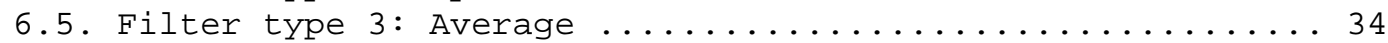

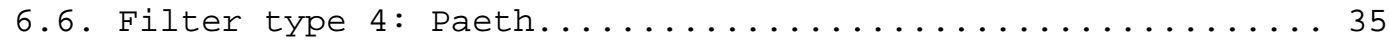

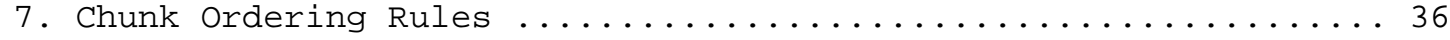

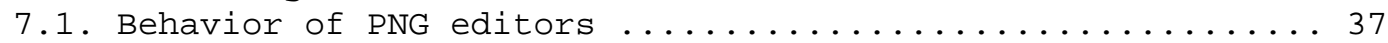

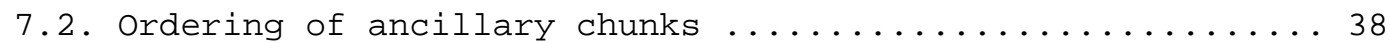

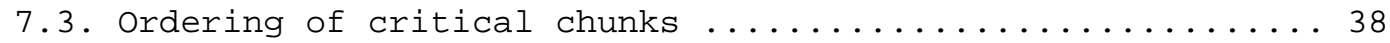

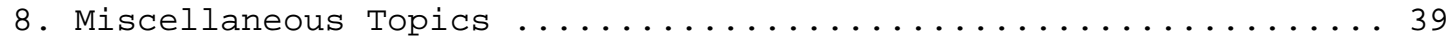

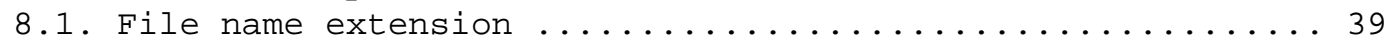

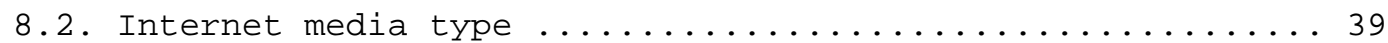

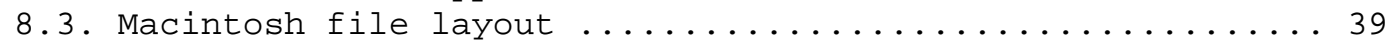

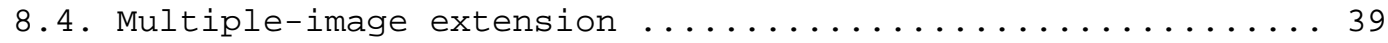

8.5. Security considerations ..................... 40

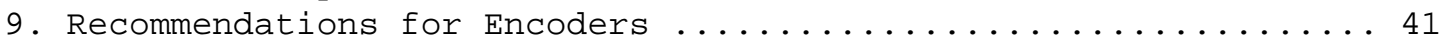

9.1. Sample depth scaling ....................... 41

9.2. Encoder gamma handling ...................... 42

9.3. Encoder color handling ...................... 45

9.4. Alpha channel creation ..................... 47 


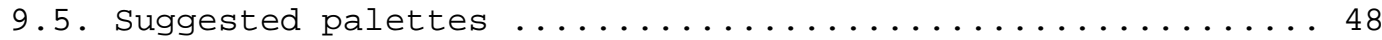

9.6. Filter selection .......................... 49

9.7. Text chunk processing ....................... 49

9.8. Use of private chunks ...................... 50

9.9. Private type and method codes .................. 51

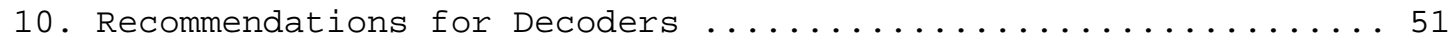

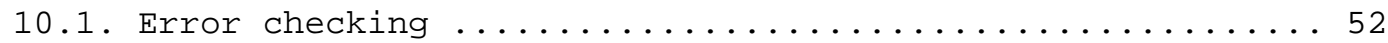

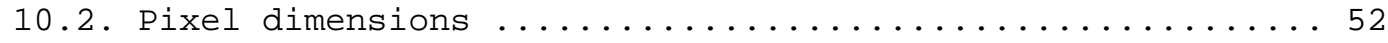

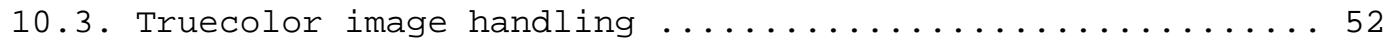

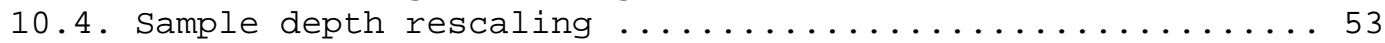

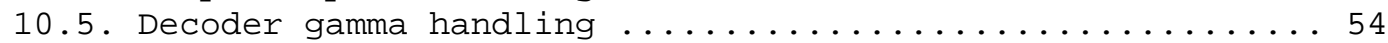

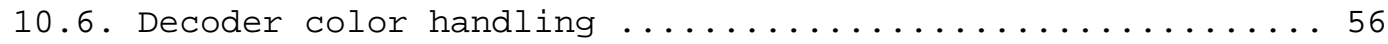

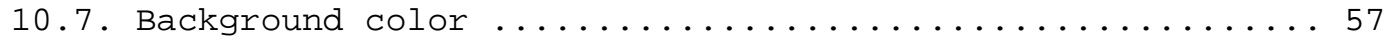

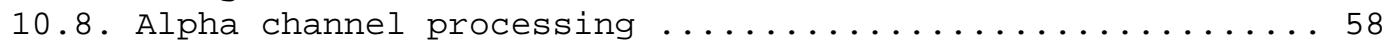

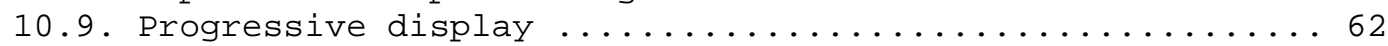

10.10. Suggested-palette and histogram usage ...........63

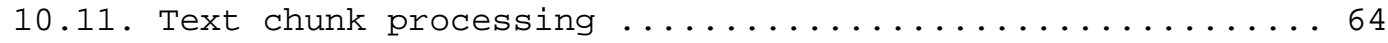

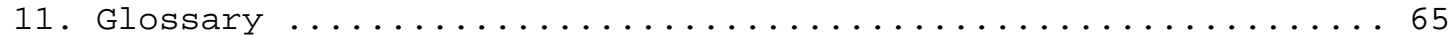

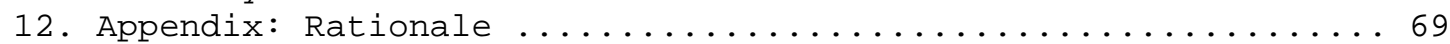

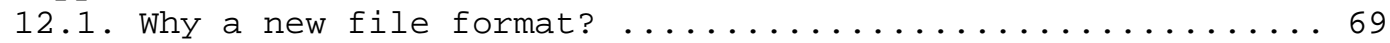

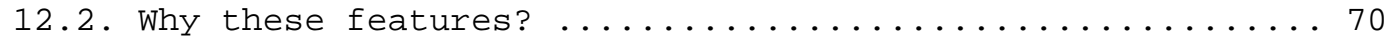

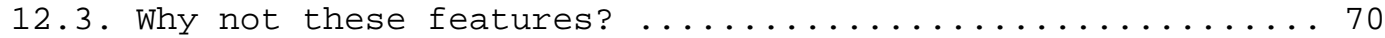

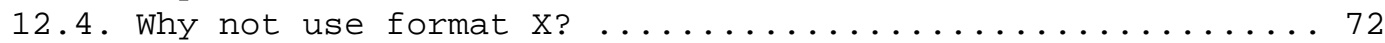

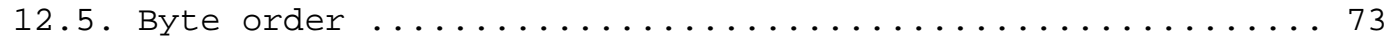

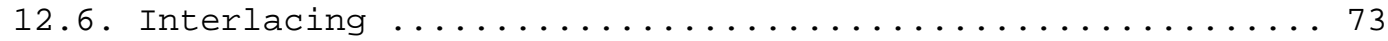

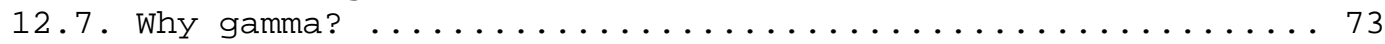

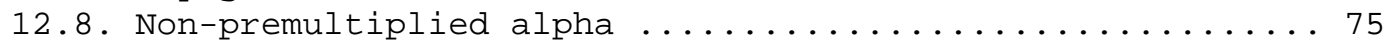

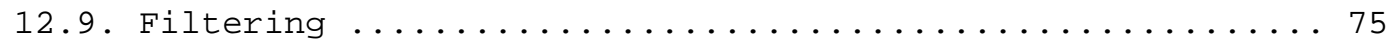

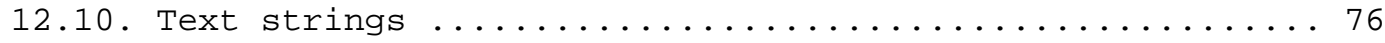

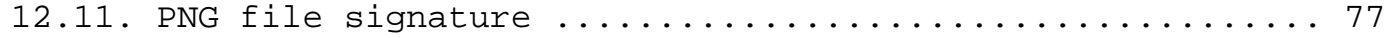

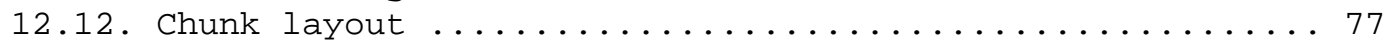

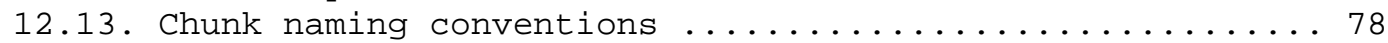

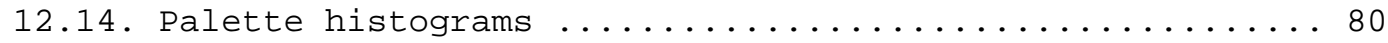

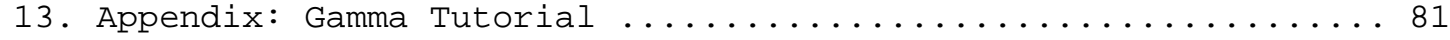

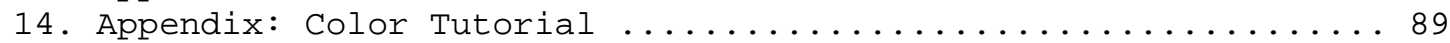

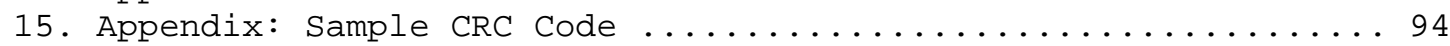

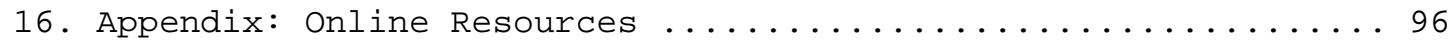

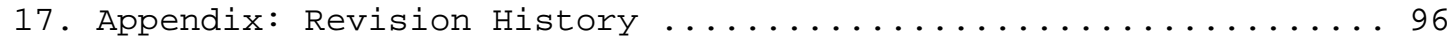

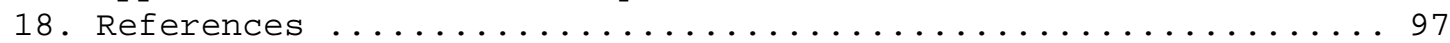

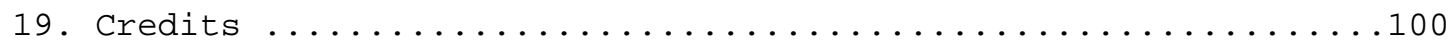




\section{Introduction}

The PNG format provides a portable, legally unencumbered, wellcompressed, well-specified standard for lossless bitmapped image files.

Although the initial motivation for developing PNG was to replace GIF, the design provides some useful new features not available in GIF, with minimal cost to developers.

GIF features retained in PNG include:

* Indexed-color images of up to 256 colors.

* Streamability: files can be read and written serially, thus allowing the file format to be used as a communications protocol for on-the-fly generation and display of images.

* Progressive display: a suitably prepared image file can be displayed as it is received over a communications link, yielding a low-resolution image very quickly followed by gradual improvement of detail.

* Transparency: portions of the image can be marked as transparent, creating the effect of a non-rectangular image.

* Ancillary information: textual comments and other data can be stored within the image file.

* Complete hardware and platform independence.

* Effective, 100\% lossless compression.

Important new features of PNG, not available in GIF, include:

* Truecolor images of up to 48 bits per pixel.

* Grayscale images of up to 16 bits per pixel.

* Full alpha channel (general transparency masks).

* Image gamma information, which supports automatic display of images with correct brightness/contrast regardless of the machines used to originate and display the image.

* Reliable, straightforward detection of file corruption.

* Faster initial presentation in progressive display mode.

PNG is designed to be:

* Simple and portable: developers should be able to implement PNG easily.

* Legally unencumbered: to the best knowledge of the PNG authors, no algorithms under legal challenge are used. (Some considerable effort has been spent to verify this.)

* Well compressed: both indexed-color and truecolor images are compressed as effectively as in any other widely used lossless format, and in most cases more effectively. 
* Interchangeable: any standard-conforming PNG decoder must read all conforming PNG files.

* Flexible: the format allows for future extensions and private add-ons, without compromising interchangeability of basic PNG.

* Robust: the design supports full file integrity checking as well as simple, quick detection of common transmission errors.

The main part of this specification gives the definition of the file format and recommendations for encoder and decoder behavior. An appendix gives the rationale for many design decisions. Although the rationale is not part of the formal specification, reading it can help implementors understand the design. Cross-references in the main text point to relevant parts of the rationale. Additional appendixes, also not part of the formal specification, provide tutorials on gamma and color theory as well as other supporting material.

In this specification, the word "must" indicates a mandatory requirement, while "should" indicates recommended behavior.

See Rationale: Why a new file format? (Section 12.1), Why these features? (Section 12.2), Why not these features? (Section 12.3), Why not use format $X$ ? (Section 12.4).

Pronunciation

PNG is pronounced "ping".

2. Data Representation

This chapter discusses basic data representations used in PNG files, as well as the expected representation of the image data.

\subsection{Integers and byte order}

All integers that require more than one byte must be in network byte order: the most significant byte comes first, then the less significant bytes in descending order of significance (MSB LSB for two-byte integers, B3 B2 B1 B0 for four-byte integers). The highest bit (value 128) of a byte is numbered bit 7; the lowest bit (value 1) is numbered bit 0 . Values are unsigned unless otherwise noted. Values explicitly noted as signed are represented in two's complement notation.

See Rationale: Byte order (Section 12.5). 


\subsection{Color values}

Colors can be represented by either grayscale or RGB (red, green, blue) sample data. Grayscale data represents luminance; RGB data represents calibrated color information (if the cHRM chunk is present) or uncalibrated device-dependent color (if cHRM is absent). All color values range from zero (representing black) to most intense at the maximum value for the sample depth. Note that the maximum value at a given sample depth is (2^sampledepth) -1 , not $2^{\wedge}$ sampledepth.

Sample values are not necessarily linear; the gAMA chunk specifies the gamma characteristic of the source device, and viewers are strongly encouraged to compensate properly. See Gamma correction (Section 2.7).

Source data with a precision not directly supported in PNG (for example, 5 bit/sample truecolor) must be scaled up to the next higher supported bit depth. This scaling is reversible with no loss of data, and it reduces the number of cases that decoders have to cope with. See Recommendations for Encoders: Sample depth scaling (Section 9.1) and Recommendations for Decoders: Sample depth rescaling (Section 10.4).

\subsection{Image layout}

Conceptually, a PNG image is a rectangular pixel array, with pixels appearing left-to-right within each scanline, and scanlines appearing top-to-bottom. (For progressive display purposes, the data may actually be transmitted in a different order; see Interlaced data order, Section 2.6.) The size of each pixel is determined by the bit depth, which is the number of bits per sample in the image data.

Three types of pixel are supported:

* An indexed-color pixel is represented by a single sample that is an index into a supplied palette. The image bit depth determines the maximum number of palette entries, but not the color precision within the palette.

* A grayscale pixel is represented by a single sample that is a grayscale level, where zero is black and the largest value for the bit depth is white.

* A truecolor pixel is represented by three samples: red (zero $=$ black, $\max =$ red) appears first, then green (zero = black, $\max =$ green $)$, then blue (zero = black, $\max =\mathrm{blue})$. The bit depth specifies the size of each sample, not the total pixel size. 
Optionally, grayscale and truecolor pixels can also include an alpha sample, as described in the next section.

Pixels are always packed into scanlines with no wasted bits between pixels. Pixels smaller than a byte never cross byte boundaries; they are packed into bytes with the leftmost pixel in the high-order bits of a byte, the rightmost in the low-order bits. Permitted bit depths and pixel types are restricted so that in all cases the packing is simple and efficient.

PNG permits multi-sample pixels only with 8- and 16-bit samples, so multiple samples of a single pixel are never packed into one byte. 16-bit samples are stored in network byte order (MSB first).

Scanlines always begin on byte boundaries. When pixels have fewer than 8 bits and the scanline width is not evenly divisible by the number of pixels per byte, the low-order bits in the last byte of each scanline are wasted. The contents of these wasted bits are unspecified.

An additional "filter type" byte is added to the beginning of every scanline (see Filtering, Section 2.5). The filter type byte is not considered part of the image data, but it is included in the datastream sent to the compression step.

\subsection{Alpha channel}

An alpha channel, representing transparency information on a perpixel basis, can be included in grayscale and truecolor PNG images.

An alpha value of zero represents full transparency, and a value of (2^bitdepth)-1 represents a fully opaque pixel. Intermediate values indicate partially transparent pixels that can be combined with a background image to yield a composite image. (Thus, alpha is really the degree of opacity of the pixel. But most people refer to alpha as providing transparency information, not opacity information, and we continue that custom here.)

Alpha channels can be included with images that have either 8 or 16 bits per sample, but not with images that have fewer than 8 bits per sample. Alpha samples are represented with the same bit depth used for the image samples. The alpha sample for each pixel is stored immediately following the grayscale or RGB samples of the pixel. 
The color values stored for a pixel are not affected by the alpha value assigned to the pixel. This rule is sometimes called "unassociated" or "non-premultiplied" alpha. (Another common technique is to store sample values premultiplied by the alpha fraction; in effect, such an image is already composited against a black background. PNG does not use premultiplied alpha.)

Transparency control is also possible without the storage cost of a full alpha channel. In an indexed-color image, an alpha value can be defined for each palette entry. In grayscale and truecolor images, a single pixel value can be identified as being "transparent". These techniques are controlled by the tRNS ancillary chunk type.

If no alpha channel nor tRNS chunk is present, all pixels in the image are to be treated as fully opaque.

Viewers can support transparency control partially, or not at all. See Rationale: Non-premultiplied alpha (Section 12.8), Recommendations for Encoders: Alpha channel creation (Section 9.4), and Recommendations for Decoders: Alpha channel processing (Section 10.8).

\subsection{Filtering}

PNG allows the image data to be filtered before it is compressed. Filtering can improve the compressibility of the data. The filter step itself does not reduce the size of the data. All PNG filters are strictly lossless.

PNG defines several different filter algorithms, including "None" which indicates no filtering. The filter algorithm is specified for each scanline by a filter type byte that precedes the filtered scanline in the precompression datastream. An intelligent encoder can switch filters from one scanline to the next. The method for choosing which filter to employ is up to the encoder.

See Filter Algorithms (Chapter 6) and Rationale: Filtering (Section 12.9).

\subsection{Interlaced data order}

A PNG image can be stored in interlaced order to allow progressive display. The purpose of this feature is to allow images to "fade in" when they are being displayed on-the-fly. Interlacing slightly expands the file size on average, but it gives the user a meaningful display much more rapidly. Note that decoders are 
required to be able to read interlaced images, whether or not they actually perform progressive display.

With interlace method 0 , pixels are stored sequentially from left to right, and scanlines sequentially from top to bottom (no interlacing).

Interlace method 1, known as Adam7 after its author, Adam M. Costello, consists of seven distinct passes over the image. Each pass transmits a subset of the pixels in the image. The pass in which each pixel is transmitted is defined by replicating the following 8-by-8 pattern over the entire image, starting at the upper left corner:

$\begin{array}{llllllll}1 & 6 & 4 & 6 & 2 & 6 & 4 & 6 \\ 7 & 7 & 7 & 7 & 7 & 7 & 7 & 7 \\ 5 & 6 & 5 & 6 & 5 & 6 & 5 & 6 \\ 7 & 7 & 7 & 7 & 7 & 7 & 7 & 7 \\ 3 & 6 & 4 & 6 & 3 & 6 & 4 & 6 \\ 7 & 7 & 7 & 7 & 7 & 7 & 7 & 7 \\ 5 & 6 & 5 & 6 & 5 & 6 & 5 & 6 \\ 7 & 7 & 7 & 7 & 7 & 7 & 7 & 7\end{array}$

Within each pass, the selected pixels are transmitted left to right within a scanline, and selected scanlines sequentially from top to bottom. For example, pass 2 contains pixels 4, 12, 20, etc. of scanlines 0, 8, 16, etc. (numbering from 0,0 at the upper left corner). The last pass contains the entirety of scanlines 1 , 3,5 , etc.

The data within each pass is laid out as though it were a complete image of the appropriate dimensions. For example, if the complete image is 16 by 16 pixels, then pass 3 will contain two scanlines, each containing four pixels. When pixels have fewer than 8 bits, each such scanline is padded as needed to fill an integral number of bytes (see Image layout, Section 2.3). Filtering is done on this reduced image in the usual way, and a filter type byte is transmitted before each of its scanlines (see Filter Algorithms, Chapter 6). Notice that the transmission order is defined so that all the scanlines transmitted in a pass will have the same number of pixels; this is necessary for proper application of some of the filters.

Caution: If the image contains fewer than five columns or fewer than five rows, some passes will be entirely empty. Encoders and decoders must handle this case correctly. In particular, filter type bytes are only associated with nonempty scanlines; no filter type bytes are present in an empty pass. 
See Rationale: Interlacing (Section 12.6) and Recommendations for Decoders: Progressive display (Section 10.9).

\subsection{Gamma correction}

PNG images can specify, via the gAMA chunk, the gamma characteristic of the image with respect to the original scene. Display programs are strongly encouraged to use this information, plus information about the display device they are using and room lighting, to present the image to the viewer in a way that reproduces what the image's original author saw as closely as possible. See Gamma Tutorial (Chapter 13) if you aren't already familiar with gamma issues.

Gamma correction is not applied to the alpha channel, if any. Alpha samples always represent a linear fraction of full opacity.

For high-precision applications, the exact chromaticity of the RGB data in a PNG image can be specified via the cHRM chunk, allowing more accurate color matching than gamma correction alone will provide. See Color Tutorial (Chapter 14) if you aren't already familiar with color representation issues.

See Rationale: Why gamma? (Section 12.7), Recommendations for Encoders: Encoder gamma handling (Section 9.2), and Recommendations for Decoders: Decoder gamma handling (Section $10.5)$.

\subsection{Text strings}

A PNG file can store text associated with the image, such as an image description or copyright notice. Keywords are used to indicate what each text string represents.

ISO 8859-1 (Latin-1) is the character set recommended for use in text strings [ISO-8859]. This character set is a superset of 7bit ASCII.

Character codes not defined in Latin-1 should not be used, because they have no platform-independent meaning. If a non-Latin-1 code does appear in a PNG text string, its interpretation will vary across platforms and decoders. Some systems might not even be able to display all the characters in Latin-1, but most modern systems can.

Provision is also made for the storage of compressed text. See Rationale: Text strings (Section 12.10). 
3. File structure

A PNG file consists of a PNG signature followed by a series of chunks. This chapter defines the signature and the basic properties of chunks. Individual chunk types are discussed in the next chapter.

3.1. PNG file signature

The first eight bytes of a PNG file always contain the following (decimal) values:

$\begin{array}{lllllllll}137 & 80 & 78 & 71 & 13 & 10 & 26 & 10\end{array}$

This signature indicates that the remainder of the file contains a single PNG image, consisting of a series of chunks beginning with an IHDR chunk and ending with an IEND chunk.

See Rationale: PNG file signature (Section 12.11).

3.2. Chunk layout

Each chunk consists of four parts:

Length

A 4-byte unsigned integer giving the number of bytes in the chunk's data field. The length counts only the data field, not itself, the chunk type code, or the CRC. Zero is a valid length. Although encoders and decoders should treat the length as unsigned, its value must not exceed (2^31)-1 bytes.

Chunk Type

A 4-byte chunk type code. For convenience in description and in examining $\mathrm{PNG}$ files, type codes are restricted to consist of uppercase and lowercase ASCII letters (A-z and $a-z$, or 65-90 and 97-122 decimal). However, encoders and decoders must treat the codes as fixed binary values, not character strings. For example, it would not be correct to represent the type code IDAT by the EBCDIC equivalents of those letters. Additional naming conventions for chunk types are discussed in the next section.

Chunk Data

The data bytes appropriate to the chunk type, if any. This field can be of zero length. 
CRC

A 4-byte CRC (Cyclic Redundancy Check) calculated on the preceding bytes in the chunk, including the chunk type code and chunk data fields, but not including the length field. The CRC is always present, even for chunks containing no data. See CRC algorithm (Section 3.4).

The chunk data length can be any number of bytes up to the maximum; therefore, implementors cannot assume that chunks are aligned on any boundaries larger than bytes.

Chunks can appear in any order, subject to the restrictions placed on each chunk type. (One notable restriction is that IHDR must appear first and IEND must appear last; thus the IEND chunk serves as an end-of-file marker.) Multiple chunks of the same type can appear, but only if specifically permitted for that type.

See Rationale: Chunk layout (Section 12.12).

\subsection{Chunk naming conventions}

Chunk type codes are assigned so that a decoder can determine some properties of a chunk even when it does not recognize the type code. These rules are intended to allow safe, flexible extension of the PNG format, by allowing a decoder to decide what to do when it encounters an unknown chunk. The naming rules are not normally of interest when the decoder does recognize the chunk's type.

Four bits of the type code, namely bit 5 (value 32) of each byte, are used to convey chunk properties. This choice means that a human can read off the assigned properties according to whether each letter of the type code is uppercase (bit 5 is 0 ) or lowercase (bit 5 is 1 ). However, decoders should test the properties of an unknown chunk by numerically testing the specified bits; testing whether a character is uppercase or lowercase is inefficient, and even incorrect if a locale-specific case definition is used.

It is worth noting that the property bits are an inherent part of the chunk name, and hence are fixed for any chunk type. Thus, TEXT and Text would be unrelated chunk type codes, not the same chunk with different properties. Decoders must recognize type codes by a simple four-byte literal comparison; it is incorrect to perform case conversion on type codes. 
The semantics of the property bits are:

Ancillary bit: bit 5 of first byte

0 (uppercase) = critical, 1 (lowercase) = ancillary.

Chunks that are not strictly necessary in order to meaningfully display the contents of the file are known as "ancillary" chunks. A decoder encountering an unknown chunk in which the ancillary bit is 1 can safely ignore the chunk and proceed to display the image. The time chunk (tIME) is an example of an ancillary chunk.

Chunks that are necessary for successful display of the file's contents are called "critical" chunks. A decoder encountering an unknown chunk in which the ancillary bit is 0 must indicate to the user that the image contains information it cannot safely interpret. The image header chunk (IHDR) is an example of a critical chunk.

Private bit: bit 5 of second byte 0 (uppercase) = public, 1 (lowercase) $=$ private.

A public chunk is one that is part of the PNG specification or is registered in the list of PNG special-purpose public chunk types. Applications can also define private (unregistered) chunks for their own purposes. The names of private chunks must have a lowercase second letter, while public chunks will always be assigned names with uppercase second letters. Note that decoders do not need to test the private-chunk property bit, since it has no functional significance; it is simply an administrative convenience to ensure that public and private chunk names will not conflict. See Additional chunk types (Section 4.4) and Recommendations for Encoders: Use of private chunks (Section 9.8).

Reserved bit: bit 5 of third byte Must be 0 (uppercase) in files conforming to this version of PNG.

The significance of the case of the third letter of the chunk name is reserved for possible future expansion. At the present time all chunk names must have uppercase third letters. (Decoders should not complain about a lowercase third letter, however, as some future version of the PNG specification could define a meaning for this bit. It is sufficient to treat a chunk with a lowercase third letter in the same way as any other unknown chunk type.) 
Safe-to-copy bit: bit 5 of fourth byte

0 (uppercase) = unsafe to copy, 1 (lowercase) $=$ safe to copy.

This property bit is not of interest to pure decoders, but it is needed by PNG editors (programs that modify PNG files). This bit defines the proper handling of unrecognized chunks in a file that is being modified.

If a chunk's safe-to-copy bit is 1, the chunk may be copied to a modified PNG file whether or not the software recognizes the chunk type, and regardless of the extent of the file modifications.

If a chunk's safe-to-copy bit is 0 , it indicates that the chunk depends on the image data. If the program has made any changes to critical chunks, including addition, modification, deletion, or reordering of critical chunks, then unrecognized unsafe chunks must not be copied to the output PNG file. (Of course, if the program does recognize the chunk, it can choose to output an appropriately modified version.)

A PNG editor is always allowed to copy all unrecognized chunks if it has only added, deleted, modified, or reordered ancillary chunks. This implies that it is not permissible for ancillary chunks to depend on other ancillary chunks.

PNG editors that do not recognize a critical chunk must report an error and refuse to process that PNG file at all. The safe/unsafe mechanism is intended for use with ancillary chunks. The safe-to-copy bit will always be 0 for critical chunks.

Rules for PNG editors are discussed further in Chunk Ordering Rules (Chapter 7).

For example, the hypothetical chunk type name "bLob" has the property bits:

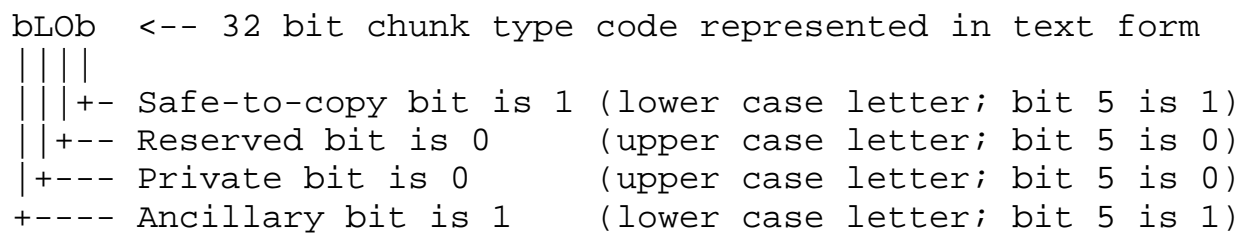

Therefore, this name represents an ancillary, public, safe-to-copy chunk. 
See Rationale: Chunk naming conventions (Section 12.13).

3.4. CRC algorithm

Chunk CRCs are calculated using standard CRC methods with pre and post conditioning, as defined by ISO 3309 [ISO-3309] or ITU-T V.42 [ITU-V42]. The CRC polynomial employed is

$$
x^{\wedge} 32+x^{\wedge} 26+x^{\wedge} 23+x^{\wedge} 22+x^{\wedge} 16+x^{\wedge} 12+x^{\wedge} 11+x^{\wedge} 10+x^{\wedge} 8+x^{\wedge} 7+x^{\wedge} 5+x^{\wedge} 4+x^{\wedge} 2+x+1
$$

The 32-bit CRC register is initialized to all 1's, and then the data from each byte is processed from the least significant bit (1) to the most significant bit (128). After all the data bytes are processed, the $\mathrm{CRC}$ register is inverted (its ones complement is taken). This value is transmitted (stored in the file) MSB first. For the purpose of separating into bytes and ordering, the least significant bit of the 32-bit CRC is defined to be the coefficient of the $\mathrm{x}^{\wedge} 31$ term.

Practical calculation of the CRC always employs a precalculated table to greatly accelerate the computation. See Sample CRC Code (Chapter 15).

4. Chunk Specifications

This chapter defines the standard types of PNG chunks.

4.1. Critical chunks

All implementations must understand and successfully render the standard critical chunks. A valid PNG image must contain an IHDR chunk, one or more IDAT chunks, and an IEND chunk.

4.1.1. IHDR Image header

The IHDR chunk must appear FIRST. It contains:

$\begin{array}{ll}\text { Width: } & 4 \text { bytes } \\ \text { Height: } & 4 \text { bytes } \\ \text { Bit depth: } & 1 \text { byte } \\ \text { Color type: } & 1 \text { byte } \\ \text { Compression method: } & 1 \text { byte } \\ \text { Filter method: } & 1 \text { byte } \\ \text { Interlace method: } & 1 \text { byte }\end{array}$


Width and height give the image dimensions in pixels. They are 4-byte integers. Zero is an invalid value. The maximum for each is (2^31)-1 in order to accommodate languages that have difficulty with unsigned 4-byte values.

Bit depth is a single-byte integer giving the number of bits per sample or per palette index (not per pixel). Valid values are 1, 2, 4, 8, and 16, although not all values are allowed for all color types.

Color type is a single-byte integer that describes the interpretation of the image data. Color type codes represent sums of the following values: 1 (palette used), 2 (color used), and 4 (alpha channel used). Valid values are 0, 2, 3, 4, and 6.

Bit depth restrictions for each color type are imposed to simplify implementations and to prohibit combinations that do not compress well. Decoders must support all legal combinations of bit depth and color type. The allowed combinations are:

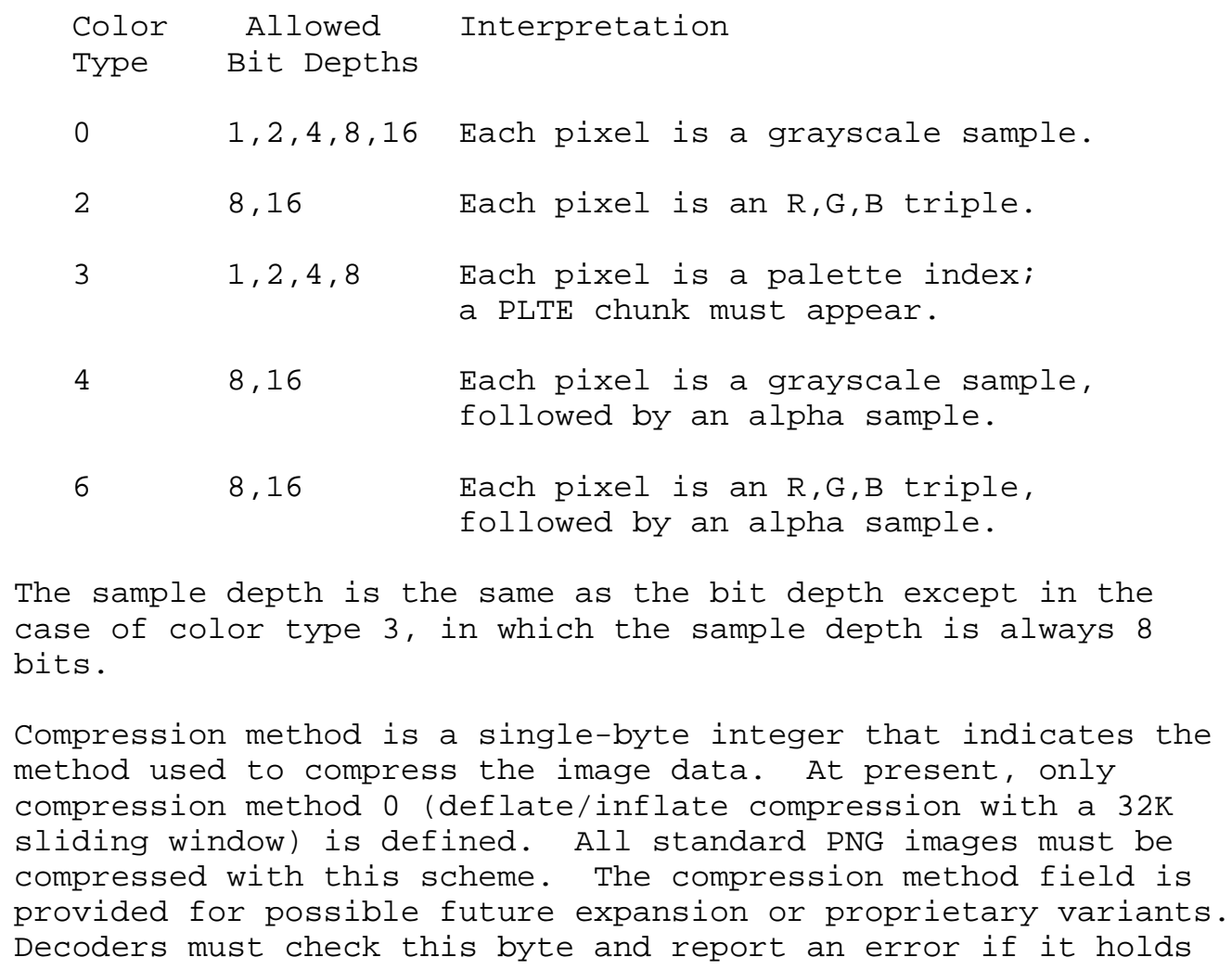


an unrecognized code. See Deflate/Inflate Compression (Chapter 5) for details.

Filter method is a single-byte integer that indicates the preprocessing method applied to the image data before compression. At present, only filter method 0 (adaptive filtering with five basic filter types) is defined. As with the compression method field, decoders must check this byte and report an error if it holds an unrecognized code. See Filter Algorithms (Chapter 6) for details.

Interlace method is a single-byte integer that indicates the transmission order of the image data. Two values are currently defined: 0 (no interlace) or 1 (Adam7 interlace). See Interlaced data order (Section 2.6) for details.

\subsubsection{PLTE Palette}

The PLTE chunk contains from 1 to 256 palette entries, each a three-byte series of the form:

$$
\begin{array}{ll}
\text { Red: } & 1 \text { byte }(0=\text { black, } 255=\text { red }) \\
\text { Green: } 1 \text { byte }(0=b l a c k, 255=\text { green }) \\
\text { Blue: } 1 \text { byte }(0=\text { black, } 255=\text { blue })
\end{array}
$$

The number of entries is determined from the chunk length. A chunk length not divisible by 3 is an error.

This chunk must appear for color type 3, and can appear for color types 2 and 6 ; it must not appear for color types 0 and 4. If this chunk does appear, it must precede the first IDAT chunk. There must not be more than one PLTE chunk.

For color type 3 (indexed color), the PLTE chunk is required. The first entry in PLTE is referenced by pixel value 0 , the second by pixel value 1 , etc. The number of palette entries must not exceed the range that can be represented in the image bit depth (for example, $2^{\wedge} 4=16$ for a bit depth of 4). It is permissible to have fewer entries than the bit depth would allow. In that case, any out-of-range pixel value found in the image data is an error.

For color types 2 and 6 (truecolor and truecolor with alpha), the PLTE chunk is optional. If present, it provides a suggested set of from 1 to 256 colors to which the truecolor image can be quantized if the viewer cannot display truecolor directly. If PLTE is not present, such a viewer will need to select colors on its own, but it is often preferable for this 
to be done once by the encoder. (See Recommendations for

Encoders: Suggested palettes, Section 9.5.)

Note that the palette uses 8 bits ( 1 byte) per sample

regardless of the image bit depth specification. In

particular, the palette is 8 bits deep even when it is a

suggested quantization of a 16-bit truecolor image.

There is no requirement that the palette entries all be used by the image, nor that they all be different.

4.1.3. IDAT Image data

The IDAT chunk contains the actual image data. To create this data:

* Begin with image scanlines represented as described in Image layout (Section 2.3); the layout and total size of this raw data are determined by the fields of IHDR.

* Filter the image data according to the filtering method specified by the IHDR chunk. (Note that with filter method 0 , the only one currently defined, this implies prepending a filter type byte to each scanline.)

* Compress the filtered data using the compression method specified by the IHDR chunk.

The IDAT chunk contains the output datastream of the compression algorithm.

To read the image data, reverse this process.

There can be multiple IDAT chunks; if so, they must appear consecutively with no other intervening chunks. The compressed datastream is then the concatenation of the contents of all the IDAT chunks. The encoder can divide the compressed datastream into IDAT chunks however it wishes. (Multiple IDAT chunks are allowed so that encoders can work in a fixed amount of memory; typically the chunk size will correspond to the encoder's buffer size.) It is important to emphasize that IDAT chunk boundaries have no semantic significance and can occur at any point in the compressed datastream. A PNG file in which each IDAT chunk contains only one data byte is legal, though remarkably wasteful of space. (For that matter, zero-length IDAT chunks are legal, though even more wasteful.)

See Filter Algorithms (Chapter 6) and Deflate/Inflate Compression (Chapter 5) for details. 


\subsubsection{IEND Image trailer}

The IEND chunk must appear LAST. It marks the end of the PNG datastream. The chunk's data field is empty.

\subsection{Ancillary chunks}

All ancillary chunks are optional, in the sense that encoders need not write them and decoders can ignore them. However, encoders are encouraged to write the standard ancillary chunks when the information is available, and decoders are encouraged to interpret these chunks when appropriate and feasible.

The standard ancillary chunks are listed in alphabetical order. This is not necessarily the order in which they would appear in a file.

\subsection{1. bKGD Background color}

The bKGD chunk specifies a default background color to present the image against. Note that viewers are not bound to honor this chunk; a viewer can choose to use a different background.

For color type 3 (indexed color), the bKGD chunk contains:

$$
\text { Palette index: } 1 \text { byte }
$$

The value is the palette index of the color to be used as background.

For color types 0 and 4 (grayscale, with or without alpha), bKGD contains:

$$
\text { Gray: } 2 \text { bytes, range } 0 \ldots\left(2^{\wedge} \text { bitdepth }\right)-1
$$

(For consistency, 2 bytes are used regardless of the image bit depth.) The value is the gray level to be used as background.

For color types 2 and 6 (truecolor, with or without alpha), bKGD contains:

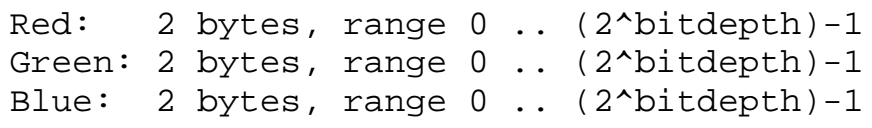

(For consistency, 2 bytes per sample are used regardless of the image bit depth.) This is the RGB color to be used as background. 
When present, the bKGD chunk must precede the first IDAT chunk, and must follow the PLTE chunk, if any.

See Recommendations for Decoders: Background color (Section $10.7)$.

4.2.2. cHRM Primary chromaticities and white point

Applications that need device-independent specification of colors in a PNG file can use the cHRM chunk to specify the 1931 CIE $x, y$ chromaticities of the red, green, and blue primaries used in the image, and the referenced white point. See color Tutorial (Chapter 14) for more information.

The cHRM chunk contains:

$\begin{array}{ll}\text { White Point } x: & 4 \text { bytes } \\ \text { White Point } y: & 4 \text { bytes } \\ \text { Red } x: & 4 \text { bytes } \\ \text { Red } y: & 4 \text { bytes } \\ \text { Green } x: & 4 \text { bytes } \\ \text { Green y: } & 4 \text { bytes } \\ \text { Blue } x: & 4 \text { bytes } \\ \text { Blue y: } & 4 \text { bytes }\end{array}$

Each value is encoded as a 4-byte unsigned integer, representing the $\mathrm{x}$ or $\mathrm{y}$ value times 100000 . For example, a value of 0.3127 would be stored as the integer 31270 .

cHRM is allowed in all PNG files, although it is of little value for grayscale images.

If the encoder does not know the chromaticity values, it should not write a cHRM chunk; the absence of a cHRM chunk indicates that the image's primary colors are device-dependent.

If the cHRM chunk appears, it must precede the first IDAT chunk, and it must also precede the PLTE chunk if present.

See Recommendations for Encoders: Encoder color handling (Section 9.3), and Recommendations for Decoders: Decoder color handling (Section 10.6). 
4.2.3. gAMA Image gamma

The gAMA chunk specifies the gamma of the camera (or simulated camera) that produced the image, and thus the gamma of the image with respect to the original scene. More precisely, the gAMA chunk encodes the file_gamma value, as defined in Gamma Tutorial (Chapter 13).

The gAMA chunk contains:

Image gamma: 4 bytes

The value is encoded as a 4-byte unsigned integer, representing gamma times 100000 . For example, a gamma of 0.45 would be stored as the integer 45000 .

If the encoder does not know the image's gamma value, it should not write a gAMA chunk; the absence of a gAMA chunk indicates that the gamma is unknown.

If the gAMA chunk appears, it must precede the first IDAT chunk, and it must also precede the PLTE chunk if present.

See Gamma correction (Section 2.7), Recommendations for

Encoders: Encoder gamma handling (Section 9.2), and Recommendations for Decoders: Decoder gamma handling (Section $10.5)$.

4.2.4. hIST Image histogram

The hIST chunk gives the approximate usage frequency of each color in the color palette. A histogram chunk can appear only when a palette chunk appears. If a viewer is unable to provide all the colors listed in the palette, the histogram may help it decide how to choose a subset of the colors for display.

The hIST chunk contains a series of 2-byte (16 bit) unsigned integers. There must be exactly one entry for each entry in the PLTE chunk. Each entry is proportional to the fraction of pixels in the image that have that palette index; the exact scale factor is chosen by the encoder.

Histogram entries are approximate, with the exception that a zero entry specifies that the corresponding palette entry is not used at all in the image. It is required that a histogram entry be nonzero if there are any pixels of that color. 
When the palette is a suggested quantization of a truecolor image, the histogram is necessarily approximate, since a decoder may map pixels to palette entries differently than the encoder did. In this situation, zero entries should not appear.

The hIST chunk, if it appears, must follow the PLTE chunk, and must precede the first IDAT chunk.

See Rationale: Palette histograms (Section 12.14), and Recommendations for Decoders: Suggested-palette and histogram usage (Section 10.10).

4.2.5. pHYs Physical pixel dimensions

The pHYs chunk specifies the intended pixel size or aspect ratio for display of the image. It contains:

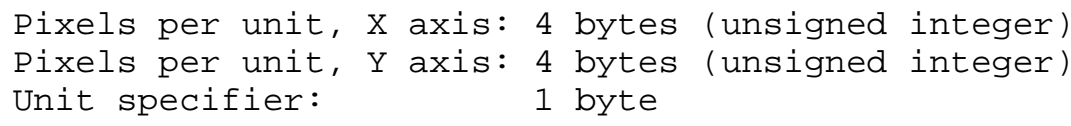

When the unit specifier is 0 , the pHYs chunk defines pixel aspect ratio only; the actual size of the pixels remains unspecified.

Conversion note: one inch is equal to exactly 0.0254 meters.

If this ancillary chunk is not present, pixels are assumed to be square, and the physical size of each pixel is unknown.

If present, this chunk must precede the first IDAT chunk.

See Recommendations for Decoders: Pixel dimensions (Section $10.2)$.

4.2.6. sBIT Significant bits

To simplify decoders, PNG specifies that only certain sample depths can be used, and further specifies that sample values should be scaled to the full range of possible values at the sample depth. However, the sBIT chunk is provided in order to store the original number of significant bits. This allows 
decoders to recover the original data losslessly even if the data had a sample depth not directly supported by PNG. We recommend that an encoder emit an sBIT chunk if it has converted the data from a lower sample depth.

For color type 0 (grayscale), the sBIT chunk contains a single byte, indicating the number of bits that were significant in the source data.

For color type 2 (truecolor), the sBIT chunk contains three bytes, indicating the number of bits that were significant in the source data for the red, green, and blue channels, respectively.

For color type 3 (indexed color), the sBIT chunk contains three bytes, indicating the number of bits that were significant in the source data for the red, green, and blue components of the palette entries, respectively.

For color type 4 (grayscale with alpha channel), the sBIT chunk contains two bytes, indicating the number of bits that were significant in the source grayscale data and the source alpha data, respectively.

For color type 6 (truecolor with alpha channel), the sBIT chunk contains four bytes, indicating the number of bits that were significant in the source data for the red, green, blue and alpha channels, respectively.

Each depth specified in sBIT must be greater than zero and less than or equal to the sample depth (which is 8 for indexed-color images, and the bit depth given in IHDR for other color types).

A decoder need not pay attention to sBIT: the stored image is a valid PNG file of the sample depth indicated by IHDR. However, if the decoder wishes to recover the original data at its original precision, this can be done by right-shifting the stored samples (the stored palette entries, for an indexedcolor image). The encoder must scale the data in such a way that the high-order bits match the original data.

If the sBIT chunk appears, it must precede the first IDAT chunk, and it must also precede the PLTE chunk if present.

See Recommendations for Encoders: Sample depth scaling (Section 9.1) and Recommendations for Decoders: Sample depth rescaling (Section 10.4). 


\subsection{7. text Textual data}

Textual information that the encoder wishes to record with the image can be stored in tExt chunks. Each tExt chunk contains a keyword and a text string, in the format:

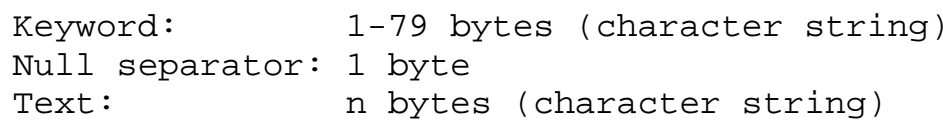

The keyword and text string are separated by a zero byte (null character). Neither the keyword nor the text string can contain a null character. Note that the text string is not null-terminated (the length of the chunk is sufficient information to locate the ending). The keyword must be at least one character and less than 80 characters long. The text string can be of any length from zero bytes up to the maximum permissible chunk size less the length of the keyword and separator.

Any number of text chunks can appear, and more than one with the same keyword is permissible.

The keyword indicates the type of information represented by the text string. The following keywords are predefined and should be used where appropriate:

$\begin{array}{ll}\text { Title } & \text { Short (one line) title or caption for image } \\ \text { Author } & \text { Name of image's creator } \\ \text { Description } & \text { Description of image (possibly long) } \\ \text { Copyright } & \text { Copyright notice } \\ \text { Creation Time } & \text { Time of original image creation } \\ \text { Software } & \text { Software used to create the image } \\ \text { Disclaimer } & \text { Legal disclaimer } \\ \text { Warning } & \text { Warning of nature of content } \\ \text { Source } & \text { Device used to create the image } \\ \text { Comment } & \text { Miscellaneous comment; conversion from } \\ & \text { GIF comment }\end{array}$

For the Creation Time keyword, the date format defined in section 5.2.14 of RFC 1123 is suggested, but not required [RFC-1123]. Decoders should allow for free-format text associated with this or any other keyword.

Other keywords may be invented for other purposes. Keywords of general interest can be registered with the maintainers of the PNG specification. However, it is also permitted to use private unregistered keywords. (Private keywords should be 
reasonably self-explanatory, in order to minimize the chance that the same keyword will be used for incompatible purposes by different people.)

Both keyword and text are interpreted according to the ISO 8859-1 (Latin-1) character set [ISO-8859]. The text string can contain any Latin-1 character. Newlines in the text string should be represented by a single linefeed character (decimal 10); use of other control characters in the text is discouraged.

Keywords must contain only printable Latin-1 characters and spaces; that is, only character codes 32-126 and 161-255 decimal are allowed. To reduce the chances for human misreading of a keyword, leading and trailing spaces are forbidden, as are consecutive spaces. Note also that the nonbreaking space (code 160) is not permitted in keywords, since it is visually indistinguishable from an ordinary space.

Keywords must be spelled exactly as registered, so that decoders can use simple literal comparisons when looking for particular keywords. In particular, keywords are considered case-sensitive.

See Recommendations for Encoders: Text chunk processing (Section 9.7) and Recommendations for Decoders: Text chunk processing (Section 10.11).

4.2.8. tIME Image last-modification time

The tIME chunk gives the time of the last image modification (not the time of initial image creation). It contains:

Year: 2 bytes (complete; for example, 1995, not 95)

Month: 1 byte $(1-12)$

Day: 1 byte $(1-31)$

Hour: 1 byte $(0-23)$

Minute: 1 byte $(0-59)$

Second: 1 byte $(0-60)$

(yes, 60, for leap seconds; not 61, a common error)

Universal Time (UTC, also called GMT) should be specified rather than local time. 
The tIME chunk is intended for use as an automatically-applied time stamp that is updated whenever the image data is changed. It is recommended that tIME not be changed by PNG editors that do not change the image data. See also the Creation Time tExt keyword, which can be used for a user-supplied time.

\subsection{9. tRNS Transparency}

The tRNS chunk specifies that the image uses simple transparency: either alpha values associated with palette entries (for indexed-color images) or a single transparent color (for grayscale and truecolor images). Although simple transparency is not as elegant as the full alpha channel, it requires less storage space and is sufficient for many common cases.

For color type 3 (indexed color), the tRNS chunk contains a series of one-byte alpha values, corresponding to entries in the PLTE chunk:

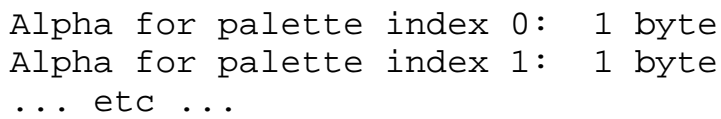

Each entry indicates that pixels of the corresponding palette index must be treated as having the specified alpha value. Alpha values have the same interpretation as in an 8-bit full alpha channel: 0 is fully transparent, 255 is fully opaque, regardless of image bit depth. The tRNS chunk must not contain more alpha values than there are palette entries, but tRNS can contain fewer values than there are palette entries. In this case, the alpha value for all remaining palette entries is assumed to be 255. In the common case in which only palette index 0 need be made transparent, only a one-byte tRNS chunk is needed.

For color type 0 (grayscale), the tRNS chunk contains a single gray level value, stored in the format:

Gray: 2 bytes, range $0 \ldots\left(2^{\wedge}\right.$ bitdepth $)-1$

(For consistency, 2 bytes are used regardless of the image bit depth.) Pixels of the specified gray level are to be treated as transparent (equivalent to alpha value 0); all other pixels are to be treated as fully opaque (alpha value (2^bitdepth)-1). 
For color type 2 (truecolor), the tRNS chunk contains a single RGB color value, stored in the format:

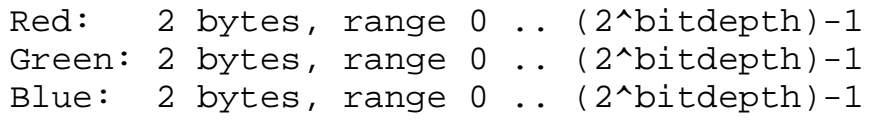

(For consistency, 2 bytes per sample are used regardless of the image bit depth.) Pixels of the specified color value are to be treated as transparent (equivalent to alpha value 0); all other pixels are to be treated as fully opaque (alpha value $\left(2^{\wedge}\right.$ bitdepth $\left.)-1\right)$.

tRNS is prohibited for color types 4 and 6 , since a full alpha channel is already present in those cases.

Note: when dealing with 16-bit grayscale or truecolor data, it is important to compare both bytes of the sample values to determine whether a pixel is transparent. Although decoders may drop the low-order byte of the samples for display, this must not occur until after the data has been tested for transparency. For example, if the grayscale level 0x0001 is specified to be transparent, it would be incorrect to compare only the high-order byte and decide that 0x0002 is also transparent.

When present, the tRNS chunk must precede the first IDAT chunk, and must follow the PLTE chunk, if any.

\subsubsection{0. zTXt Compressed textual data}

The zTXt chunk contains textual data, just as tExt does; however, zTXt takes advantage of compression. zTXt and tEXt chunks are semantically equivalent, but zTXt is recommended for storing large blocks of text.

A zTXt chunk contains:

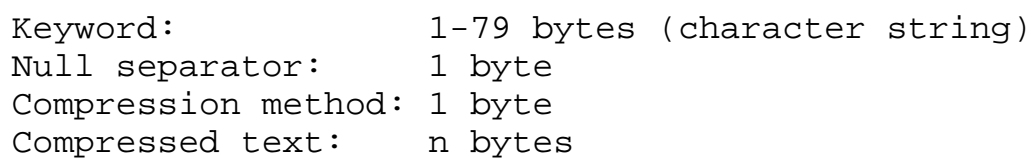

The keyword and null separator are exactly the same as in the tExt chunk. Note that the keyword is not compressed. The compression method byte identifies the compression method used in this zTXt chunk. The only value presently defined for it is 0 (deflate/inflate compression). The compression method byte is 
followed by a compressed datastream that makes up the remainder of the chunk. For compression method 0, this datastream adheres to the zlib datastream format (see Deflate/Inflate Compression, Chapter 5). Decompression of this datastream yields Latin-1 text that is identical to the text that would be stored in an equivalent tExt chunk.

Any number of zTXt and tExt chunks can appear in the same file. See the preceding definition of the tExt chunk for the predefined keywords and the recommended format of the text.

See Recommendations for Encoders: Text chunk processing (Section 9.7), and Recommendations for Decoders: Text chunk processing (Section 10.11).

4.3. Summary of standard chunks

This table summarizes some properties of the standard chunk types.

Critical chunks (must appear in this order, except PLTE

is optional):

Name Multiple Ordering constraints OK?

IHDR No Must be first

PLTE No Before IDAT

IDAT Yes Multiple IDATs must be consecutive

IEND No Must be last

Ancillary chunks (need not appear in this order):

$\begin{array}{lll}\text { Name } & \begin{array}{c}\text { Multiple } \\ \text { OK? }\end{array} & \text { Ordering constraints } \\ \text { cHRM } & \text { No } & \text { Before PLTE and IDAT } \\ \text { gAMA } & \text { No } & \text { Before PLTE and IDAT } \\ \text { SBIT } & \text { No } & \text { Before PLTE and IDAT } \\ \text { bKGD } & \text { No } & \text { After PLTE; before IDAT } \\ \text { hIST } & \text { No } & \text { After PLTE; before IDAT } \\ \text { tRNS } & \text { No } & \text { After PLTE; before IDAT } \\ \text { pHYs } & \text { No } & \text { Before IDAT } \\ \text { tIME } & \text { No } & \text { None } \\ \text { tEXt } & \text { Yes } & \text { None } \\ \text { zTXt } & \text { Yes } & \text { None }\end{array}$


Standard keywords for tExt and zTXt chunks:

$\begin{array}{ll}\text { Title } & \text { Short (one line) title or caption for image } \\ \text { Author } & \text { Name of image's creator } \\ \text { Description } & \text { Description of image (possibly long) } \\ \text { Copyright } & \text { Copyright notice } \\ \text { Creation Time } & \text { Time of original image creation } \\ \text { Software } & \text { Software used to create the image } \\ \text { Disclaimer } & \text { Legal disclaimer } \\ \text { Warning } & \text { Warning of nature of content } \\ \text { Source } & \text { Device used to create the image } \\ \text { Comment } & \text { Miscellaneous comment; conversion from } \\ & \text { GIF comment }\end{array}$

4.4. Additional chunk types

Additional public PNG chunk types are defined in the document "PNG Special-Purpose Public Chunks" [PNG-EXTENSIONS]. Chunks described there are expected to be less widely supported than those defined in this specification. However, application authors are encouraged to use those chunk types whenever appropriate for their applications. Additional chunk types can be proposed for inclusion in that list by contacting the PNG specification maintainers at png-info@uunet.uu.net or at png-group@w3.org.

New public chunks will only be registered if they are of use to others and do not violate the design philosophy of PNG. Chunk registration is not automatic, although it is the intent of the authors that it be straightforward when a new chunk of potentially wide application is needed. Note that the creation of new critical chunk types is discouraged unless absolutely necessary.

Applications can also use private chunk types to carry data that is not of interest to other applications. See Recommendations for Encoders: Use of private chunks (Section 9.8).

Decoders must be prepared to encounter unrecognized public or private chunk type codes. Unrecognized chunk types must be handled as described in Chunk naming conventions (Section 3.3).

5. Deflate/Inflate Compression

PNG compression method 0 (the only compression method presently defined for PNG) specifies deflate/inflate compression with a $32 \mathrm{~K}$ sliding window. Deflate compression is an Lz77 derivative used in zip, gzip, pkzip and related programs. Extensive research has been done supporting its patent-free status. Portable C implementations are freely available. 


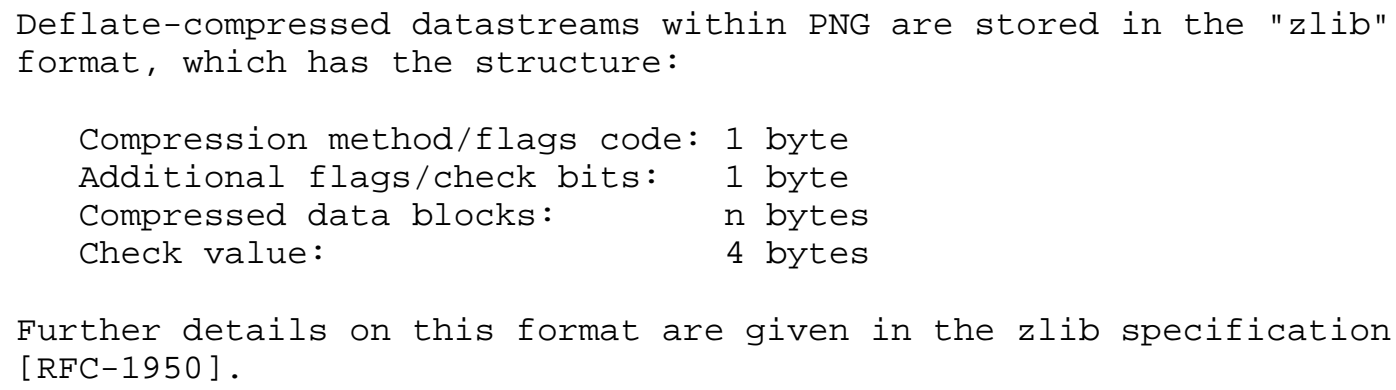

For PNG compression method 0, the zlib compression method/flags code must specify method code 8 ("deflate" compression) and an Lz77 window size of not more than $32 \mathrm{~K}$. Note that the zlib compression method number is not the same as the PNG compression method number. The additional flags must not specify a preset dictionary.

The compressed data within the zlib datastream is stored as a series of blocks, each of which can represent raw (uncompressed) data, LZ77-compressed data encoded with fixed Huffman codes, or Lz77compressed data encoded with custom Huffman codes. A marker bit in the final block identifies it as the last block, allowing the decoder to recognize the end of the compressed datastream. Further details on the compression algorithm and the encoding are given in the deflate specification [RFC-1951].

The check value stored at the end of the zlib datastream is calculated on the uncompressed data represented by the datastream. Note that the algorithm used is not the same as the CRC calculation used for PNG chunk check values. The zlib check value is useful mainly as a cross-check that the deflate and inflate algorithms are implemented correctly. Verifying the chunk CRCs provides adequate confidence that the PNG file has been transmitted undamaged.

In a PNG file, the concatenation of the contents of all the IDAT chunks makes up a zlib datastream as specified above. This datastream decompresses to filtered image data as described elsewhere in this document.

It is important to emphasize that the boundaries between IDAT chunks are arbitrary and can fall anywhere in the zlib datastream. There is not necessarily any correlation between IDAT chunk boundaries and deflate block boundaries or any other feature of the zlib data. For example, it is entirely possible for the terminating zlib check value to be split across IDAT chunks. 
In the same vein, there is no required correlation between the structure of the image data (i.e., scanline boundaries) and deflate block boundaries or IDAT chunk boundaries. The complete image data is represented by a single zlib datastream that is stored in some number of IDAT chunks; a decoder that assumes any more than this is incorrect. (Of course, some encoder implementations may emit files in which some of these structures are indeed related. But decoders cannot rely on this.)

PNG also uses zlib datastreams in zTXt chunks. In a zTXt chunk, the remainder of the chunk following the compression method byte is a zlib datastream as specified above. This datastream decompresses to the user-readable text described by the chunk's keyword. Unlike the image data, such datastreams are not split across chunks; each zTXt chunk contains an independent zlib datastream.

Additional documentation and portable $\mathrm{C}$ code for deflate and inflate are available from the Info-zIP archives at

<URL: ftp://ftp.uu.net/pub/archiving/zip/>.

6. Filter Algorithms

This chapter describes the filter algorithms that can be applied before compression. The purpose of these filters is to prepare the image data for optimum compression.

\subsection{Filter types}

PNG filter method 0 defines five basic filter types:

$\begin{array}{ll}\text { Type } & \text { Name } \\ 0 & \text { None } \\ 1 & \text { Sub } \\ 2 & \text { Up } \\ 3 & \text { Average } \\ 4 & \text { Paeth }\end{array}$

(Note that filter method 0 in IHDR specifies exactly this set of five filter types. If the set of filter types is ever extended, a different filter method number will be assigned to the extended set, so that decoders need not decompress the data to discover that it contains unsupported filter types.)

The encoder can choose which of these filter algorithms to apply on a scanline-by-scanline basis. In the image data sent to the compression step, each scanline is preceded by a filter type byte that specifies the filter algorithm used for that scanline. 
Filtering algorithms are applied to bytes, not to pixels, regardless of the bit depth or color type of the image. The filtering algorithms work on the byte sequence formed by a scanline that has been represented as described in Image layout (Section 2.3). If the image includes an alpha channel, the alpha data is filtered in the same way as the image data.

When the image is interlaced, each pass of the interlace pattern is treated as an independent image for filtering purposes. The filters work on the byte sequences formed by the pixels actually transmitted during a pass, and the "previous scanline" is the one previously transmitted in the same pass, not the one adjacent in the complete image. Note that the subimage transmitted in any one pass is always rectangular, but is of smaller width and/or height than the complete image. Filtering is not applied when this subimage is empty.

For all filters, the bytes "to the left of" the first pixel in a scanline must be treated as being zero. For filters that refer to the prior scanline, the entire prior scanline must be treated as being zeroes for the first scanline of an image (or of a pass of an interlaced image).

To reverse the effect of a filter, the decoder must use the decoded values of the prior pixel on the same line, the pixel immediately above the current pixel on the prior line, and the pixel just to the left of the pixel above. This implies that at least one scanline's worth of image data will have to be stored by the decoder at all times. Even though some filter types do not refer to the prior scanline, the decoder will always need to store each scanline as it is decoded, since the next scanline might use a filter that refers to it.

PNG imposes no restriction on which filter types can be applied to an image. However, the filters are not equally effective on all types of data. See Recommendations for Encoders: Filter selection (Section 9.6).

See also Rationale: Filtering (Section 12.9).

\subsection{Filter type 0: None}

With the None filter, the scanline is transmitted unmodified; it is only necessary to insert a filter type byte before the data. 


\subsection{Filter type 1: Sub}

The Sub filter transmits the difference between each byte and the value of the corresponding byte of the prior pixel.

To compute the sub filter, apply the following formula to each byte of the scanline:

$$
\operatorname{Sub}(x)=\operatorname{Raw}(x)-\operatorname{Raw}(x-b p p)
$$

where $\mathrm{x}$ ranges from zero to the number of bytes representing the scanline minus one, Raw $(x)$ refers to the raw data byte at that byte position in the scanline, and bpp is defined as the number of bytes per complete pixel, rounding up to one. For example, for color type 2 with a bit depth of 16 , bpp is equal to 6 (three samples, two bytes per sample); for color type 0 with a bit depth of 2, bpp is equal to 1 (rounding up); for color type 4 with a bit depth of 16 , bpp is equal to 4 (two-byte grayscale sample, plus two-byte alpha sample).

Note this computation is done for each byte, regardless of bit depth. In a 16-bit image, each MSB is predicted from the preceding MSB and each LSB from the preceding LSB, because of the way that bpp is defined.

Unsigned arithmetic modulo 256 is used, so that both the inputs and outputs fit into bytes. The sequence of sub values is transmitted as the filtered scanline.

For all $\mathrm{x}<0$, assume $\operatorname{Raw}(\mathrm{x})=0$.

To reverse the effect of the sub filter after decompression, output the following value:

$$
\operatorname{Sub}(x)+\operatorname{Raw}(x-b p p)
$$

(computed mod 256), where Raw refers to the bytes already decoded.

\subsection{Filter type 2: Up}

The Up filter is just like the sub filter except that the pixel immediately above the current pixel, rather than just to its left, is used as the predictor.

To compute the Up filter, apply the following formula to each byte of the scanline:

$$
\operatorname{Up}(x)=\operatorname{Raw}(x)-\operatorname{Prior}(x)
$$


where $x$ ranges from zero to the number of bytes representing the scanline minus one, Raw $(x)$ refers to the raw data byte at that byte position in the scanline, and Prior(x) refers to the unfiltered bytes of the prior scanline.

Note this is done for each byte, regardless of bit depth. Unsigned arithmetic modulo 256 is used, so that both the inputs and outputs fit into bytes. The sequence of Up values is transmitted as the filtered scanline.

On the first scanline of an image (or of a pass of an interlaced image), assume Prior $(\mathrm{x})=0$ for all $\mathrm{x}$.

To reverse the effect of the Up filter after decompression, output the following value:

$$
\mathrm{Up}(\mathrm{x})+\operatorname{Prior}(\mathrm{x})
$$

(computed mod 256), where Prior refers to the decoded bytes of the prior scanline.

\subsection{Filter type 3: Average}

The Average filter uses the average of the two neighboring pixels (left and above) to predict the value of a pixel.

To compute the Average filter, apply the following formula to each byte of the scanline:

$$
\text { Average }(x)=\operatorname{Raw}(x)-f l o o r((\operatorname{Raw}(x-b p p)+\operatorname{Prior}(x)) / 2)
$$

where $\mathrm{x}$ ranges from zero to the number of bytes representing the scanline minus one, Raw $(x)$ refers to the raw data byte at that byte position in the scanline, Prior(x) refers to the unfiltered bytes of the prior scanline, and bpp is defined as for the sub filter.

Note this is done for each byte, regardless of bit depth. The sequence of Average values is transmitted as the filtered scanline.

The subtraction of the predicted value from the raw byte must be done modulo 256, so that both the inputs and outputs fit into bytes. However, the sum Raw (x-bpp)+Prior (x) must be formed without overflow (using at least nine-bit arithmetic). floor() indicates that the result of the division is rounded to the next lower integer if fractional; in other words, it is an integer division or right shift operation. 
For all $\mathrm{x}<0$, assume Raw $(\mathrm{x})=0$. On the first scanline of an image (or of a pass of an interlaced image), assume Prior $(x)=0$ for all $\mathrm{x}$.

To reverse the effect of the Average filter after decompression, output the following value:

$$
\text { Average }(x)+f l o o r((\operatorname{Raw}(x-b p p)+\operatorname{Prior}(x)) / 2)
$$

where the result is computed mod 256, but the prediction is calculated in the same way as for encoding. Raw refers to the bytes already decoded, and Prior refers to the decoded bytes of the prior scanline.

6.6. Filter type 4: Paeth

The Paeth filter computes a simple linear function of the three neighboring pixels (left, above, upper left), then chooses as predictor the neighboring pixel closest to the computed value. This technique is due to Alan W. Paeth [PAETH].

To compute the Paeth filter, apply the following formula to each byte of the scanline:

$$
\begin{gathered}
\operatorname{Paeth}(\mathrm{x})=\operatorname{Raw}(\mathrm{x})-\operatorname{PaethPredictor}(\operatorname{Raw}(\mathrm{x}-\mathrm{bpp}), \operatorname{Prior}(\mathrm{x}), \\
\operatorname{Prior}(\mathrm{x}-\mathrm{bpp}))
\end{gathered}
$$

where $\mathrm{x}$ ranges from zero to the number of bytes representing the scanline minus one, Raw $(x)$ refers to the raw data byte at that byte position in the scanline, Prior(x) refers to the unfiltered bytes of the prior scanline, and bpp is defined as for the sub filter.

Note this is done for each byte, regardless of bit depth. Unsigned arithmetic modulo 256 is used, so that both the inputs and outputs fit into bytes. The sequence of Paeth values is transmitted as the filtered scanline. 


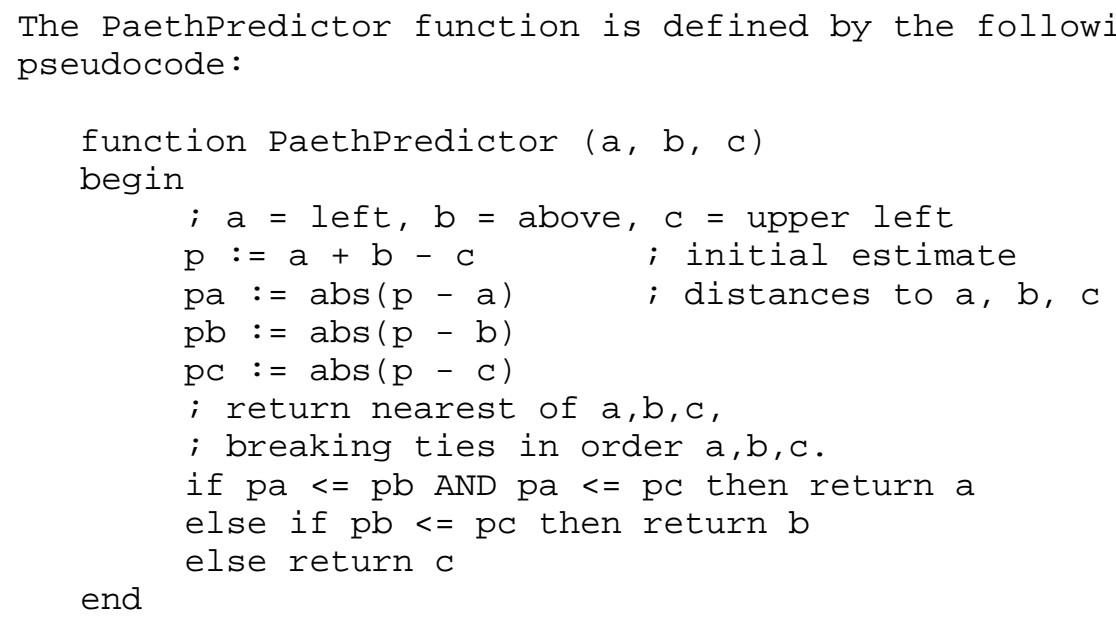

The calculations within the PaethPredictor function must be performed exactly, without overflow. Arithmetic modulo 256 is to be used only for the final step of subtracting the function result from the target byte value.

Note that the order in which ties are broken is critical and must not be altered. The tie break order is: pixel to the left, pixel above, pixel to the upper left. (This order differs from that given in Paeth's article.)

For all $\mathrm{x}<0$, assume Raw $(\mathrm{x})=0$ and Prior $(\mathrm{x})=0$. On the first scanline of an image (or of a pass of an interlaced image), assume Prior $(\mathrm{x})=0$ for all $\mathrm{x}$.

To reverse the effect of the Paeth filter after decompression, output the following value:

Paeth $(x)+$ PaethPredictor (Raw (x-bpp), Prior (x), Prior (x-bpp))

(computed mod 256), where Raw and Prior refer to bytes already decoded. Exactly the same PaethPredictor function is used by both encoder and decoder.

7. Chunk Ordering Rules

To allow new chunk types to be added to PNG, it is necessary to establish rules about the ordering requirements for all chunk types. Otherwise a PNG editing program cannot know what to do when it encounters an unknown chunk. 
We define a "PNG editor" as a program that modifies a PNG file and wishes to preserve as much as possible of the ancillary information in the file. Two examples of PNG editors are a program that adds or modifies text chunks, and a program that adds a suggested palette to a truecolor PNG file. Ordinary image editors are not PNG editors in this sense, because they usually discard all unrecognized information while reading in an image. (Note: we strongly encourage programs handling PNG files to preserve ancillary information whenever possible.)

As an example of possible problems, consider a hypothetical new ancillary chunk type that is safe-to-copy and is required to appear after PLTE if PLTE is present. If our program to add a suggested PLTE does not recognize this new chunk, it may insert PLTE in the wrong place, namely after the new chunk. We could prevent such problems by requiring PNG editors to discard all unknown chunks, but that is a very unattractive solution. Instead, PNG requires ancillary chunks not to have ordering restrictions like this.

To prevent this type of problem while allowing for future extension, we put some constraints on both the behavior of PNG editors and the allowed ordering requirements for chunks.

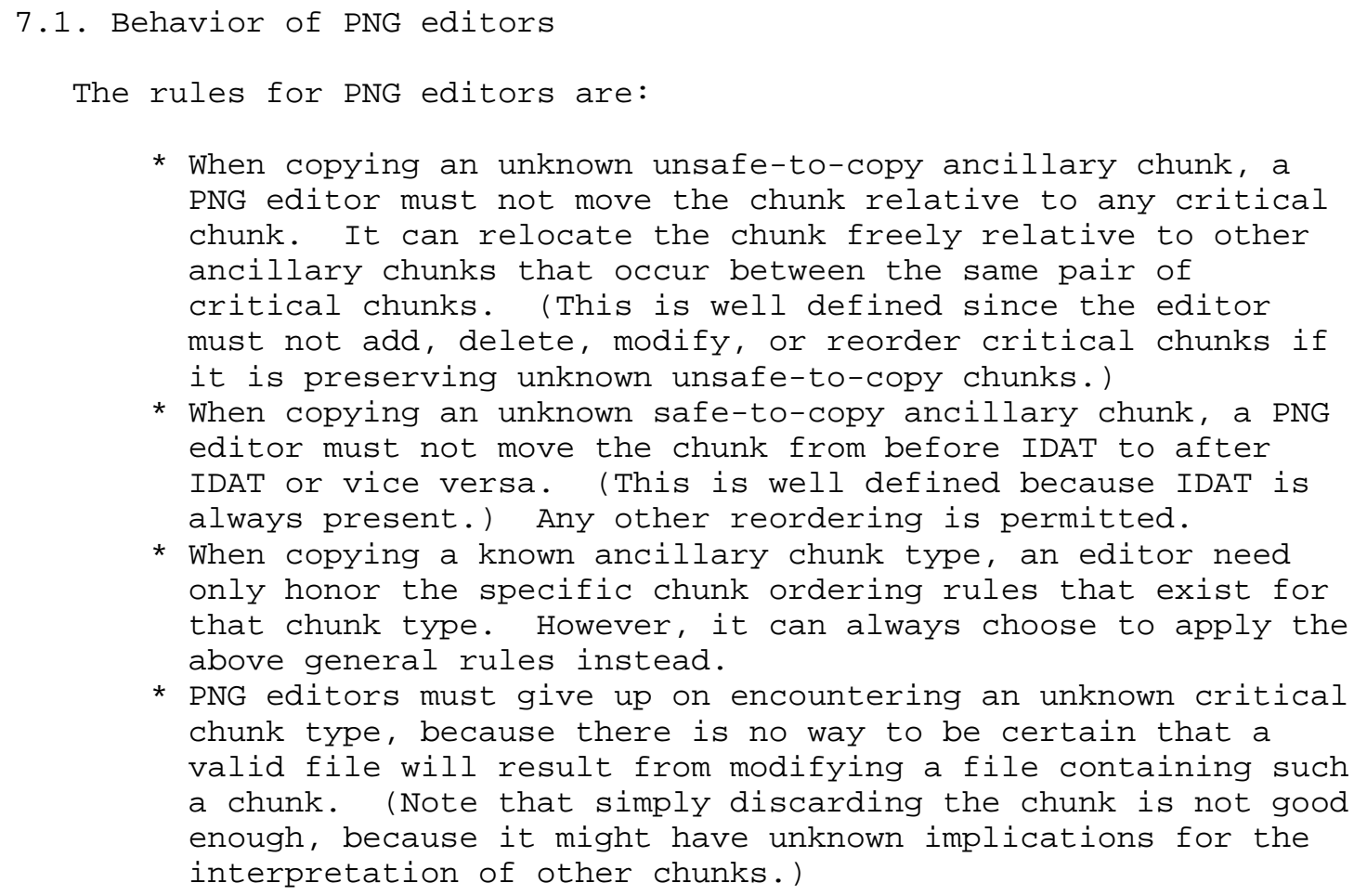


These rules are expressed in terms of copying chunks from an input file to an output file, but they apply in the obvious way if a PNG file is modified in place.

See also Chunk naming conventions (Section 3.3).

7.2. Ordering of ancillary chunks

The ordering rules for an ancillary chunk type cannot be any stricter than this:

* Unsafe-to-copy chunks can have ordering requirements relative to critical chunks.

* Safe-to-copy chunks can have ordering requirements relative to IDAT.

The actual ordering rules for any particular ancillary chunk type may be weaker. See for example the ordering rules for the standard ancillary chunk types (Summary of standard chunks, Section 4.3).

Decoders must not assume more about the positioning of any ancillary chunk than is specified by the chunk ordering rules. In particular, it is never valid to assume that a specific ancillary chunk type occurs with any particular positioning relative to other ancillary chunks. (For example, it is unsafe to assume that your private ancillary chunk occurs immediately before IEND. Even if your application always writes it there, a PNG editor might have inserted some other ancillary chunk after it. But you can safely assume that your chunk will remain somewhere between IDAT and IEND.)

\subsection{Ordering of critical chunks}

Critical chunks can have arbitrary ordering requirements, because PNG editors are required to give up if they encounter unknown critical chunks. For example, IHDR has the special ordering rule that it must always appear first. A PNG editor, or indeed any PNG-writing program, must know and follow the ordering rules for any critical chunk type that it can emit. 
8. Miscellaneous Topics

8.1. File name extension

On systems where file names customarily include an extension signifying file type, the extension ".png" is recommended for PNG files. Lower case ".png" is preferred if file names are casesensitive.

8.2. Internet media type

The Internet Assigned Numbers Authority (IANA) has registered "image/png" as the Internet Media Type for PNG [RFC-2045, RFC2048]. For robustness, decoders may choose to also support the interim media type "image/x-png" which was in use before registration was complete.

8.3. Macintosh file layout

In the Apple Macintosh system, the following conventions are recommended:

* The four-byte file type code for PNG files is "PNGf". (This code has been registered with Apple for PNG files.) The creator code will vary depending on the creating application.

* The contents of the data fork must be a PNG file exactly as described in the rest of this specification.

* The contents of the resource fork are unspecified. It may be empty or may contain application-dependent resources.

* When transferring a Macintosh PNG file to a non-Macintosh system, only the data fork should be transferred.

\subsection{Multiple-image extension}

PNG itself is strictly a single-image format. However, it may be necessary to store multiple images within one file; for example, this is needed to convert some GIF files. In the future, a multiple-image format based on PNG may be defined. Such a format will be considered a separate file format and will have a different signature. PNG-supporting applications may or may not choose to support the multiple-image format.

See Rationale: Why not these features? (Section 12.3). 


\subsection{Security considerations}

A PNG file or datastream is composed of a collection of explicitly typed "chunks". Chunks whose contents are defined by the specification could actually contain anything, including malicious code. But there is no known risk that such malicious code could be executed on the recipient's computer as a result of decoding the PNG image.

The possible security risks associated with future chunk types cannot be specified at this time. Security issues will be considered when evaluating chunks proposed for registration as public chunks. There is no additional security risk associated with unknown or unimplemented chunk types, because such chunks will be ignored, or at most be copied into another PNG file.

The tExt and zTXt chunks contain data that is meant to be displayed as plain text. It is possible that if the decoder displays such text without filtering out control characters, especially the ESC (escape) character, certain systems or terminals could behave in undesirable and insecure ways. We recommend that decoders filter out control characters to avoid this risk; see Recommendations for Decoders: Text chunk processing (Section 10.11).

Because every chunk's length is available at its beginning, and because every chunk has a CRC trailer, there is a very robust defense against corrupted data and against fraudulent chunks that attempt to overflow the decoder's buffers. Also, the PNG signature bytes provide early detection of common file transmission errors.

A decoder that fails to check CRCs could be subject to data corruption. The only likely consequence of such corruption is incorrectly displayed pixels within the image. Worse things might happen if the CRC of the IHDR chunk is not checked and the width or height fields are corrupted. See Recommendations for Decoders: Error checking (Section 10.1).

A poorly written decoder might be subject to buffer overflow, because chunks can be extremely large, up to (2^31)-1 bytes long. But properly written decoders will handle large chunks without difficulty. 


\section{Recommendations for Encoders}

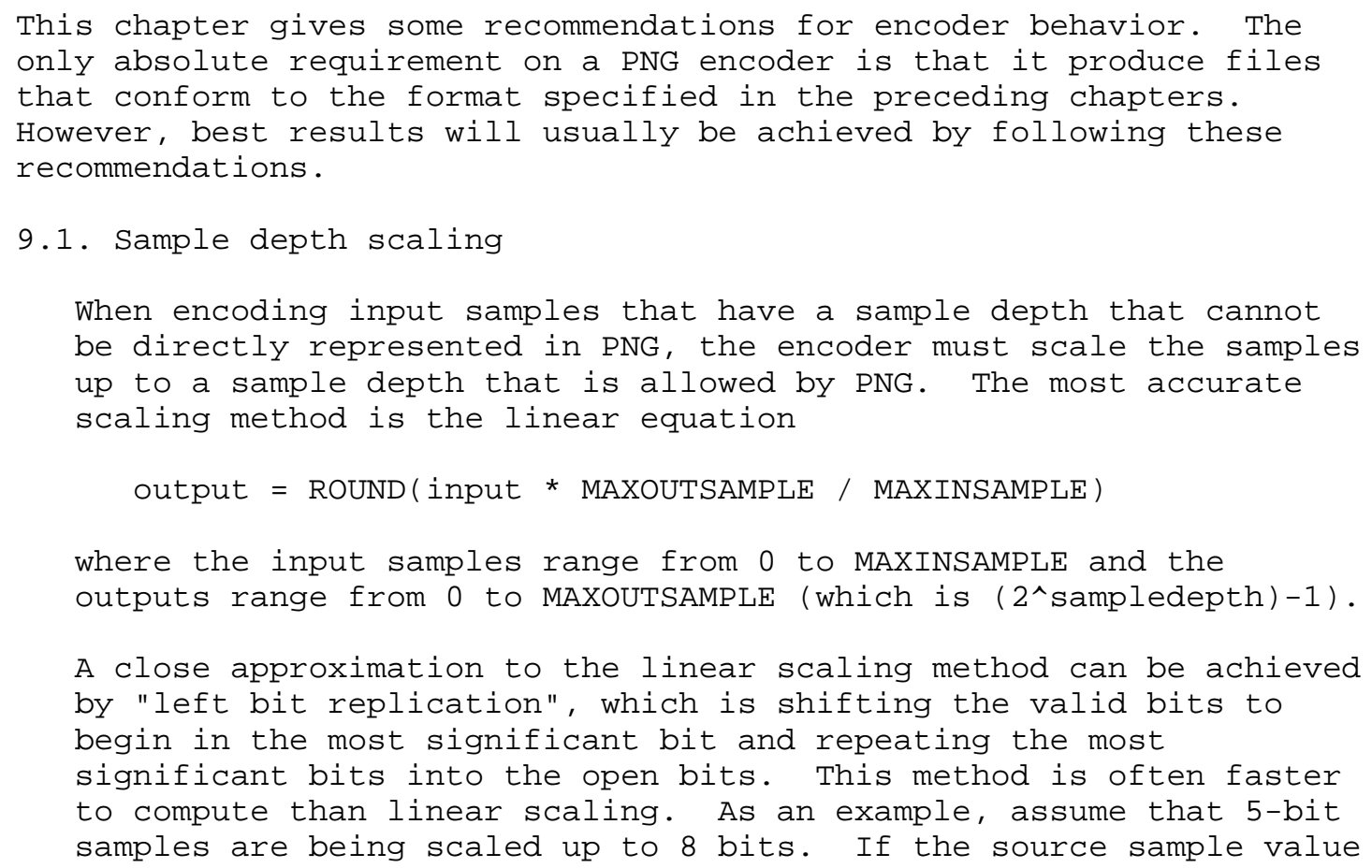


A distinctly less accurate approximation is obtained by simply left-shifting the input value and filling the low order bits with zeroes. This scheme cannot reproduce white exactly, since it does not generate an all-ones maximum value; the net effect is to darken the image slightly. This method is not recommended in general, but it does have the effect of improving compression, particularly when dealing with greater-than-eight-bit sample depths. Since the relative error introduced by zero-fill scaling is small at high sample depths, some encoders may choose to use it. Zero-fill must not be used for alpha channel data, however, since many decoders will special-case alpha values of all zeroes and all ones. It is important to represent both those values exactly in the scaled data.

When the encoder writes an sBIT chunk, it is required to do the scaling in such a way that the high-order bits of the stored samples match the original data. That is, if the sBIT chunk specifies a sample depth of $\mathrm{S}$, the high-order $\mathrm{S}$ bits of the stored data must agree with the original s-bit data values. This allows decoders to recover the original data by shifting right. The added low-order bits are not constrained. Note that all the above scaling methods meet this restriction.

When scaling up source data, it is recommended that the low-order bits be filled consistently for all samples; that is, the same source value should generate the same sample value at any pixel position. This improves compression by reducing the number of distinct sample values. However, this is not a requirement, and some encoders may choose not to follow it. For example, an encoder might instead dither the low-order bits, improving displayed image quality at the price of increasing file size.

In some applications the original source data may have a range that is not a power of 2. The linear scaling equation still works for this case, although the shifting methods do not. It is recommended that an SBIT chunk not be written for such images, since sBIT suggests that the original data range was exactly $0.2^{\wedge} \mathrm{S}-1$.

\subsection{Encoder gamma handling}

See Gamma Tutorial (Chapter 13) if you aren't already familiar with gamma issues.

Proper handling of gamma encoding and the gAMA chunk in an encoder depends on the prior history of the sample values and on whether these values have already been quantized to integers. 
If the encoder has access to sample intensity values in floatingpoint or high-precision integer form (perhaps from a computer image renderer), then it is recommended that the encoder perform its own gamma encoding before quantizing the data to integer values for storage in the file. Applying gamma encoding at this stage results in images with fewer banding artifacts at a given sample depth, or allows smaller samples while retaining the same visual quality.

A linear intensity level, expressed as a floating-point value in the range 0 to 1 , can be converted to a gamma-encoded sample value by

$$
\text { sample }=\text { ROUND ( (intensity ^ encoder_gamma) * MAXSAMPLE) }
$$

The file_gamma value to be written in the PNG gAMA chunk is the same as encoder_gamma in this equation, since we are assuming the initial intensity value is linear (in effect, camera_gamma is $1.0)$.

If the image is being written to a file only, the encoder_gamma value can be selected somewhat arbitrarily. Values of 0.45 or 0.5 are generally good choices because they are common in video systems, and so most PNG decoders should do a good job displaying such images.

Some image renderers may simultaneously write the image to a PNG file and display it on-screen. The displayed pixels should be gamma corrected for the display system and viewing conditions in use, so that the user sees a proper representation of the intended scene. An appropriate gamma correction value is

$$
\text { screen_gc = viewing_gamma / display_gamma }
$$

If the renderer wants to write the same gamma-corrected sample values to the PNG file, avoiding a separate gamma-encoding step for file output, then this screen_gc value should be written in the gAMA chunk. This will allow a PNG decoder to reproduce what the file's originator saw on screen during rendering (provided the decoder properly supports arbitrary values in a gAMA chunk).

However, it is equally reasonable for a renderer to apply gamma correction for screen display using a gamma appropriate to the viewing conditions, and to separately gamma-encode the sample values for file storage using a standard value of gamma such as 0.5. In fact, this is preferable, since some PNG decoders may not accurately display images with unusual gAMA values. 
Computer graphics renderers often do not perform gamma encoding, instead making sample values directly proportional to scene light intensity. If the PNG encoder receives sample values that have already been quantized into linear-light integer values, there is no point in doing gamma encoding on them; that would just result in further loss of information. The encoder should just write the sample values to the PNG file. This "linear" sample encoding is equivalent to gamma encoding with a gamma of 1.0, so graphics programs that produce linear samples should always emit a gAMA chunk specifying a gamma of 1.0 .

When the sample values come directly from a piece of hardware, the correct gAMA value is determined by the gamma characteristic of the hardware. In the case of video digitizers ("frame grabbers"), gAMA should be 0.45 or 0.5 for NTSC (possibly less for PAL or SECAM) since video camera transfer functions are standardized. Image scanners are less predictable. Their output samples may be linear (gamma 1.0) since CCD sensors themselves are linear, or the scanner hardware may have already applied gamma correction designed to compensate for dot gain in subsequent printing (gamma of about 0.57), or the scanner may have corrected the samples for display on a CRT (gamma of 0.4-0.5). You will need to refer to the scanner's manual, or even scan a calibrated gray wedge, to determine what a particular scanner does.

File format converters generally should not attempt to convert supplied images to a different gamma. Store the data in the PNG file without conversion, and record the source gamma if it is known. Gamma alteration at file conversion time causes requantization of the set of intensity levels that are represented, introducing further roundoff error with little benefit. It's almost always better to just copy the sample values intact from the input to the output file.

In some cases, the supplied image may be in an image format (e.g., TIFF) that can describe the gamma characteristic of the image. In such cases, a file format converter is strongly encouraged to write a PNG gAMA chunk that corresponds to the known gamma of the source image. Note that some file formats specify the gamma of the display system, not the camera. If the input file's gamma value is greater than 1.0 , it is almost certainly a display system gamma, and you should use its reciprocal for the PNG gAMA. 
If the encoder or file format converter does not know how an image was originally created, but does know that the image has been displayed satisfactorily on a display with gamma display_gamma under lighting conditions where a particular viewing_gamma is appropriate, then the image can be marked as having the file_gamma:

file_gamma = viewing_gamma / display_gamma

This will allow viewers of the PNG file to see the same image that the person running the file format converter saw. Although this may not be precisely the correct value of the image gamma, it's better to write a gAMA chunk with an approximately right value than to omit the chunk and force PNG decoders to guess at an appropriate gamma.

On the other hand, if the image file is being converted as part of a "bulk" conversion, with no one looking at each image, then it is better to omit the gAMA chunk entirely. If the image gamma has to be guessed at, leave it to the decoder to do the guessing.

Gamma does not apply to alpha samples; alpha is always represented linearly.

See also Recommendations for Decoders: Decoder gamma handling (Section 10.5).

9.3. Encoder color handling

See Color Tutorial (Chapter 14) if you aren't already familiar with color issues.

If it is possible for the encoder to determine the chromaticities of the source display primaries, or to make a strong guess based on the origin of the image or the hardware running it, then the encoder is strongly encouraged to output the CHRM chunk. If it does so, the gAMA chunk should also be written; decoders can do little with cHRM if gAMA is missing. 
Video created with recent video equipment probably uses the CCIR 709 primaries and D65 white point [ITU-BT709], which are:

$\begin{array}{ccccl} & \mathrm{R} & \mathrm{G} & \mathrm{B} & \text { White } \\ \mathrm{x} & 0.640 & 0.300 & 0.150 & 0.3127 \\ \mathrm{y} & 0.330 & 0.600 & 0.060 & 0.3290\end{array}$

An older but still very popular video standard is SMPTE-C [SMPTE$170 \mathrm{M}]$ :

$\begin{array}{ccccl} & \mathrm{R} & \mathrm{G} & \mathrm{B} & \text { White } \\ \mathrm{x} & 0.630 & 0.310 & 0.155 & 0.3127 \\ \mathrm{y} & 0.340 & 0.595 & 0.070 & 0.3290\end{array}$

The original NTSC color primaries have not been used in decades. Although you may still find the NTSC numbers listed in standards documents, you won't find any images that actually use them.

Scanners that produce PNG files as output should insert the filter chromaticities into a cHRM chunk and the camera_gamma into a gAMA chunk.

In the case of hand-drawn or digitally edited images, you have to determine what monitor they were viewed on when being produced. Many image editing programs allow you to specify what type of monitor you are using. This is often because they are working in some device-independent space internally. Such programs have enough information to write valid CHRM and gAMA chunks, and should do so automatically.

If the encoder is compiled as a portion of a computer image renderer that performs full-spectral rendering, the monitor values that were used to convert from the internal device-independent color space to RGB should be written into the cHRM chunk. Any colors that are outside the gamut of the chosen RGB device should be clipped or otherwise constrained to be within the gamut; PNG does not store out of gamut colors.

If the computer image renderer performs calculations directly in device-dependent RGB space, a cHRM chunk should not be written unless the scene description and rendering parameters have been adjusted to look good on a particular monitor. In that case, the data for that monitor (if known) should be used to construct a cHRM chunk. 
There are often cases where an image's exact origins are unknown, particularly if it began life in some other format. A few image formats store calibration information, which can be used to fill in the cHRM chunk. For example, all PhotoCD images use the CCIR 709 primaries and D65 whitepoint, so these values can be written into the CHRM chunk when converting a PhotoCD file. PhotoCD also uses the SMPTE-170M transfer function, which is closely approximated by a gAMA of 0.5. (PhotoCD can store colors outside the RGB gamut, so the image data will require gamut mapping before writing to PNG format.) TIFF 6.0 files can optionally store calibration information, which if present should be used to construct the cHRM chunk. GIF and most other formats do not store any calibration information.

It is not recommended that file format converters attempt to convert supplied images to a different RGB color space. Store the data in the PNG file without conversion, and record the source primary chromaticities if they are known. Color space transformation at file conversion time is a bad idea because of gamut mismatches and rounding errors. As with gamma conversions, it's better to store the data losslessly and incur at most one conversion when the image is finally displayed.

See also Recommendations for Decoders: Decoder color handling (Section 10.6).

\subsection{Alpha channel creation}

The alpha channel can be regarded either as a mask that temporarily hides transparent parts of the image, or as a means for constructing a non-rectangular image. In the first case, the color values of fully transparent pixels should be preserved for future use. In the second case, the transparent pixels carry no useful data and are simply there to fill out the rectangular image area required by PNG. In this case, fully transparent pixels should all be assigned the same color value for best compression.

Image authors should keep in mind the possibility that a decoder will ignore transparency control. Hence, the colors assigned to transparent pixels should be reasonable background colors whenever feasible.

For applications that do not require a full alpha channel, or cannot afford the price in compression efficiency, the tRNS transparency chunk is also available. 
If the image has a known background color, this color should be written in the bKGD chunk. Even decoders that ignore transparency may use the bKGD color to fill unused screen area.

If the original image has premultiplied (also called "associated") alpha data, convert it to PNG's non-premultiplied format by dividing each sample value by the corresponding alpha value, then multiplying by the maximum value for the image bit depth, and rounding to the nearest integer. In valid premultiplied data, the sample values never exceed their corresponding alpha values, so the result of the division should always be in the range 0 to 1 . If the alpha value is zero, output black (zeroes).

\subsection{Suggested palettes}

A PLTE chunk can appear in truecolor PNG files. In such files, the chunk is not an essential part of the image data, but simply represents a suggested palette that viewers may use to present the image on indexed-color display hardware. A suggested palette is of no interest to viewers running on truecolor hardware.

If an encoder chooses to provide a suggested palette, it is recommended that a hIST chunk also be written to indicate the relative importance of the palette entries. The histogram values are most easily computed as "nearest neighbor" counts, that is, the approximate usage of each palette entry if no dithering is applied. (These counts will often be available for free as a consequence of developing the suggested palette.)

For images of color type 2 (truecolor without alpha channel), it is recommended that the palette and histogram be computed with reference to the RGB data only, ignoring any transparent-color specification. If the file uses transparency (has a tRNS chunk), viewers can easily adapt the resulting palette for use with their intended background color. They need only replace the palette entry closest to the tRNS color with their background color (which may or may not match the file's bKGD color, if any).

For images of color type 6 (truecolor with alpha channel), it is recommended that a bKGD chunk appear and that the palette and histogram be computed with reference to the image as it would appear after compositing against the specified background color. This definition is necessary to ensure that useful palette entries are generated for pixels having fractional alpha values. The resulting palette will probably only be useful to viewers that present the image against the same background color. It is recommended that PNG editors delete or recompute the palette if they alter or remove the bKGD chunk in an image of color type 6 . 
If PLTE appears without bKGD in an image of color type 6, the circumstances under which the palette was computed are unspecified.

\subsection{Filter selection}

For images of color type 3 (indexed color), filter type 0 (None) is usually the most effective. Note that color images with 256 or fewer colors should almost always be stored in indexed color format; truecolor format is likely to be much larger.

Filter type 0 is also recommended for images of bit depths less than 8. For low-bit-depth grayscale images, it may be a net win to expand the image to 8-bit representation and apply filtering, but this is rare.

For truecolor and grayscale images, any of the five filters may prove the most effective. If an encoder uses a fixed filter, the Paeth filter is most likely to be the best.

For best compression of truecolor and grayscale images, we recommend an adaptive filtering approach in which a filter is chosen for each scanline. The following simple heuristic has performed well in early tests: compute the output scanline using all five filters, and select the filter that gives the smallest sum of absolute values of outputs. (Consider the output bytes as signed differences for this test.) This method usually outperforms any single fixed filter choice. However, it is likely that much better heuristics will be found as more experience is gained with PNG.

Filtering according to these recommendations is effective on interlaced as well as noninterlaced images.

\subsection{Text chunk processing}

A nonempty keyword must be provided for each text chunk. The generic keyword "Comment" can be used if no better description of the text is available. If a user-supplied keyword is used, be sure to check that it meets the restrictions on keywords.

PNG text strings are expected to use the Latin-1 character set. Encoders should avoid storing characters that are not defined in Latin-1, and should provide character code remapping if the local system's character set is not Latin-1.

Encoders should discourage the creation of single lines of text longer than 79 characters, in order to facilitate easy reading. 
It is recommended that text items less than $1 \mathrm{~K}$ (1024 bytes) in size should be output using uncompressed tExt chunks. In particular, it is recommended that the basic title and author keywords should always be output using uncompressed tExt chunks. Lengthy disclaimers, on the other hand, are ideal candidates for zTXt.

Placing large tExt and zTXt chunks after the image data (after IDAT) can speed up image display in some situations, since the decoder won't have to read over the text to get to the image data. But it is recommended that small text chunks, such as the image title, appear before IDAT.

\subsection{Use of private chunks}

Applications can use PNG private chunks to carry information that need not be understood by other applications. Such chunks must be given names with lowercase second letters, to ensure that they can never conflict with any future public chunk definition. Note, however, that there is no guarantee that some other application will not use the same private chunk name. If you use a private chunk type, it is prudent to store additional identifying information at the beginning of the chunk data.

Use an ancillary chunk type (lowercase first letter), not a critical chunk type, for all private chunks that store information that is not absolutely essential to view the image. Creation of private critical chunks is discouraged because they render PNG files unportable. Such chunks should not be used in publicly available software or files. If private critical chunks are essential for your application, it is recommended that one appear near the start of the file, so that a standard decoder need not read very far before discovering that it cannot handle the file.

If you want others outside your organization to understand a chunk type that you invent, contact the maintainers of the PNG specification to submit a proposed chunk name and definition for addition to the list of special-purpose public chunks (see Additional chunk types, Section 4.4). Note that a proposed public chunk name (with uppercase second letter) must not be used in publicly available software or files until registration has been approved.

If an ancillary chunk contains textual information that might be of interest to a human user, you should not create a special chunk type for it. Instead use a tEXt chunk and define a suitable keyword. That way, the information will be available to users not using your software. 
Keywords in tExt chunks should be reasonably self-explanatory, since the idea is to let other users figure out what the chunk contains. If of general usefulness, new keywords can be registered with the maintainers of the PNG specification. But it is permissible to use keywords without registering them first.

9.9. Private type and method codes

This specification defines the meaning of only some of the possible values of some fields. For example, only compression method 0 and filter types 0 through 4 are defined. Numbers greater than 127 must be used when inventing experimental or private definitions of values for any of these fields. Numbers below 128 are reserved for possible future public extensions of this specification. Note that use of private type codes may render a file unreadable by standard decoders. Such codes are strongly discouraged except for experimental purposes, and should not appear in publicly available software or files.

10. Recommendations for Decoders

This chapter gives some recommendations for decoder behavior. The only absolute requirement on a PNG decoder is that it successfully read any file conforming to the format specified in the preceding chapters. However, best results will usually be achieved by following these recommendations.

\subsection{Error checking}

To ensure early detection of common file-transfer problems, decoders should verify that all eight bytes of the PNG file signature are correct. (See Rationale: PNG file signature, Section 12.11.) A decoder can have additional confidence in the file's integrity if the next eight bytes are an IHDR chunk header with the correct chunk length.

Unknown chunk types must be handled as described in Chunk naming conventions (Section 3.3). An unknown chunk type is not to be treated as an error unless it is a critical chunk.

It is strongly recommended that decoders should verify the CRC on each chunk.

In some situations it is desirable to check chunk headers (length and type code) before reading the chunk data and CRC. The chunk type can be checked for plausibility by seeing whether all four bytes are ASCII letters (codes 65-90 and 97-122); note that this need only be done for unrecognized type codes. If the total file 
size is known (from file system information, HTTP protocol, etc), the chunk length can be checked for plausibility as well.

If CRCs are not checked, dropped/added data bytes or an erroneous chunk length can cause the decoder to get out of step and misinterpret subsequent data as a chunk header. Verifying that the chunk type contains letters is an inexpensive way of providing early error detection in this situation.

For known-length chunks such as IHDR, decoders should treat an unexpected chunk length as an error. Future extensions to this specification will not add new fields to existing chunks; instead, new chunk types will be added to carry new information.

Unexpected values in fields of known chunks (for example, an unexpected compression method in the IHDR chunk) must be checked for and treated as errors. However, it is recommended that unexpected field values be treated as fatal errors only in critical chunks. An unexpected value in an ancillary chunk can be handled by ignoring the whole chunk as though it were an unknown chunk type. (This recommendation assumes that the chunk's CRC has been verified. In decoders that do not check CRCs, it is safer to treat any unexpected value as indicating a corrupted file.)

\subsection{Pixel dimensions}

Non-square pixels can be represented (see the pHYs chunk), but viewers are not required to account for them; a viewer can present any PNG file as though its pixels are square.

Conversely, viewers running on display hardware with non-square pixels are strongly encouraged to rescale images for proper display.

\subsection{Truecolor image handling}

To achieve PNG's goal of universal interchangeability, decoders are required to accept all types of PNG image: indexed-color, truecolor, and grayscale. Viewers running on indexed-color display hardware need to be able to reduce truecolor images to indexed format for viewing. This process is usually called "color quantization". 
A simple, fast way of doing this is to reduce the image to a fixed palette. Palettes with uniform color spacing ("color cubes") are usually used to minimize the per-pixel computation. For photograph-like images, dithering is recommended to avoid ugly contours in what should be smooth gradients; however, dithering introduces graininess that can be objectionable.

The quality of rendering can be improved substantially by using a palette chosen specifically for the image, since a color cube usually has numerous entries that are unused in any particular image. This approach requires more work, first in choosing the palette, and second in mapping individual pixels to the closest available color. PNG allows the encoder to supply a suggested palette in a PLTE chunk, but not all encoders will do so, and the suggested palette may be unsuitable in any case (it may have too many or too few colors). High-quality viewers will therefore need to have a palette selection routine at hand. A large lookup table is usually the most feasible way of mapping individual pixels to palette entries with adequate speed.

Numerous implementations of color quantization are available. The PNG reference implementation, libpng, includes code for the purpose.

10.4. Sample depth rescaling

Decoders may wish to scale PNG data to a lesser sample depth (data precision) for display. For example, 16-bit data will need to be reduced to 8-bit depth for use on most present-day display hardware. Reduction of 8-bit data to 5-bit depth is also common.

The most accurate scaling is achieved by the linear equation

output $=$ ROUND (input * MAXOUTSAMPLE / MAXINSAMPLE)

where

MAXINSAMPLE $=\left(2^{\wedge}\right.$ sampledepth $)-1$

MAXOUTSAMPLE $=\left(2^{\wedge}\right.$ desired_sampledept $\left.h\right)-1$

A slightly less accurate conversion is achieved by simply shifting right by sampledepth-desired_sampledepth places. For example, to reduce 16-bit samples to 8-bit, one need only discard the loworder byte. In many situations the shift method is sufficiently accurate for display purposes, and it is certainly much faster. (But if gamma correction is being done, sample rescaling can be merged into the gamma correction lookup table, as is illustrated in Decoder gamma handling, Section 10.5.) 
When an sBIT chunk is present, the original pre-PNG data can be recovered by shifting right to the sample depth specified by SBIT. Note that linear scaling will not necessarily reproduce the original data, because the encoder is not required to have used linear scaling to scale the data up. However, the encoder is required to have used a method that preserves the high-order bits, so shifting always works. This is the only case in which shifting might be said to be more accurate than linear scaling.

When comparing pixel values to tRNS chunk values to detect transparent pixels, it is necessary to do the comparison exactly. Therefore, transparent pixel detection must be done before reducing sample precision.

10.5. Decoder gamma handling

See Gamma Tutorial (Chapter 13) if you aren't already familiar with gamma issues.

To produce correct tone reproduction, a good image display program should take into account the gammas of the image file and the display device, as well as the viewing_gamma appropriate to the lighting conditions near the display. This can be done by calculating

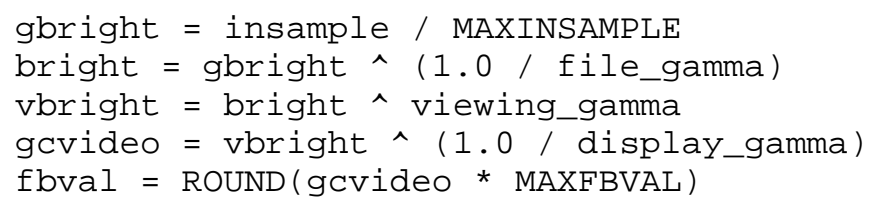

where MAXINSAMPLE is the maximum sample value in the file (255 for 8-bit, 65535 for 16-bit, etc), MAXFBVAL is the maximum value of a frame buffer sample (255 for 8-bit, 31 for 5-bit, etc), insample is the value of the sample in the PNG file, and fbval is the value to write into the frame buffer. The first line converts from integer samples into a normalized 0 to 1 floating point value, the second undoes the gamma encoding of the image file to produce a linear intensity value, the third adjusts for the viewing conditions, the fourth corrects for the display system's gamma value, and the fifth converts to an integer frame buffer sample. In practice, the second through fourth lines can be merged into gcvideo = gbright^(viewing_gamma / (file_gamma*display_gamma))

so as to perform only one power calculation. For color images, the entire calculation is performed separately for R, G, and B values. 
It is not necessary to perform transcendental math for every pixel. Instead, compute a lookup table that gives the correct output value for every possible sample value. This requires only 256 calculations per image (for 8-bit accuracy), not one or three calculations per pixel. For an indexed-color image, a one-time correction of the palette is sufficient, unless the image uses transparency and is being displayed against a nonuniform background.

In some cases even the cost of computing a gamma lookup table may be a concern. In these cases, viewers are encouraged to have precomputed gamma correction tables for file_gamma values of 1.0 and 0.5 with some reasonable choice of viewing_gamma and display_gamma, and to use the table closest to the gamma indicated in the file. This will produce acceptable results for the majority of real files.

When the incoming image has unknown gamma (no gAMA chunk), choose a likely default file_gamma value, but allow the user to select a new one if the result proves too dark or too light.

In practice, it is often difficult to determine what value of display_gamma should be used. In systems with no built-in gamma correction, the display_gamma is determined entirely by the CRT. Assuming a CRT_gamma of 2.5 is recommended, unless you have detailed calibration measurements of this particular CRT available.

However, many modern frame buffers have lookup tables that are used to perform gamma correction, and on these systems the display_gamma value should be the gamma of the lookup table and CRT combined. You may not be able to find out what the lookup table contains from within an image viewer application, so you may have to ask the user what the system's gamma value is. Unfortunately, different manufacturers use different ways of specifying what should go into the lookup table, so interpretation of the system gamma value is system-dependent. Gamma Tutorial (Chapter 13) gives some examples.

The response of real displays is actually more complex than can be described by a single number (display_gamma). If actual measurements of the monitor's light output as a function of voltage input are available, the fourth and fifth lines of the computation above can be replaced by a lookup in these measurements, to find the actual frame buffer value that most nearly gives the desired brightness. 
The value of viewing_gamma depends on lighting conditions; see Gamma Tutorial (Chapter 13) for more detail. Ideally, a viewer would allow the user to specify viewing_gamma, either directly numerically, or via selecting from "bright surround", "dim surround", and "dark surround" conditions. Viewers that don't want to do this should just assume a value for viewing_gamma of 1.0, since most computer displays live in brightly-lit rooms.

When viewing images that are digitized from video, or that are destined to become video frames, the user might want to set the viewing_gamma to about 1.25 regardless of the actual level of room lighting. This value of viewing_gamma is "built into" NTSC video practice, and displaying an image with that viewing_gamma allows the user to see what a TV set would show under the current room lighting conditions. (This is not the same thing as trying to obtain the most accurate rendition of the content of the scene, which would require adjusting viewing_gamma to correspond to the room lighting level.) This is another reason viewers might want to allow users to adjust viewing_gamma directly.

10.6. Decoder color handling

See Color Tutorial (Chapter 14) if you aren't already familiar with color issues.

In many cases, decoders will treat image data in PNG files as device-dependent RGB data and display it without modification (except for appropriate gamma correction). This provides the fastest display of PNG images. But unless the viewer uses exactly the same display hardware as the original image author used, the colors will not be exactly the same as the original author saw, particularly for darker or near-neutral colors. The cHRM chunk provides information that allows closer color matching than that provided by gamma correction alone.

Decoders can use the CHRM data to transform the image data from RGB to XYZ and thence into a perceptually linear color space such as CIE LAB. They can then partition the colors to generate an optimal palette, because the geometric distance between two colors in CIE LAB is strongly related to how different those colors appear (unlike, for example, RGB or XYZ spaces). The resulting palette of colors, once transformed back into RGB color space, could be used for display or written into a PLTE chunk.

Decoders that are part of image processing applications might also transform image data into CIE LAB space for analysis. 
In applications where color fidelity is critical, such as product design, scientific visualization, medicine, architecture, or advertising, decoders can transform the image data from source_RGB to the display_RGB space of the monitor used to view the image. This involves calculating the matrix to go from source_RGB to XYZ and the matrix to go from XYZ to display_RGB, then combining them to produce the overall transformation. The decoder is responsible for implementing gamut mapping.

Decoders running on platforms that have a Color Management system (CMS) can pass the image data, gAMA and CHRM values to the CMS for display or further processing.

Decoders that provide color printing facilities can use the facilities in Level 2 postscript to specify image data in calibrated RGB space or in a device-independent color space such as XYZ. This will provide better color fidelity than a simple RGB to CMYK conversion. The PostScript Language Reference manual gives examples of this process [POSTSCRIPT]. Such decoders are responsible for implementing gamut mapping between source_RGB (specified in the cHRM chunk) and the target printer. The PostScript interpreter is then responsible for producing the required colors.

Decoders can use the cHRM data to calculate an accurate grayscale representation of a color image. Conversion from RGB to gray is simply a case of calculating the Y (luminance) component of XYZ, which is a weighted sum of the $R$ G and $B$ values. The weights depend on the monitor type, i.e., the values in the cHRM chunk. Decoders may wish to do this for PNG files with no cHRM chunk. In that case, a reasonable default would be the CCIR 709 primaries [ITU-BT709]. Do not use the original NTSC primaries, unless you really do have an image color-balanced for such a monitor. Few monitors ever used the NTSC primaries, so such images are probably nonexistent these days.

\subsection{Background color}

The background color given by bKGD will typically be used to fill unused screen space around the image, as well as any transparent pixels within the image. (Thus, bKGD is valid and useful even when the image does not use transparency.) If no bKGD chunk is present, the viewer will need to make its own decision about a suitable background color. 
Viewers that have a specific background against which to present the image (such as Web browsers) should ignore the bKGD chunk, in effect overriding bKGD with their preferred background color or background image.

The background color given by bKGD is not to be considered transparent, even if it happens to match the color given by tRNS (or, in the case of an indexed-color image, refers to a palette index that is marked as transparent by tRNS). Otherwise one would have to imagine something "behind the background" to composite against. The background color is either used as background or ignored; it is not an intermediate layer between the PNG image and some other background.

Indeed, it will be common that bKGD and tRNS specify the same color, since then a decoder that does not implement transparency processing will give the intended display, at least when no partially-transparent pixels are present.

\subsection{Alpha channel processing}

In the most general case, the alpha channel can be used to composite a foreground image against a background image; the PNG file defines the foreground image and the transparency mask, but not the background image. Decoders are not required to support this most general case. It is expected that most will be able to support compositing against a single background color, however.

The equation for computing a composited sample value is

$$
\text { output }=\text { alpha } * \text { foreground }+(1-a l p h a) * \text { background }
$$

where alpha and the input and output sample values are expressed as fractions in the range 0 to 1 . This computation should be performed with linear (non-gamma-encoded) sample values. For color images, the computation is done separately for $R, G$, and $B$ samples.

The following code illustrates the general case of compositing a foreground image over a background image. It assumes that you have the original pixel data available for the background image, and that output is to a frame buffer for display. Other variants are possible; see the comments below the code. The code allows the sample depths and gamma values of foreground image, background image, and frame buffer/CRT all to be different. Don't assume they are the same without checking. 
This code is standard $\mathrm{C}$, with line numbers added for reference in the comments below.

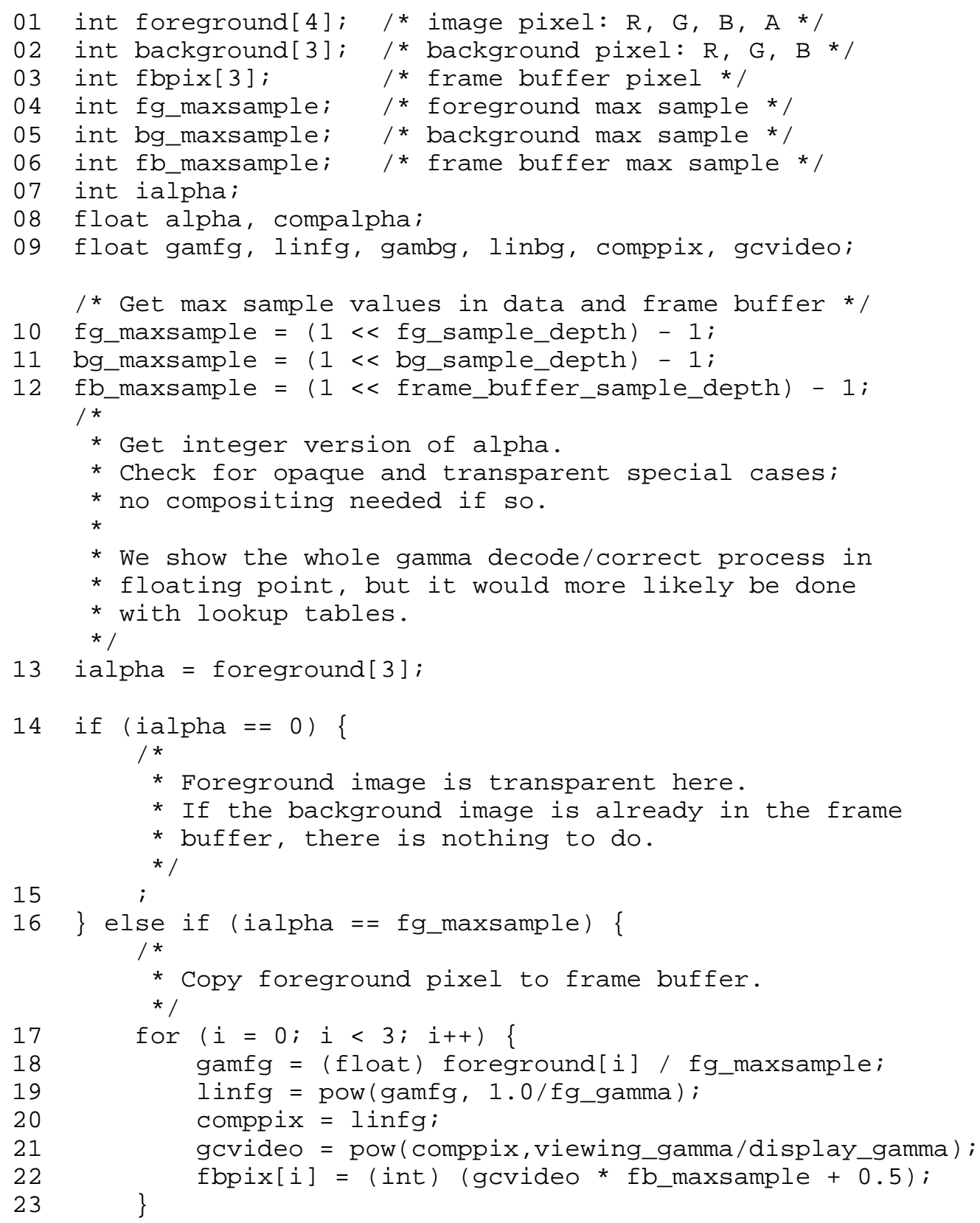


* If the sample depths of the output file, foreground file, and background file are all the same, and the three gamma values also match, then the no-compositing code in lines 14-23 reduces to nothing more than copying pixel values from the input file to the output file if alpha is one, or copying pixel values from background to output file if alpha is zero. Since alpha is typically either zero or one for the vast majority of pixels in an image, this is a great savings. No gamma computations are needed for most pixels.

* When the sample depths and gamma values all match, it may appear attractive to skip the gamma decoding and encoding (lines 28-31, 33-34) and just perform line 32 using gammaencoded sample values. Although this doesn't hurt image quality too badly, the time savings are small if alpha values of zero and one are special-cased as recommended here.

* If the original pixel values of the background image are no longer available, only processed frame buffer pixels left by display of the background image, then lines 30 and 31 need to extract intensity from the frame buffer pixel values using code like

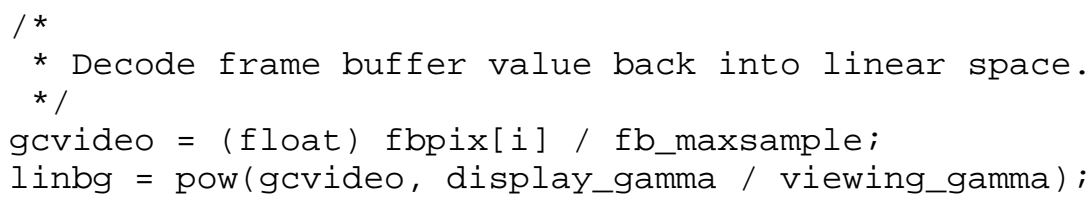

* Note that lines 18-22 are performing exactly the same gamma computation that is done when no alpha channel is present. So, if you handle the no-alpha case with a lookup table, you can use the same lookup table here. Lines 28-31 and 33-34 can also be done with (different) lookup tables.

* Of course, everything here can be done in integer arithmetic. Just be careful to maintain sufficient precision all the way through.

Note: in floating point, no overflow or underflow checks are needed, because the input sample values are guaranteed to be between 0 and 1 , and compositing always yields a result that is in between the input values (inclusive). With integer arithmetic, some roundoff-error analysis might be needed to guarantee no overflow or underflow. 
When displaying a PNG image with full alpha channel, it is important to be able to composite the image against some background, even if it's only black. Ignoring the alpha channel will cause PNG images that have been converted from an associated-alpha representation to look wrong. (Of course, if the alpha channel is a separate transparency mask, then ignoring alpha is a useful option: it allows the hidden parts of the image to be recovered.)

Even if the decoder author does not wish to implement true compositing logic, it is simple to deal with images that contain only zero and one alpha values. (This is implicitly true for grayscale and truecolor PNG files that use a tRNS chunk; for indexed-color PNG files, it is easy to check whether tRNS contains any values other than 0 and 255.) In this simple case, transparent pixels are replaced by the background color, while others are unchanged. If a decoder contains only this much transparency capability, it should deal with a full alpha channel by treating all nonzero alpha values as fully opaque; that is, do not replace partially transparent pixels by the background. This approach will not yield very good results for images converted from associated-alpha formats, but it's better than doing nothing.

10.9. Progressive display

When receiving images over slow transmission links, decoders can improve perceived performance by displaying interlaced images progressively. This means that as each pass is received, an approximation to the complete image is displayed based on the data received so far. One simple yet pleasing effect can be obtained by expanding each received pixel to fill a rectangle covering the yet-to-be-transmitted pixel positions below and to the right of the received pixel. This process can be described by the following pseudocode:

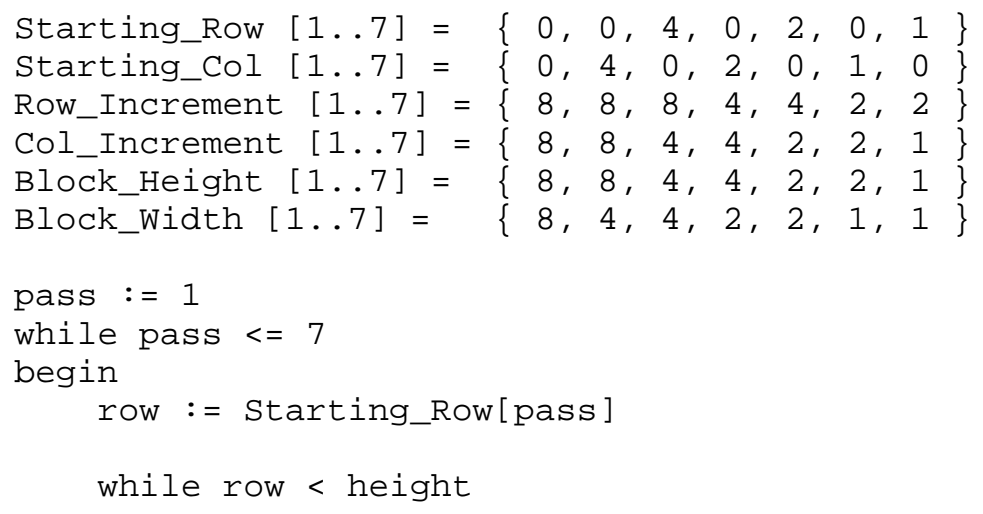




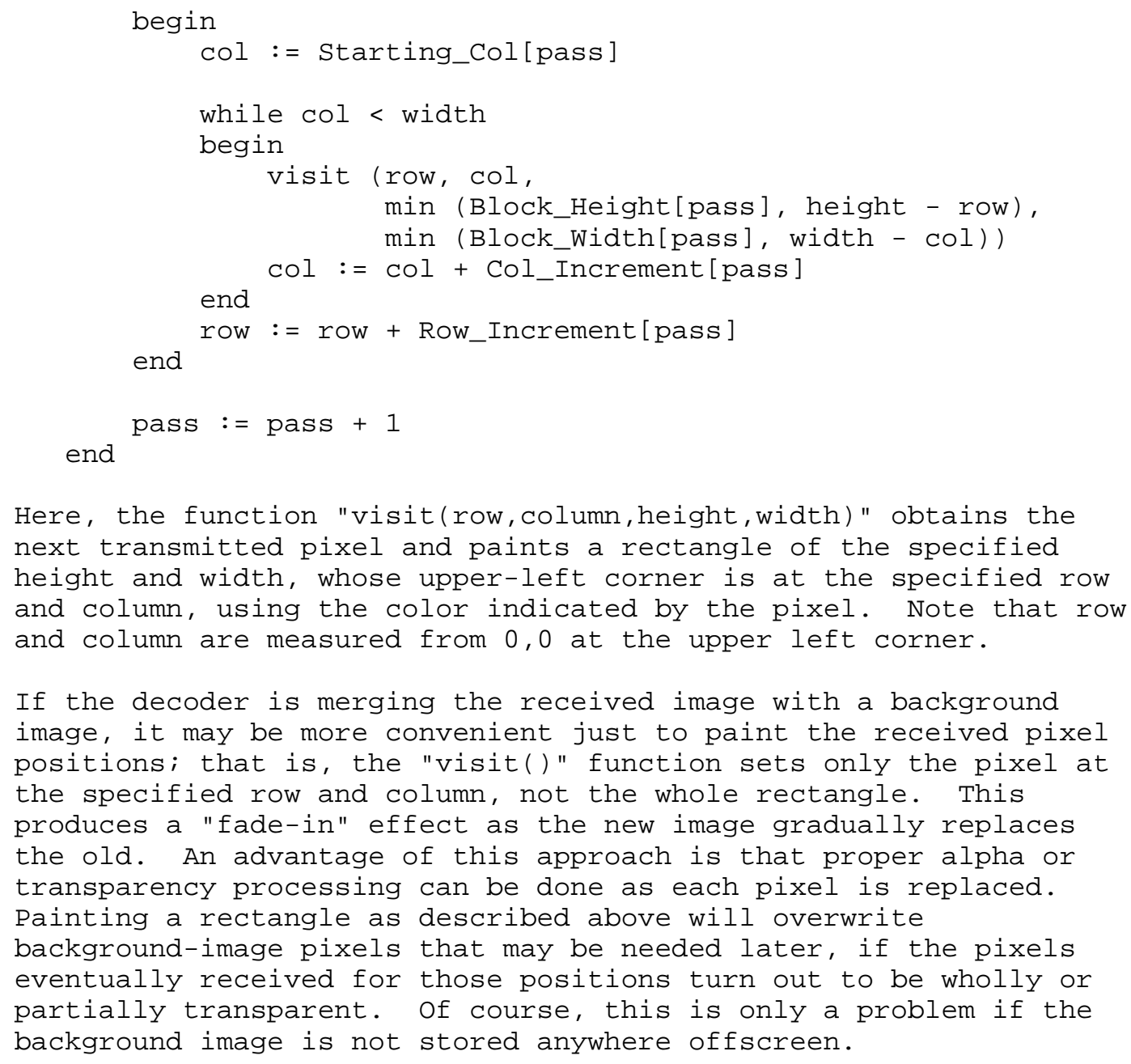

10.10. Suggested-palette and histogram usage

In truecolor PNG files, the encoder may have provided a suggested PLTE chunk for use by viewers running on indexed-color hardware.

If the image has a tRNS chunk, the viewer will need to adapt the suggested palette for use with its desired background color. To do this, replace the palette entry closest to the tRNS color with the desired background color; or just add a palette entry for the background color, if the viewer can handle more colors than there are PLTE entries. 
For images of color type 6 (truecolor with alpha channel), any suggested palette should have been designed for display of the image against a uniform background of the color specified by bKGD. Viewers should probably ignore the palette if they intend to use a different background, or if the bKGD chunk is missing. Viewers can use a suggested palette for display against a different background than it was intended for, but the results may not be very good.

If the viewer presents a transparent truecolor image against a background that is more complex than a single color, it is unlikely that the suggested palette will be optimal for the composite image. In this case it is best to perform a truecolor compositing step on the truecolor PNG image and background image, then color-quantize the resulting image.

The histogram chunk is useful when the viewer cannot provide as many colors as are used in the image's palette. If the viewer is only short a few colors, it is usually adequate to drop the least-used colors from the palette. To reduce the number of colors substantially, it's best to choose entirely new representative colors, rather than trying to use a subset of the existing palette. This amounts to performing a new color quantization step; however, the existing palette and histogram can be used as the input data, thus avoiding a scan of the image data.

If no palette or histogram chunk is provided, a decoder can develop its own, at the cost of an extra pass over the image data. Alternatively, a default palette (probably a color cube) can be used.

See also Recommendations for Encoders: Suggested palettes (Section $9.5)$.

10.11. Text chunk processing

If practical, decoders should have a way to display to the user all tEXt and zTXt chunks found in the file. Even if the decoder does not recognize a particular text keyword, the user might be able to understand it.

PNG text is not supposed to contain any characters outside the ISO 8859-1 "Latin-1" character set (that is, no codes 0-31 or 127159), except for the newline character (decimal 10). But decoders might encounter such characters anyway. Some of these characters can be safely displayed (e.g., TAB, FF, and CR, decimal 9, 12, and 13, respectively), but others, especially the ESC character (decimal 27), could pose a security hazard because unexpected 
actions may be taken by display hardware or software. To prevent such hazards, decoders should not attempt to directly display any non-Latin-1 characters (except for newline and perhaps TAB, FF, CR) encountered in a tEXt or zTXt chunk. Instead, ignore them or display them in a visible notation such as "\nnn". See Security considerations (Section 8.5).

Even though encoders are supposed to represent newlines as LF, it is recommended that decoders not rely on this; it's best to recognize all the common newline combinations (CR, LF, and CR-LF) and display each as a single newline. TAB can be expanded to the proper number of spaces needed to arrive at a column multiple of 8 .

Decoders running on systems with non-Latin-1 character set encoding should provide character code remapping so that Latin-1 characters are displayed correctly. Some systems may not provide all the characters defined in Latin-1. Mapping unavailable characters to a visible notation such as "\nnn" is a good fallback. In particular, character codes 127-255 should be displayed only if they are printable characters on the decoding system. Some systems may interpret such codes as control characters; for security, decoders running on such systems should not display such characters literally.

Decoders should be prepared to display text chunks that contain any number of printing characters between newline characters, even though encoders are encouraged to avoid creating lines in excess of 79 characters.

11. Glossary

$a^{\wedge} b$

Exponentiation; a raised to the power b. C programmers should be careful not to misread this notation as exclusive-or. Note that in gamma-related calculations, zero raised to any power is valid and must give a zero result.

Alpha

A value representing a pixel's degree of transparency. The more transparent a pixel, the less it hides the background against which the image is presented. In PNG, alpha is really the degree of opacity: zero alpha represents a completely transparent pixel, maximum alpha represents a completely opaque pixel. But most people refer to alpha as providing transparency information, not opacity information, and we continue that custom here. 
Ancillary chunk

A chunk that provides additional information. A decoder can still produce a meaningful image, though not necessarily the best possible image, without processing the chunk.

Bit depth

The number of bits per palette index (in indexed-color PNGs) or per sample (in other color types). This is the same value that appears in IHDR.

Byte

Eight bits; also called an octet.

Channel

The set of all samples of the same kind within an image; for example, all the blue samples in a truecolor image. (The term "component" is also used, but not in this specification.) A sample is the intersection of a channel and a pixel.

Chromaticity

A pair of values $x, y$ that precisely specify the hue, though not the absolute brightness, of a perceived color.

Chunk

A section of a PNG file. Each chunk has a type indicated by its chunk type name. Most types of chunks also include some data. The format and meaning of the data within the chunk are determined by the type name.

Composite

As a verb, to form an image by merging a foreground image and a background image, using transparency information to determine where the background should be visible. The foreground image is said to be "composited against" the background.

$\mathrm{CRC}$

Cyclic Redundancy Check. A CRC is a type of check value designed to catch most transmission errors. A decoder calculates the CRC for the received data and compares it to the CRC that the encoder calculated, which is appended to the data. A mismatch indicates that the data was corrupted in transit.

Critical chunk

A chunk that must be understood and processed by the decoder in order to produce a meaningful image from a PNG file.

CRT

Cathode Ray Tube: a common type of computer display hardware. 


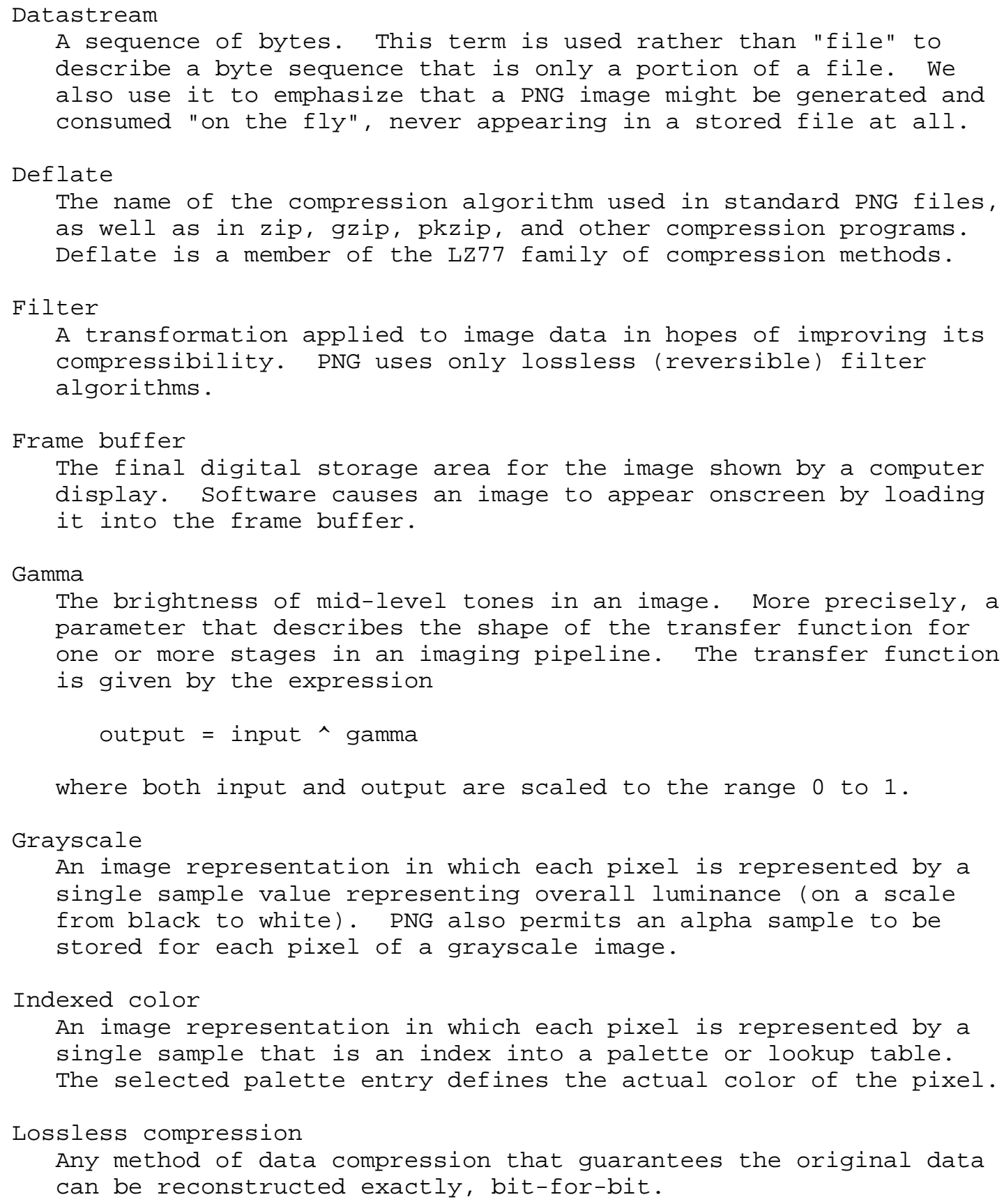


Lossy compression

Any method of data compression that reconstructs the original data approximately, rather than exactly.

LSB

Least Significant Byte of a multi-byte value.

Luminance

Perceived brightness, or grayscale level, of a color. Luminance and chromaticity together fully define a perceived color.

LUT

Look Up Table. In general, a table used to transform data. In

frame buffer hardware, a LUT can be used to map indexed-color pixels into a selected set of truecolor values, or to perform gamma correction. In software, a LUT can be used as a fast way of implementing any one-variable mathematical function.

MSB

Most Significant Byte of a multi-byte value.

Palette

The set of colors available in an indexed-color image. In PNG, a palette is an array of colors defined by red, green, and blue samples. (Alpha values can also be defined for palette entries, via the tRNS chunk.)

Pixel

The information stored for a single grid point in the image. The complete image is a rectangular array of pixels.

PNG editor

A program that modifies a PNG file and preserves ancillary information, including chunks that it does not recognize. Such a program must obey the rules given in Chunk Ordering Rules (Chapter 7).

Sample

A single number in the image data; for example, the red value of a pixel. A pixel is composed of one or more samples. When discussing physical data layout (in particular, in Image layout, Section 2.3), we use "sample" to mean a number stored in the image array. It would be more precise but much less readable to say "sample or palette index" in that context. Elsewhere in the specification, "sample" means a color value or alpha value. In the indexed-color case, these are palette entries not palette indexes. 
Sample depth

The precision, in bits, of color values and alpha values. In indexed-color PNGs the sample depth is always 8 by definition of the PLTE chunk. In other color types it is the same as the bit depth.

Scanline

One horizontal row of pixels within an image.

Truecolor

An image representation in which pixel colors are defined by storing three samples for each pixel, representing red, green, and blue intensities respectively. PNG also permits an alpha sample to be stored for each pixel of a truecolor image.

White point The chromaticity of a computer display's nominal white value.

zlib

A particular format for data that has been compressed using deflate-style compression. Also the name of a library implementing this method. PNG implementations need not use the zlib library, but they must conform to its format for compressed data.

\section{Appendix: Rationale}

(This appendix is not part of the formal PNG specification.)

This appendix gives the reasoning behind some of the design decisions in PNG. Many of these decisions were the subject of considerable debate. The authors freely admit that another group might have made different decisions; however, we believe that our choices are defensible and consistent.

12.1. Why a new file format?

Does the world really need yet another graphics format? We believe so. GIF is no longer freely usable, but no other commonly used format can directly replace it, as is discussed in more detail below. We might have used an adaptation of an existing format, for example GIF with an unpatented compression scheme. But this would require new code anyway; it would not be all that much easier to implement than a whole new file format. (PNG is designed to be simple to implement, with the exception of the compression engine, which would be needed in any case.) We feel that this is an excellent opportunity to design a new format that fixes some of the known limitations of GIF. 
12.2. Why these features?

The features chosen for PNG are intended to address the needs of applications that previously used the special strengths of GIF. In particular, GIF is well adapted for online communications because of its streamability and progressive display capability. PNG shares those attributes.

We have also addressed some of the widely known shortcomings of GIF. In particular, PNG supports truecolor images. We know of no widely used image format that losslessly compresses truecolor images as effectively as PNG does. We hope that PNG will make use of truecolor images more practical and widespread.

Some form of transparency control is desirable for applications in which images are displayed against a background or together with other images. GIF provided a simple transparent-color specification for this purpose. PNG supports a full alpha channel as well as transparent-color specifications. This allows both highly flexible transparency and compression efficiency.

Robustness against transmission errors has been an important consideration. For example, images transferred across Internet are often mistakenly processed as text, leading to file corruption. PNG is designed so that such errors can be detected quickly and reliably.

PNG has been expressly designed not to be completely dependent on a single compression technique. Although deflate/inflate compression is mentioned in this document, PNG would still exist without it.

\subsection{Why not these features?}

Some features have been deliberately omitted from PNG. These choices were made to simplify implementation of PNG, promote portability and interchangeability, and make the format as simple and foolproof as possible for users. In particular: 
* There is no uncompressed variant of PNG. It is possible to store uncompressed data by using only uncompressed deflate blocks (a feature normally used to guarantee that deflate does not make incompressible data much larger). However, PNG software must support full deflate/inflate; any software that does not is not compliant with the PNG standard. The two most important features of PNG---portability and compression---are absolute requirements for online applications, and users demand them. Failure to support full deflate/inflate compromises both of these objectives.

* There is no lossy compression in PNG. Existing formats such as JFIF already handle lossy compression well. Furthermore, available lossy compression methods (e.g., JPEG) are far from foolproof --- a poor choice of quality level can ruin an image. To avoid user confusion and unintentional loss of information, we feel it is best to keep lossy and lossless formats strictly separate. Also, lossy compression is complex to implement. Adding JPEG support to a PNG decoder might increase its size by an order of magnitude. This would certainly cause some decoders to omit support for the feature, which would destroy our goal of interchangeability.

* There is no support for CMYK or other unusual color spaces. Again, this is in the name of promoting portability. CMYK, in particular, is far too device-dependent to be useful as a portable image representation.

* There is no standard chunk for thumbnail views of images. In discussions with software vendors who use thumbnails in their products, it has become clear that most would not use a "standard" thumbnail chunk. For one thing, every vendor has a different idea of what the dimensions and characteristics of a thumbnail ought to be. Also, some vendors keep thumbnails in separate files to accommodate varied image formats; they are not going to stop doing that simply because of a thumbnail chunk in one new format. Proprietary chunks containing vendor-specific thumbnails appear to be more practical than a common thumbnail format.

It is worth noting that private extensions to PNG could easily add these features. We will not, however, include them as part of the basic PNG standard. 
PNG also does not support multiple images in one file. This restriction is a reflection of the reality that many applications do not need and will not support multiple images per file. In any case, single images are a fundamentally different sort of object from sequences of images. Rather than make false promises of interchangeability, we have drawn a clear distinction between single-image and multi-image formats. PNG is a single-image format. (But see Multiple-image extension, Section 8.4.)

12.4. Why not use format $x$ ?

Numerous existing formats were considered before deciding to develop PNG. None could meet the requirements we felt were important for PNG.

GIF is no longer suitable as a universal standard because of legal entanglements. Although just replacing GIF's compression method would avoid that problem, GIF does not support truecolor images, alpha channels, or gamma correction. The spec has more subtle problems too. Only a small subset of the GIF89 spec is actually portable across a variety of implementations, but there is no codification of the most portable part of the spec.

TIFF is far too complex to meet our goals of simplicity and interchangeability. Defining a TIFF subset would meet that objection, but would frustrate users making the reasonable assumption that a file saved as TIFF from their existing software would load into a program supporting our flavor of TIFF. Furthermore, TIFF is not designed for stream processing, has no provision for progressive display, and does not currently provide any good, legally unencumbered, lossless compression method.

IFF has also been suggested, but is not suitable in detail: available image representations are too machine-specific or not adequately compressed. The overall chunk structure of IFF is a useful concept that PNG has liberally borrowed from, but we did not attempt to be bit-for-bit compatible with IFF chunk structure. Again this is due to detailed issues, notably the fact that IFF FORMs are not designed to be serially writable.

Lossless JPEG is not suitable because it does not provide for the storage of indexed-color images. Furthermore, its lossless truecolor compression is often inferior to that of PNG. 
12.5. Byte order

It has been asked why PNG uses network byte order. We have selected one byte ordering and used it consistently. Which order in particular is of little relevance, but network byte order has the advantage that routines to convert to and from it are already available on any platform that supports TCP/IP networking, including all PC platforms. The functions are trivial and will be included in the reference implementation.

12.6. Interlacing

PNG's two-dimensional interlacing scheme is more complex to implement than GIF's line-wise interlacing. It also costs a little more in file size. However, it yields an initial image eight times faster than GIF (the first pass transmits only 1/64th of the pixels, compared to 1/8th for GIF). Although this initial image is coarse, it is useful in many situations. For example, if the image is a World Wide Web imagemap that the user has seen before, PNG's first pass is often enough to determine where to click. The PNG scheme also looks better than GIF's, because horizontal and vertical resolution never differ by more than a factor of two; this avoids the odd "stretched" look seen when interlaced GIFs are filled in by replicating scanlines. Preliminary results show that small text in an interlaced PNG image is typically readable about twice as fast as in an equivalent GIF, i.e., after PNG's fifth pass or $25 \%$ of the image data, instead of after GIF's third pass or $50 \%$. This is again due to PNG'S $^{\prime}$ more balanced increase in resolution.

\subsection{Why gamma?}

It might seem natural to standardize on storing sample values that are linearly proportional to light intensity (that is, have gamma of 1.0). But in fact, it is common for images to have a gamma of less than 1 . There are three good reasons for this:

* For reasons detailed in Gamma Tutorial (Chapter 13), all video cameras apply a "gamma correction" function to the intensity information. This causes the video signal to have a gamma of about 0.5 relative to the light intensity in the original scene. Thus, images obtained by frame-grabbing video already have a gamma of about 0.5 .

* The human eye has a nonlinear response to intensity, so linear encoding of samples either wastes sample codes in bright areas of the image, or provides too few sample codes to avoid banding artifacts in dark areas of the image, or both. At least 12 bits per sample are needed to avoid 
visible artifacts in linear encoding with a 100:1 image intensity range. An image gamma in the range 0.3 to 0.5 allocates sample values in a way that roughly corresponds to the eye's response, so that 8 bits/sample are enough to avoid artifacts caused by insufficient sample precision in almost all images. This makes "gamma encoding" a much better way of storing digital images than the simpler linear encoding.

* Many images are created on PCs or workstations with no gamma correction hardware and no software willing to provide gamma correction either. In these cases, the images have had their lighting and color chosen to look best on this platform --- they can be thought of as having "manual" gamma correction built in. To see what the image author intended, it is necessary to treat such images as having a file_gamma value in the range 0.4-0.6, depending on the room lighting level that the author was working in.

In practice, image gamma values around 1.0 and around 0.5 are both widely found. Older image standards such as GIF often do not account for this fact. The JFIF standard specifies that images in that format should use linear samples, but many JFIF images found on the Internet actually have a gamma somewhere near 0.4 or 0.5 . The variety of images found and the variety of systems that people display them on have led to widespread problems with images appearing "too dark" or "too light".

PNG expects viewers to compensate for image gamma at the time that the image is displayed. Another possible approach is to expect encoders to convert all images to a uniform gamma at encoding time. While that method would speed viewers slightly, it has fundamental flaws:

* Gamma correction is inherently lossy due to quantization and roundoff error. Requiring conversion at encoding time thus causes irreversible loss. Since PNG is intended to be a lossless storage format, this is undesirable; we should store unmodified source data.

* The encoder might not know the source gamma value. If the decoder does gamma correction at viewing time, it can adjust the gamma (change the displayed brightness) in response to feedback from a human user. The encoder has no such recourse.

* Whatever "standard" gamma we settled on would be wrong for some displays. Hence viewers would still need gamma correction capability. 
Since there will always be images with no gamma or an incorrect recorded gamma, good viewers will need to incorporate gamma adjustment code anyway. Gamma correction at viewing time is thus the right way to go.

See Gamma Tutorial (Chapter 13) for more information.

12.8. Non-premultiplied alpha

PNG uses "unassociated" or "non-premultiplied" alpha so that images with separate transparency masks can be stored losslessly. Another common technique, "premultiplied alpha", stores pixel values premultiplied by the alpha fraction; in effect, the image is already composited against a black background. Any image data hidden by the transparency mask is irretrievably lost by that method, since multiplying by a zero alpha value always produces zero.

Some image rendering techniques generate images with premultiplied alpha (the alpha value actually represents how much of the pixel is covered by the image). This representation can be converted to PNG by dividing the sample values by alpha, except where alpha is zero. The result will look good if displayed by a viewer that handles alpha properly, but will not look very good if the viewer ignores the alpha channel.

Although each form of alpha storage has its advantages, we did not want to require all PNG viewers to handle both forms. We standardized on non-premultiplied alpha as being the lossless and more general case.

\subsection{Filtering}

PNG includes filtering capability because filtering can significantly reduce the compressed size of truecolor and grayscale images. Filtering is also sometimes of value on indexed-color images, although this is less common.

The filter algorithms are defined to operate on bytes, rather than pixels; this gains simplicity and speed with very little cost in compression performance. Tests have shown that filtering is usually ineffective for images with fewer than 8 bits per sample, so providing pixelwise filtering for such images would be pointless. For $16 \mathrm{bit/sample} \mathrm{data,} \mathrm{bytewise} \mathrm{filtering} \mathrm{is} \mathrm{nearly}$ as effective as pixelwise filtering, because MSBs are predicted from adjacent MSBs, and LSBs are predicted from adjacent LSBs. 
The encoder is allowed to change filters for each new scanline. This creates no additional complexity for decoders, since a decoder is required to contain defiltering logic for every filter type anyway. The only cost is an extra byte per scanline in the pre-compression datastream. Our tests showed that when the same filter is selected for all scanlines, this extra byte compresses away to almost nothing, so there is little storage cost compared to a fixed filter specified for the whole image. And the potential benefits of adaptive filtering are too great to ignore. Even with the simplistic filter-choice heuristics so far discovered, adaptive filtering usually outperforms fixed filters. In particular, an adaptive filter can change behavior for successive passes of an interlaced image; a fixed filter cannot.

12.10. Text strings

Most graphics file formats include the ability to store some textual information along with the image. But many applications need more than that: they want to be able to store several identifiable pieces of text. For example, a database using PNG files to store medical X-rays would likely want to include patient's name, doctor's name, etc. A simple way to do this in PNG would be to invent new private chunks holding text. The disadvantage of such an approach is that other applications would have no idea what was in those chunks, and would simply ignore them. Instead, we recommend that textual information be stored in standard tExt chunks with suitable keywords. Use of text tells any PNG viewer that the chunk contains text that might be of interest to a human user. Thus, a person looking at the file with another viewer will still be able to see the text, and even understand what it is if the keywords are reasonably selfexplanatory. (To this end, we recommend spelled-out keywords, not abbreviations that will be hard for a person to understand. Saving a few bytes on a keyword is false economy.)

The ISO 8859-1 (Latin-1) character set was chosen as a compromise between functionality and portability. Some platforms cannot display anything more than 7-bit ASCII characters, while others can handle characters beyond the Latin-1 set. We felt that Latin-1 represents a widely useful and reasonably portable character set. Latin-1 is a direct subset of character sets commonly used on popular platforms such as Microsoft Windows and $\mathrm{X}$ Windows. It can also be handled on Macintosh systems with a simple remapping of characters.

There is presently no provision for text employing character sets other than Latin-1. We recognize that the need for other character sets will increase. However, PNG already requires that 
programmers implement a number of new and unfamiliar features, and text representation is not PNG's primary purpose. Since PNG provides for the creation and public registration of new ancillary chunks of general interest, we expect that text chunks for other character sets, such as Unicode, eventually will be registered and increase gradually in popularity.

12.11. PNG file signature

The first eight bytes of a PNG file always contain the following values:

$\begin{array}{lrrrrrrrr}\text { (decimal) } & 137 & 80 & 78 & 71 & 13 & 10 & 26 & 10 \\ \text { (hexadecimal) } & 89 & 50 & 4 \mathrm{e} & 47 & 0 \mathrm{~d} & 0 \mathrm{a} & 1 \mathrm{a} & 0 \mathrm{a} \\ \text { (ASCII C notation) } & \backslash 211 & \mathrm{P} & \mathrm{N} & \mathrm{G} & \backslash \mathrm{r} & \backslash \mathrm{n} & \backslash 032 & \backslash \mathrm{n}\end{array}$

This signature both identifies the file as a PNG file and provides for immediate detection of common file-transfer problems. The first two bytes distinguish PNG files on systems that expect the first two bytes to identify the file type uniquely. The first byte is chosen as a non-ASCII value to reduce the probability that a text file may be misrecognized as a PNG file; also, it catches bad file transfers that clear bit 7. Bytes two through four name the format. The CR-LF sequence catches bad file transfers that alter newline sequences. The control-z character stops file display under MS-DOS. The final line feed checks for the inverse of the CR-LF translation problem.

A decoder may further verify that the next eight bytes contain an IHDR chunk header with the correct chunk length; this will catch bad transfers that drop or alter null (zero) bytes.

Note that there is no version number in the signature, nor indeed anywhere in the file. This is intentional: the chunk mechanism provides a better, more flexible way to handle format extensions, as explained in Chunk naming conventions (Section 12.13).

12.12. Chunk layout

The chunk design allows decoders to skip unrecognized or uninteresting chunks: it is simply necessary to skip the appropriate number of bytes, as determined from the length field.

Limiting chunk length to (2^31)-1 bytes avoids possible problems for implementations that cannot conveniently handle 4-byte unsigned values. In practice, chunks will usually be much shorter than that anyway. 
A separate CRC is provided for each chunk in order to detect badly-transferred images as quickly as possible. In particular, critical data such as the image dimensions can be validated before being used.

The chunk length is excluded from the CRC so that the CRC can be calculated as the data is generated; this avoids a second pass over the data in cases where the chunk length is not known in advance. Excluding the length from the CRC does not create any extra risk of failing to discover file corruption, since if the length is wrong, the CRC check will fail: the CRC will be computed on the wrong set of bytes and then be tested against the wrong value from the file.

\subsection{Chunk naming conventions}

The chunk naming conventions allow safe, flexible extension of the PNG format. This mechanism is much better than a format version number, because it works on a feature-by-feature basis rather than being an overall indicator. Decoders can process newer files if and only if the files use no unknown critical features (as indicated by finding unknown critical chunks). Unknown ancillary chunks can be safely ignored. We decided against having an overall format version number because experience has shown that format version numbers hurt portability as much as they help. Version numbers tend to be set unnecessarily high, leading to older decoders rejecting files that they could have processed (this was a serious problem for several years after the GIF89 spec came out, for example). Furthermore, private extensions can be made either critical or ancillary, and standard decoders should react appropriately; overall version numbers are no help for private extensions.

A hypothetical chunk for vector graphics would be a critical chunk, since if ignored, important parts of the intended image would be missing. A chunk carrying the Mandelbrot set coordinates for a fractal image would be ancillary, since other applications could display the image without understanding what the image represents. In general, a chunk type should be made critical only if it is impossible to display a reasonable representation of the intended image without interpreting that chunk. 
The public/private property bit ensures that any newly defined public chunk type name cannot conflict with proprietary chunks that could be in use somewhere. However, this does not protect users of private chunk names from the possibility that someone else may use the same chunk name for a different purpose. It is a good idea to put additional identifying information at the start of the data for any private chunk type.

When a PNG file is modified, certain ancillary chunks may need to be changed to reflect changes in other chunks. For example, a histogram chunk needs to be changed if the image data changes. If the file editor does not recognize histogram chunks, copying them blindly to a new output file is incorrect; such chunks should be dropped. The safe/unsafe property bit allows ancillary chunks to be marked appropriately.

Not all possible modification scenarios are covered by the safe/unsafe semantics. In particular, chunks that are dependent on the total file contents are not supported. (An example of such a chunk is an index of IDAT chunk locations within the file: adding a comment chunk would inadvertently break the index.) Definition of such chunks is discouraged. If absolutely necessary for a particular application, such chunks can be made critical chunks, with consequent loss of portability to other applications. In general, ancillary chunks can depend on critical chunks but not on other ancillary chunks. It is expected that mutually dependent information should be put into a single chunk.

In some situations it may be unavoidable to make one ancillary chunk dependent on another. Although the chunk property bits are insufficient to represent this case, a simple solution is available: in the dependent chunk, record the CRC of the chunk depended on. It can then be determined whether that chunk has been changed by some other program.

The same technique can be useful for other purposes. For example, if a program relies on the palette being in a particular order, it can store a private chunk containing the CRC of the PLTE chunk. If this value matches when the file is again read in, then it provides high confidence that the palette has not been tampered with. Note that it is not necessary to mark the private chunk unsafe-to-copy when this technique is used; thus, such a private chunk can survive other editing of the file. 
12.14. Palette histograms

A viewer may not be able to provide as many colors as are listed in the image's palette. (For example, some colors could be reserved by a window system.) To produce the best results in this situation, it is helpful to have information about the frequency with which each palette index actually appears, in order to choose the best palette for dithering or to drop the least-used colors. Since images are often created once and viewed many times, it makes sense to calculate this information in the encoder, although it is not mandatory for the encoder to provide it.

Other image formats have usually addressed this problem by specifying that the palette entries should appear in order of frequency of use. That is an inferior solution, because it doesn't give the viewer nearly as much information: the viewer can't determine how much damage will be done by dropping the last few colors. Nor does a sorted palette give enough information to choose a target palette for dithering, in the case that the viewer needs to reduce the number of colors substantially. A palette histogram provides the information needed to choose such a target palette without making a pass over the image data. 


\section{Appendix: Gamma Tutorial}

(This appendix is not part of the formal PNG specification.)

It would be convenient for graphics programmers if all of the components of an imaging system were linear. The voltage coming from an electronic camera would be directly proportional to the intensity (power) of light in the scene, the light emitted by a CRT would be directly proportional to its input voltage, and so on. However, real-world devices do not behave in this way. All CRT displays, almost all photographic film, and many electronic cameras have nonlinear signal-to-light-intensity or intensity-to-signal characteristics.

Fortunately, all of these nonlinear devices have a transfer function that is approximated fairly well by a single type of mathematical function: a power function. This power function has the general equation

$$
\text { output }=\text { input } \wedge \text { gamma }
$$

where ^ denotes exponentiation, and "gamma" (often printed using the Greek letter gamma, thus the name) is simply the exponent of the power function.

By convention, "input" and "output" are both scaled to the range $0 . .1$, with 0 representing black and 1 representing maximum white (or red, etc). Normalized in this way, the power function is completely described by a single number, the exponent "gamma".

So, given a particular device, we can measure its output as a function of its input, fit a power function to this measured transfer function, extract the exponent, and call it gamma. We often say "this device has a gamma of 2.5" as a shorthand for "this device has a power-law response with an exponent of 2.5". We can also talk about the gamma of a mathematical transform, or of a lookup table in a frame buffer, so long as the input and output of the thing are related by the power-law expression above.

How do gammas combine?

Real imaging systems will have several components, and more than one of these can be nonlinear. If all of the components have transfer characteristics that are power functions, then the transfer function of the entire system is also a power function. The exponent (gamma) of the whole system's transfer function is just the product of all of the individual exponents (gammas) of the separate stages in the system. 
Also, stages that are linear pose no problem, since a power function with an exponent of 1.0 is really a linear function. So a linear transfer function is just a special case of a power function, with a gamma of 1.0 .

Thus, as long as our imaging system contains only stages with linear and power-law transfer functions, we can meaningfully talk about the gamma of the entire system. This is indeed the case with most real imaging systems.

What should overall gamma be?

If the overall gamma of an imaging system is 1.0 , its output is linearly proportional to its input. This means that the ratio between the intensities of any two areas in the reproduced image will be the same as it was in the original scene. It might seem that this should always be the goal of an imaging system: to accurately reproduce the tones of the original scene. Alas, that is not the case.

When the reproduced image is to be viewed in "bright surround" conditions, where other white objects nearby in the room have about the same brightness as white in the image, then an overall gamma of 1.0 does indeed give real-looking reproduction of a natural scene. Photographic prints viewed under room light and computer displays in bright room light are typical "bright surround" viewing conditions.

However, sometimes images are intended to be viewed in "dark surround" conditions, where the room is substantially black except for the image. This is typical of the way movies and slides (transparencies) are viewed by projection. Under these circumstances, an accurate reproduction of the original scene results in an image that human viewers judge as "flat" and lacking in contrast. It turns out that the projected image needs to have a gamma of about 1.5 relative to the original scene for viewers to judge it "natural". Thus, slide film is designed to have a gamma of about 1.5 , not 1.0 .

There is also an intermediate condition called "dim surround", where the rest of the room is still visible to the viewer, but is noticeably darker than the reproduced image itself. This is typical of television viewing, at least in the evening, as well as subdued-light computer work areas. In dim surround conditions, the reproduced image needs to have a gamma of about 1.25 relative to the original scene in order to look natural. 
The requirement for boosted contrast (gamma) in dark surround conditions is due to the way the human visual system works, and applies equally well to computer monitors. Thus, a PNG viewer trying to achieve the maximum realism for the images it displays really needs to know what the room lighting conditions are, and adjust the gamma of the displayed image accordingly.

If asking the user about room lighting conditions is inappropriate or too difficult, just assume that the overall gamma

(viewing_gamma as defined below) should be 1.0 or 1.25. That's all that most systems that implement gamma correction do.

What is a CRT's gamma?

All CRT displays have a power-law transfer characteristic with a gamma of about 2.5. This is due to the physical processes involved in controlling the electron beam in the electron gun, and has nothing to do with the phosphor.

An exception to this rule is fancy "calibrated" CRTs that have internal electronics to alter their transfer function. If you have one of these, you probably should believe what the manufacturer tells you its gamma is. But in all other cases, assuming 2.5 is likely to be pretty accurate.

There are various images around that purport to measure gamma, usually by comparing the intensity of an area containing alternating white and black with a series of areas of continuous gray of different intensity. These are usually not reliable. Test images that use a "checkerboard" pattern of black and white are the worst, because a single white pixel will be reproduced considerably darker than a large area of white. An image that uses alternating black and white horizontal lines (such as the "gamma.png" test image at ftp://ftp.uu.net/graphics/png/images/suite/gamma.png) is much better, but even it may be inaccurate at high "picture" settings on some CRTs.

If you have a good photometer, you can measure the actual light output of a CRT as a function of input voltage and fit a power function to the measurements. However, note that this procedure is very sensitive to the $\mathrm{CRT}^{\prime}$ s black level adjustment, somewhat sensitive to its picture adjustment, and also affected by ambient light. Furthermore, CRTs spread some light from bright areas of an image into nearby darker areas; a single bright spot against a black background may be seen to have a "halo". Your measuring technique will need to minimize the effects of this. 
Because of the difficulty of measuring gamma, using either test images or measuring equipment, you're usually better off just assuming gamma is 2.5 rather than trying to measure it.

What is gamma correction?

A CRT has a gamma of 2.5, and we can't change that. To get an overall gamma of 1.0 (or somewhere near that) for an imaging system, we need to have at least one other component of the "image pipeline" that is nonlinear. If, in fact, there is only one nonlinear stage in addition to the CRT, then it's traditional to say that the CRT has a certain gamma, and that the other nonlinear stage provides "gamma correction" to compensate for the CRT. However, exactly where the "correction" is done depends on circumstance.

In all broadcast video systems, gamma correction is done in the camera. This choice was made in the days when television electronics were all analog, and a good gamma-correction circuit was expensive to build. The original NTSC video standard required cameras to have a transfer function with a gamma of 1/2.2, or about 0.45. Recently, a more complex two-part transfer function has been adopted [SMPTE-170M], but its behavior can be well approximated by a power function with a gamma of 0.5 . When the resulting image is displayed on a CRT with a gamma of 2.5 , the image on screen ends up with a gamma of about 1.25 relative to the original scene, which is appropriate for "dim surround" viewing.

These days, video signals are often digitized and stored in computer frame buffers. This works fine, but remember that gamma correction is "built into" the video signal, and so the digitized video has a gamma of about 0.5 relative to the original scene.

Computer rendering programs often produce linear samples. To display these correctly, intensity on the CRT needs to be directly proportional to the sample values in the frame buffer. This can be done with a special hardware lookup table between the frame buffer and the CRT hardware. The lookup table (often called LUT) is loaded with a mapping that implements a power function with a gamma of 0.4, thus providing "gamma correction" for the CRT gamma.

Thus, gamma correction sometimes happens before the frame buffer, sometimes after. As long as images created in a particular environment are always displayed in that environment, everything is fine. But when people try to exchange images, differences in gamma correction conventions often result in images that seem far too bright and washed out, or far too dark and contrasty. 
Gamma-encoded samples are good

So, is it better to do gamma correction before or after the frame buffer?

In an ideal world, sample values would be stored in floating point, there would be lots of precision, and it wouldn't really matter much. But in reality, we're always trying to store images in as few bits as we can.

If we decide to use samples that are linearly proportional to intensity, and do the gamma correction in the frame buffer LUT, it turns out that we need to use at least 12 bits for each of red, green, and blue to have enough precision in intensity. With any less than that, we will sometimes see "contour bands" or "Mach bands" in the darker areas of the image, where two adjacent sample values are still far enough apart in intensity for the difference to be visible.

However, through an interesting coincidence, the human eye's subjective perception of brightness is related to the physical stimulation of light intensity in a manner that is very much like the power function used for gamma correction. If we apply gamma correction to measured (or calculated) light intensity before quantizing to an integer for storage in a frame buffer, we can get away with using many fewer bits to store the image. In fact, 8 bits per color is almost always sufficient to avoid contouring artifacts. This is because, since gamma correction is so closely related to human perception, we are assigning our 256 available sample codes to intensity values in a manner that approximates how visible those intensity changes are to the eye. Compared to a linear-sample image, we allocate fewer sample values to brighter parts of the tonal range and more sample values to the darker portions of the tonal range.

Thus, for the same apparent image quality, images using gammaencoded sample values need only about two-thirds as many bits of storage as images using linear samples. 


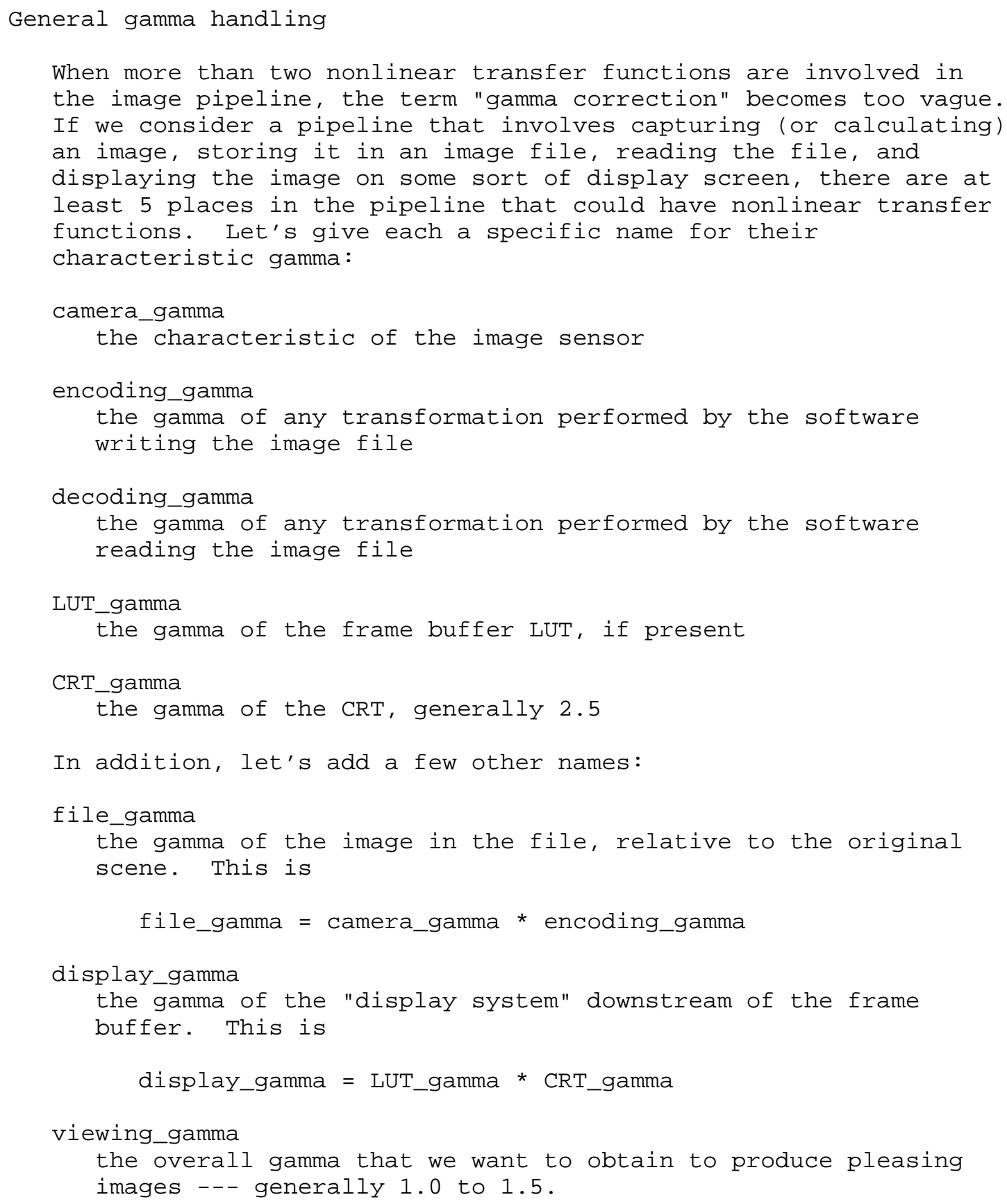


The file_gamma value, as defined above, is what goes in the gAMA chunk in a PNG file. If file_gamma is not 1.0, we know that gamma correction has been done on the sample values in the file, and we could call them "gamma corrected" samples. However, since there can be so many different values of gamma in the image display chain, and some of them are not known at the time the image is written, the samples are not really being "corrected" for a specific display condition. We are really using a power function in the process of encoding an intensity range into a small integer field, and so it is more correct to say "gamma encoded" samples instead of "gamma corrected" samples.

When displaying an image file, the image decoding program is responsible for making the overall gamma of the system equal to the desired viewing_gamma, by selecting the decoding_gamma appropriately. When displaying a PNG file, the gAMA chunk provides the file_gamma value. The display_gamma may be known for this machine, or it might be obtained from the system software, or the user might have to be asked what it is. The correct viewing_gamma depends on lighting conditions, and that will generally have to come from the user.

Ultimately, you should have

file_gamma * decoding_gamma * display_gamma = viewing_gamma

Some specific examples

In digital video systems, camera_gamma is about 0.5 by declaration of the various video standards documents. CRT_gamma is 2.5 as usual, while encoding_gamma, decoding_gamma, and LUT_gamma are all 1.0. As a result, viewing_gamma ends up being about 1.25 .

On frame buffers that have hardware gamma correction tables, and that are calibrated to display linear samples correctly, display_gamma is 1.0 .

Many workstations and X terminals and PC displays lack gamma correction lookup tables. Here, LUT_gamma is always 1.0, so display_gamma is 2.5 . 
On the Macintosh, there is a LUT. By default, it is loaded with a table whose gamma is about 0.72, giving a display_gamma (LUT and CRT combined) of about 1.8. Some Macs have a "Gamma" control panel that allows gamma to be changed to $1.0,1.2,1.4,1.8$, or 2.2. These settings load alternate LUTs that are designed to give a display_gamma that is equal to the label on the selected button. Thus, the "Gamma" control panel setting can be used directly as display_gamma in decoder calculations.

On recent SGI systems, there is a hardware gamma-correction table whose contents are controlled by the (privileged) "gamma" program. The gamma of the table is actually the reciprocal of the number that "gamma" prints, and it does not include the CRT gamma. To obtain the display_gamma, you need to find the SGI system gamma (either by looking in a file, or asking the user) and then calculating

$$
\text { display_gamma }=2.5 / \text { SGI_system_gamma }
$$

You will find SGI systems with the system gamma set to 1.0 and 2.2 (or higher), but the default when machines are shipped is 1.7 .

A note about video gamma

The original NTSC video standards specified a simple power-law camera transfer function with a gamma of $1 / 2.2$ or 0.45 . This is not possible to implement exactly in analog hardware because the function has infinite slope at $\mathrm{x}=0$, so all cameras deviated to some degree from this ideal. More recently, a new camera transfer function that is physically realizable has been accepted as a standard [SMPTE-170M]. It is

$$
\begin{array}{ll}
\text { Vout }=4.5 * \operatorname{Vin} & \text { if } \operatorname{Vin}<0.018 \\
\text { Vout }=1.099 *\left(V_{i n} \wedge 0.45\right)-0.099 & \text { if } \operatorname{Vin}>=0.018
\end{array}
$$

where Vin and Vout are measured on a scale of 0 to 1 . Although the exponent remains 0.45, the multiplication and subtraction change the shape of the transfer function, so it is no longer a pure power function. If you want to perform extremely precise calculations on video signals, you should use the expression above (or its inverse, as required).

However, PNG does not provide a way to specify that an image uses this exact transfer function; the gAMA chunk always assumes a pure power-law function. If we plot the two-part transfer function above along with the family of pure power functions, we find that a power function with a gamma of about 0.5 to 0.52 (not 0.45 ) most closely approximates the transfer function. Thus, when writing a 
PNG file with data obtained from digitizing the output of a modern video camera, the gAMA chunk should contain 0.5 or 0.52 , not 0.45 . The remaining difference between the true transfer function and the power function is insignificant for almost all purposes. (In fact, the alignment errors in most cameras are likely to be larger than the difference between these functions.) The designers of PNG deemed the simplicity and flexibility of a power-law definition of gAMA to be more important than being able to describe the SMPTE-170M transfer curve exactly.

The PAL and SECAM video standards specify a power-law camera transfer function with a gamma of $1 / 2.8$ or $0.36---$ not the $1 / 2.2$ of NTSC. However, this is too low in practice, so real cameras are likely to have their gamma set close to NTSC practice. Just guessing 0.45 or 0.5 is likely to give you viewable results, but if you want precise values you'll probably have to measure the particular camera.

Further reading

If you have access to the World Wide Web, read Charles Poynton's excellent "Gamma FAQ" [GAMMA-FAQ] for more information about gamma.

14. Appendix: Color Tutorial

(This appendix is not part of the formal PNG specification.)

About chromaticity

The cHRM chunk is used, together with the gAMA chunk, to convey precise color information so that a PNG image can be displayed or printed with better color fidelity than is possible without this information. The preceding chapters state how this information is encoded in a PNG image. This tutorial briefly outlines the underlying color theory for those who might not be familiar with it.

Note that displaying an image with incorrect gamma will produce much larger color errors than failing to use the chromaticity data. First be sure the monitor set-up and gamma correction are right, then worry about chromaticity.

The problem

The color of an object depends not only on the precise spectrum of light emitted or reflected from it, but also on the observer --their species, what else they can see at the same time, even what 
they have recently looked at! Furthermore, two very different spectra can produce exactly the same color sensation. Color is not an objective property of real-world objects; it is a subjective, biological sensation. However, by making some simplifying assumptions (such as: we are talking about human vision) it is possible to produce a mathematical model of color and thereby obtain good color accuracy.

Device-dependent color

Display the same RGB data on three different monitors, side by side, and you will get a noticeably different color balance on each display. This is because each monitor emits a slightly different shade and intensity of red, green, and blue light. RGB is an example of a device-dependent color model --- the color you get depends on the device. This also means that a particular color --- represented as say RGB 87, 146, 116 on one monitor --might have to be specified as RGB 98, 123, 104 on another to produce the same color.

Device-independent color

A full physical description of a color would require specifying the exact spectral power distribution of the light source. Fortunately, the human eye and brain are not so sensitive as to require exact reproduction of a spectrum. Mathematical, deviceindependent color models exist that describe fairly well how a particular color will be seen by humans. The most important device-independent color model, to which all others can be related, was developed by the International Lighting Committee (CIE, in French) and is called XYZ.

In XYZ, $X$ is the sum of a weighted power distribution over the whole visible spectrum. So are $Y$ and $Z$, each with different weights. Thus any arbitrary spectral power distribution is condensed down to just three floating point numbers. The weights were derived from color matching experiments done on human subjects in the 1920s. CIE XYZ has been an International standard since 1931, and it has a number of useful properties:

* two colors with the same XYZ values will look the same to humans

* two colors with different XYZ values will not look the same

* the Y value represents all the brightness information (luminance)

* the XYZ color of any object can be objectively measured 
Color models based on XYZ have been used for many years by people who need accurate control of color --- lighting engineers for film and TV, paint and dyestuffs manufacturers, and so on. They are thus proven in industrial use. Accurate, device-independent color started to spread from high-end, specialized areas into the mainstream during the late 1980s and early 1990s, and PNG takes notice of that trend.

Calibrated, device-dependent color

Traditionally, image file formats have used uncalibrated, devicedependent color. If the precise details of the original display device are known, it becomes possible to convert the devicedependent colors of a particular image to device-independent ones. Making simplifying assumptions, such as working with CRTs (which are much easier than printers), all we need to know are the XYZ values of each primary color and the CRT_gamma.

So why does PNG not store images in XYZ instead of RGB? Well, two reasons. First, storing images in XYZ would require more bits of precision, which would make the files bigger. Second, all programs would have to convert the image data before viewing it. Whether calibrated or not, all variants of RGB are close enough that undemanding viewers can get by with simply displaying the data without color correction. By storing calibrated RGB, PNG retains compatibility with existing programs that expect RGB data, yet provides enough information for conversion to XYZ in applications that need precise colors. Thus, we get the best of both worlds.

What are chromaticity and luminance?

Chromaticity is an objective measurement of the color of an object, leaving aside the brightness information. Chromaticity uses two parameters $\mathrm{x}$ and $\mathrm{y}$, which are readily calculated from $\mathrm{XYZ}$ :

$$
\begin{aligned}
& X=X /(X+Y+Z) \\
& Y=Y /(X+Y+Z)
\end{aligned}
$$

XYZ colors having the same chromaticity values will appear to have the same hue but can vary in absolute brightness. Notice that $x, y$ are dimensionless ratios, so they have the same values no matter what units we've used for $X, Y, Z$. 
The $Y$ value of an XYZ color is directly proportional to its absolute brightness and is called the luminance of the color. We can describe a color either by XYZ coordinates or by chromaticity $x, y$ plus luminance $Y$. The XYZ form has the advantage that it is linearly related to (linear, gamma=1.0) RGB color spaces.

How are computer monitor colors described?

The "white point" of a monitor is the chromaticity $x, y$ of the monitor's nominal white, that is, the color produced when $\mathrm{R}=\mathrm{G}=\mathrm{B}=$ maximum.

It's customary to specify monitor colors by giving the chromaticities of the individual phosphors R, G, and B, plus the white point. The white point allows one to infer the relative brightnesses of the three phosphors, which isn't determined by their chromaticities alone.

Note that the absolute brightness of the monitor is not specified. For computer graphics work, we generally don't care very much about absolute brightness levels. Instead of dealing with absolute XYZ values (in which X,Y,Z are expressed in physical units of radiated power, such as candelas per square meter), it is convenient to work in "relative XYZ" units, where the monitor's nominal white is taken to have a luminance (Y) of 1.0. Given this assumption, it's simple to compute XYZ coordinates for the monitor's white, red, green, and blue from their chromaticity values.

Why does cHRM use $x, y$ rather than XYZ? Simply because that is how manufacturers print the information in their spec sheets! Usually, the first thing a program will do is convert the cHRM chromaticities into relative XYZ space.

What can I do with it?

If a PNG file has the gAMA and CHRM chunks, the source_RGB values can be converted to XYZ. This lets you:

* do accurate grayscale conversion (just use the Y component)

* convert to RGB for your own monitor (to see the original colors)

* print the image in Level 2 Postscript with better color fidelity than a simple RGB to CMYK conversion could provide

* calculate an optimal color palette

* pass the image data to a color management system

* etc. 
How do I convert from source_RGB to XYZ?

Make a few simplifying assumptions first, like the monitor really is jet black with no input and the guns don't interfere with one another. Then, given that you know the CIE XYZ values for each of red, green, and blue for a particular monitor, you put them into a matrix m:

$$
m=\begin{array}{lll}
X r & X g & X b \\
Y r & Y g & Y b \\
Z r & Z g & Z b
\end{array}
$$

Here we assume we are working with linear RGB floating point data in the range 0..1. If the gamma is not 1.0 , make it so on the floating point data. Then convert source_RGB to XYZ by matrix multiplication:

$$
\begin{aligned}
& \mathrm{X} \quad \mathrm{R} \\
& Y=m G \\
& \text { Z B }
\end{aligned}
$$

In other words, $\mathrm{X}=\mathrm{Xr} \mathrm{R}^{*}+\mathrm{Xg}{ }^{*} \mathrm{G}+\mathrm{Xb} \mathrm{B}^{\star}$, and similarly for $\mathrm{Y}$ and $\mathrm{Z}$. You can go the other way too:

$$
\begin{array}{ll}
R & X \\
G & =i m \\
B & Y \\
Z
\end{array}
$$

where im is the inverse of the matrix $\mathrm{m}$.

What is a gamut?

The gamut of a device is the subset of visible colors which that device can display. (It has nothing to do with gamma.) The gamut of an RGB device can be visualized as a polyhedron in XYZ space; the vertices correspond to the device's black, blue, red, green, magenta, cyan, yellow and white.

Different devices have different gamuts, in other words one device will be able to display certain colors (usually highly saturated ones) that another device cannot. The gamut of a particular RGB device can be determined from its R, G, and B chromaticities and white point (the same values given in the cHRM chunk). The gamut of a color printer is more complex and can only be determined by measurement. However, printer gamuts are typically smaller than monitor gamuts, meaning that there can be many colors in a displayable image that cannot physically be printed. 
Converting image data from one device to another generally results in gamut mismatches --- colors that cannot be represented exactly on the destination device. The process of making the colors fit, which can range from a simple clip to elaborate nonlinear scaling transformations, is termed gamut mapping. The aim is to produce a reasonable visual representation of the original image.

Further reading

References [COLOR-1] through [COLOR-5] provide more detail about color theory.

15. Appendix: Sample CRC Code

The following sample code represents a practical implementation of the CRC (Cyclic Redundancy Check) employed in PNG chunks. (See also ISO 3309 [ISO-3309] or ITU-T V.42 [ITU-V42] for a formal specification.)

The sample code is in the ANSI C programming language. Non C users may find it easier to read with these hints:

$\&$

Bitwise AND operator.

$\wedge$

Bitwise exclusive-OR operator. (Caution: elsewhere in this document, $\wedge$ represents exponentiation.)

$>>$

Bitwise right shift operator. When applied to an unsigned

quantity, as here, right shift inserts zeroes at the left.

!

Logical NOT operator.

$++$

"n++" increments the variable $n$.

OxNNN

Ox introduces a hexadecimal (base 16) constant. Suffix L indicates a long value (at least 32 bits).

/* Table of CRCs of all 8-bit messages. */ unsigned long crc_table[256];

/* Flag: has the table been computed? Initially false. */ int crc_table_computed $=0$;

Boutell, et. al.

Informational

[Page 94] 


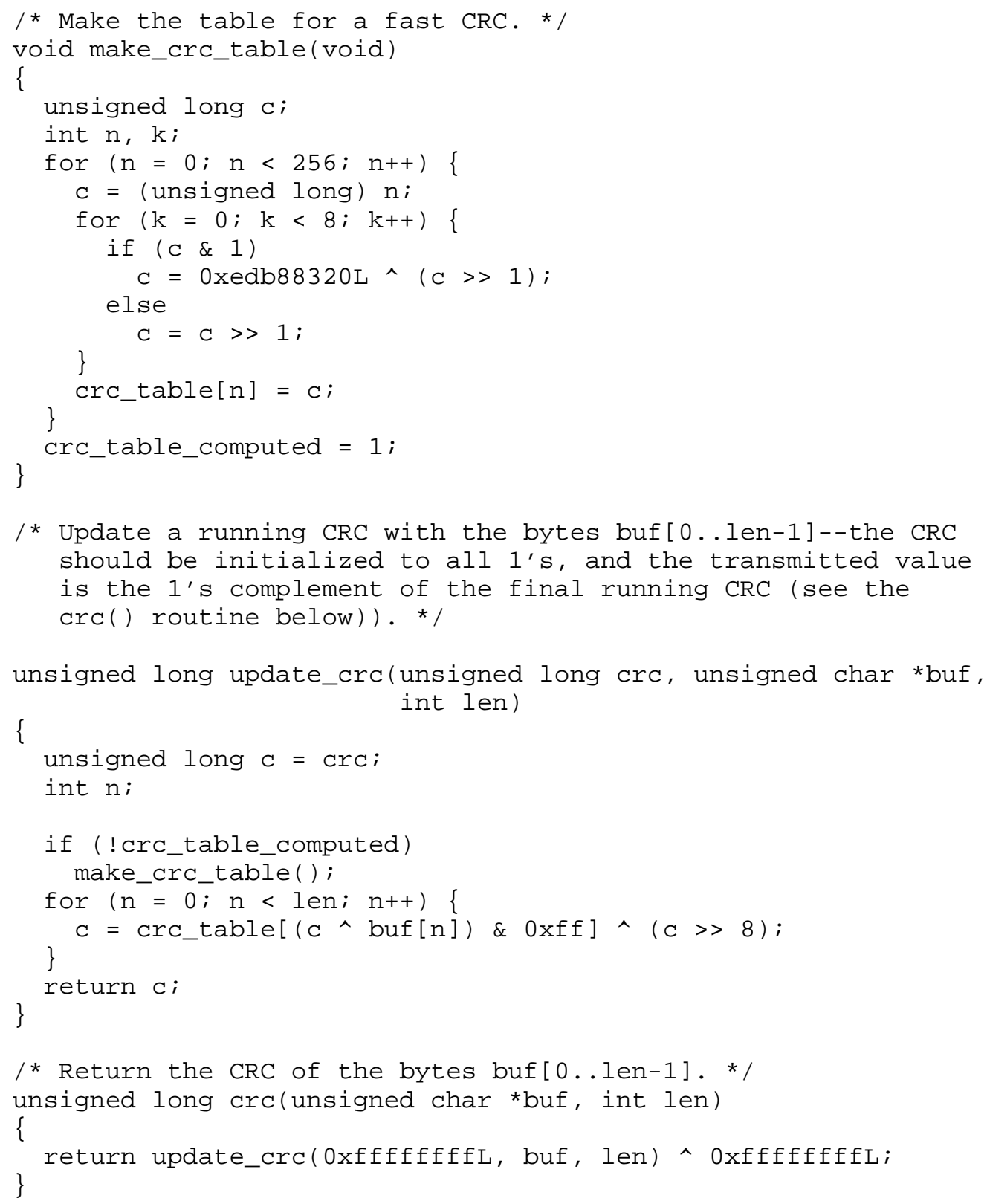


16. Appendix: Online Resources

(This appendix is not part of the formal PNG specification.)

This appendix gives the locations of some Internet resources for PNG software developers. By the nature of the Internet, the list is incomplete and subject to change.

Archive sites

The latest released versions of this document and related information can always be found at the PNG FTP archive site, ftp://ftp.uu.net/graphics/png/. The PNG specification is available in several formats, including HTML, plain text, and Postscript.

Reference implementation and test images

A reference implementation in portable $\mathrm{C}$ is available from the PNG FTP archive site, ftp://ftp.uu.net/graphics/png/src/. The reference implementation is freely usable in all applications, including commercial applications.

Test images are available from ftp://ftp.uu.net/graphics/png/images/.

Electronic mail

The maintainers of the PNG specification can be contacted by email at png-infoduunet.uu.net or at png-group@w3.org.

PNG home page

There is a World Wide Web home page for PNG at http://quest.jpl.nasa.gov/PNG/. This page is a central location for current information about PNG and PNG-related tools.

17. Appendix: Revision History

(This appendix is not part of the formal PNG specification.)

The PNG format has been frozen since the Ninth Draft of March 7, 1995, and all future changes are intended to be backwards compatible. The revisions since the Ninth Draft are simply clarifications, improvements in presentation, and additions of supporting material.

On 1 October 1996, the PNG specification was approved as a W3C (World Wide Web Consortium) Recommendation. 


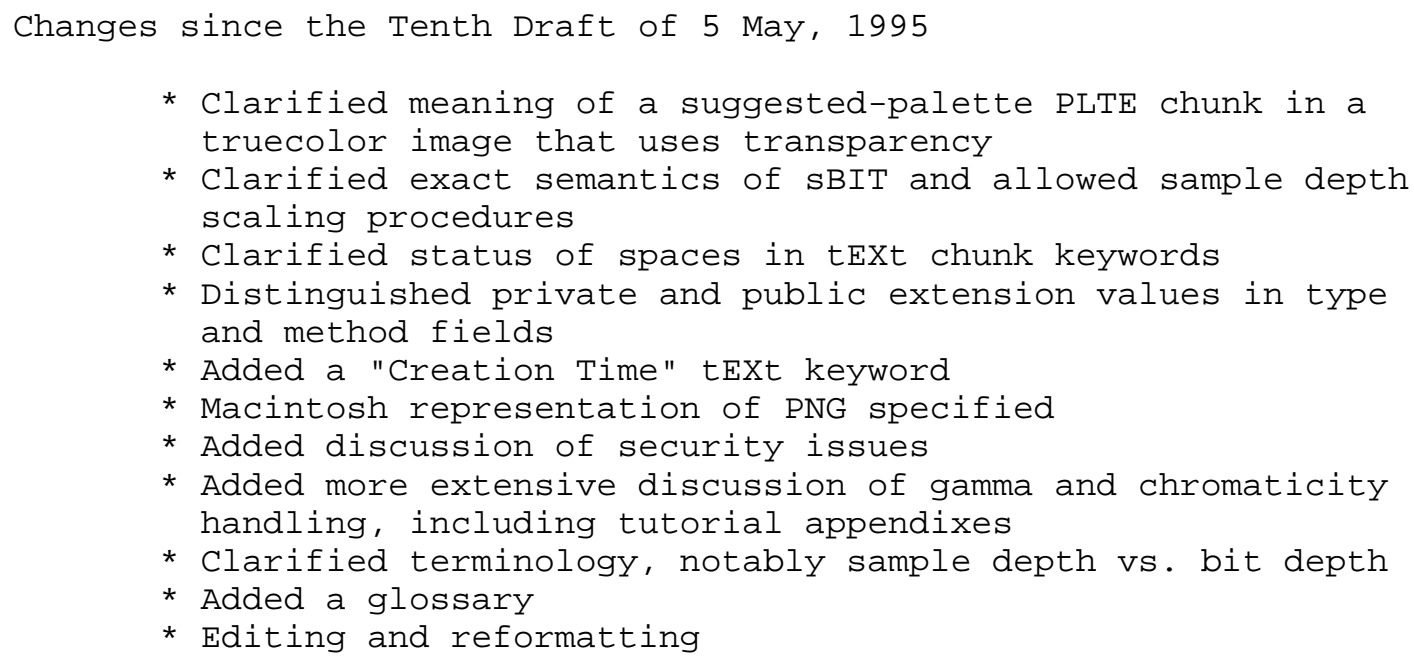

18. References

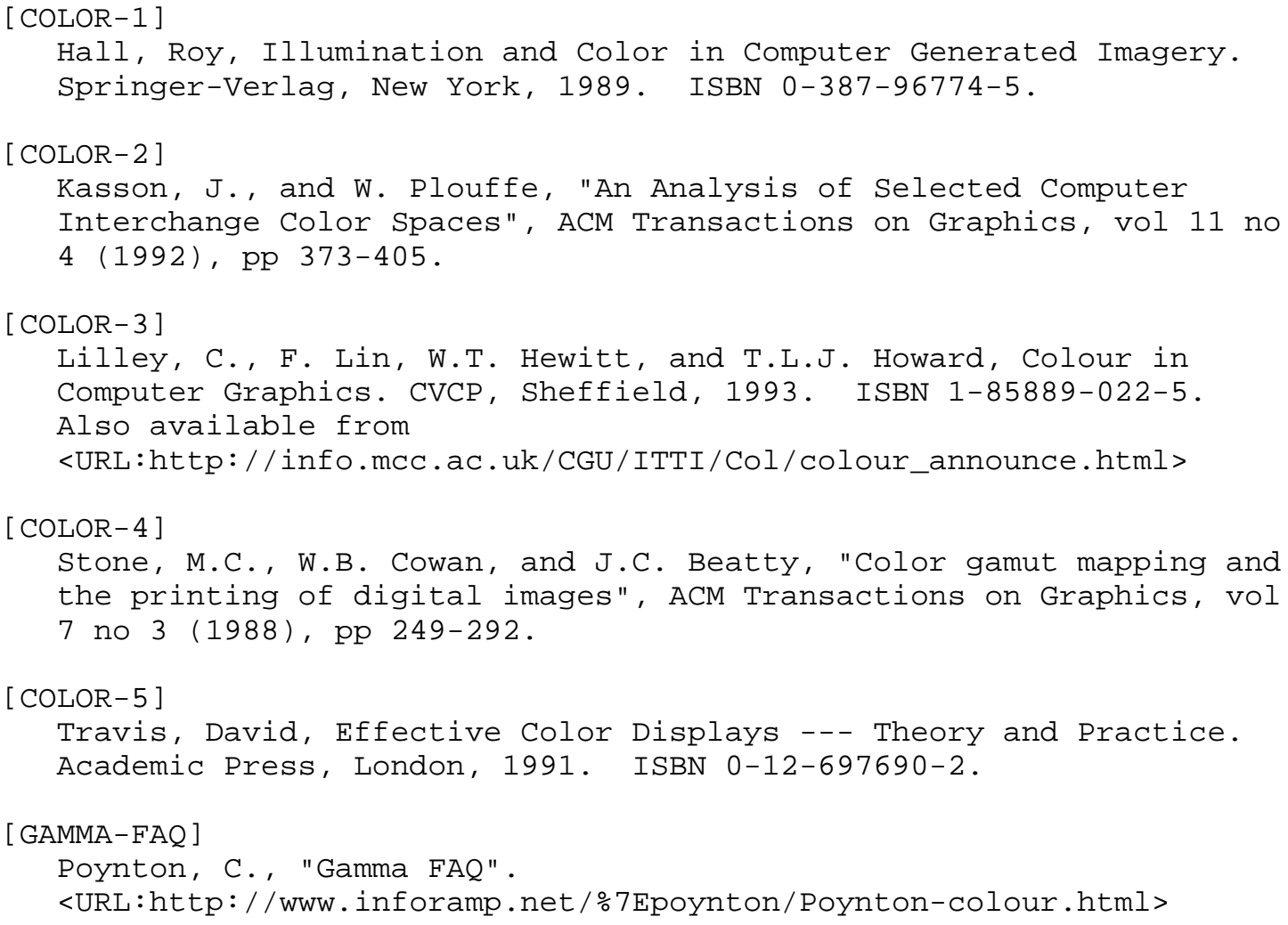




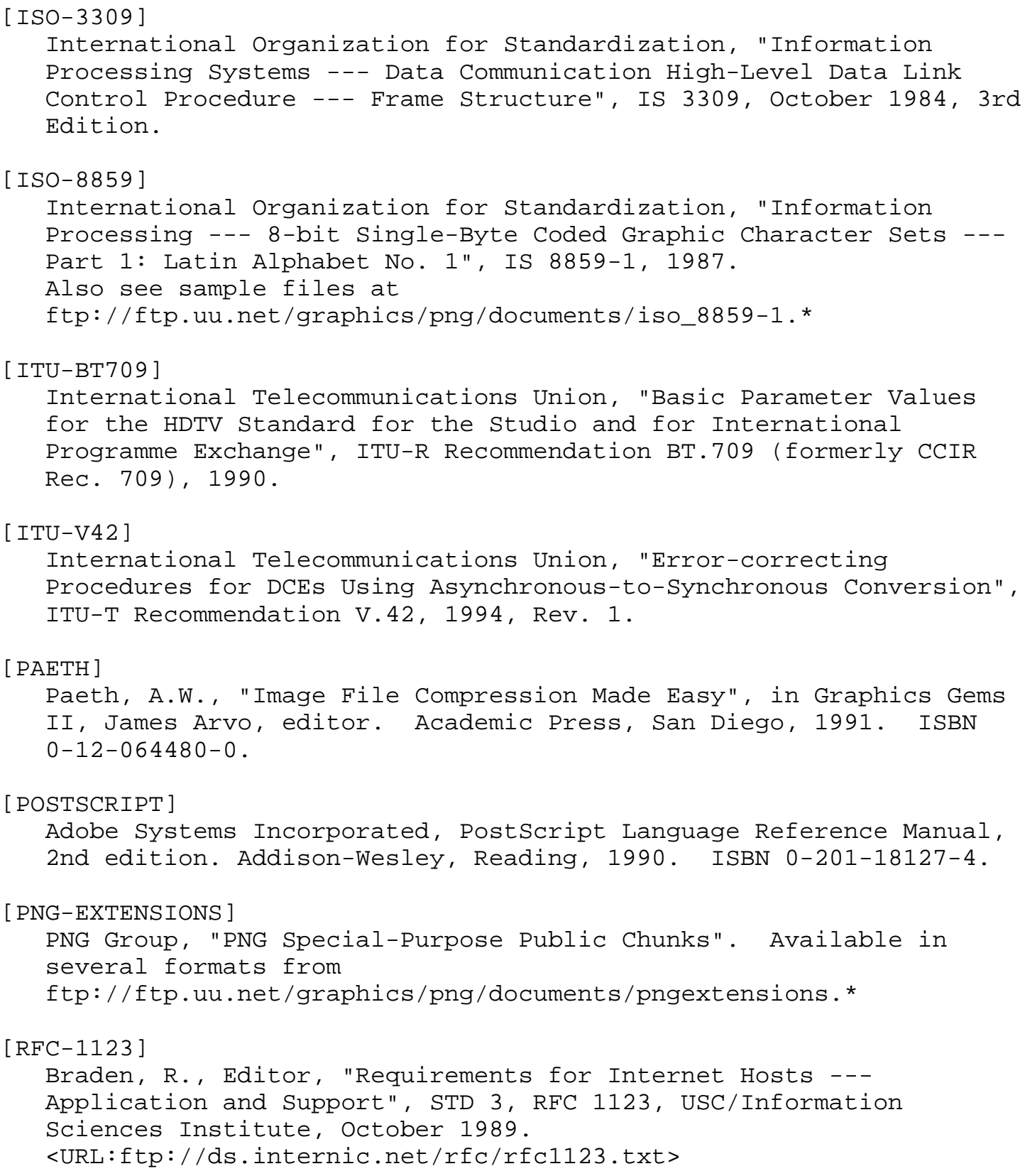




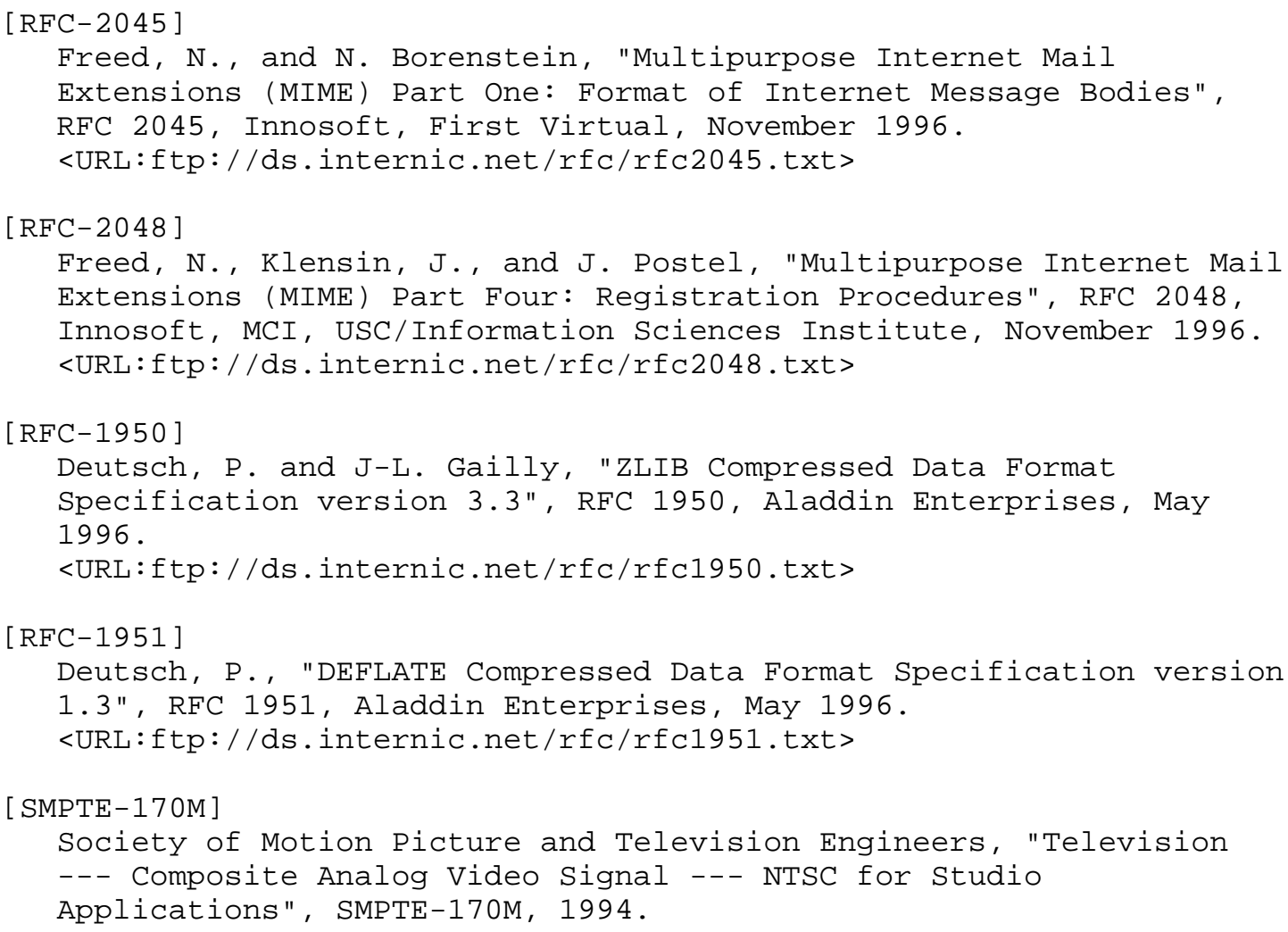


19. Credits

Editor

Thomas Boutell, boutell@boutell.com

Contributing Editor

Tom Lane, tglesss.pgh.pa.us

Authors

Authors' names are presented in alphabetical order.

* Mark Adler, madlerealumni.caltech.edu

* Thomas Boutell, boutelleboutell.com

* Christian Brunschen, cbedf.lth.se

* Adam M. Costello, amcles.berkeley.edu

* Lee Daniel Crocker, lee@piclab.com

* Andreas Dilger, adilger@enel.ucalgary.ca

* Oliver Fromme, frommedrz.tu-clausthal.de

* Jean-loup Gailly, gzipeprep.ai.mit.edu

* Chris Herborth, chrisheqnx.com

* Alex Jakulin, Aleks.Jakulin@snet.fri.uni-lj.si

* Neal Kettler, kettler@cs.colostate.edu

* Tom Lane, tgless.p.pgh.pa.us

* Alexander Lehmann, alexahal.rhein-main.de

* Chris Lilley, chris@w3.org

* Dave Martindale, davemecs.ubc.ca

* Owen Mortensen, 104707.650@compuserve.com

* Keith S. Pickens, kspeswri.edu

* Robert P. Poole, lionboyeprimenet.com

* Glenn Randers-Pehrson, glennrpearl.mil or randeg@alumni.rpi.edu

* Greg Roelofs, newt apobox.com

* Willem van Schaik, willemegintic.gov.sg

* Guy Schalnat

* Paul Schmidt, pschmidtephotodex.com

* Tim Wegner, 71320.675@compuserve.com

* Jeremy Wohl, jeremyw@anders.com

The authors wish to acknowledge the contributions of the Portable Network Graphics mailing list, the readers of comp.graphics, and the members of the World Wide Web Consortium (W3C). 
The Adam7 interlacing scheme is not patented and it is not the intention of the originator of that scheme to patent it. The scheme may be freely used by all PNG implementations. The name "Adam7" may be freely used to describe interlace method 1 of the PNG specification.

Trademarks

GIF is a service mark of Compuserve Incorporated. IBM PC is a trademark of International Business Machines Corporation. Macintosh is a trademark of Apple Computer, Inc. Microsoft and MS-DOS are trademarks of Microsoft Corporation. PhotoCD is a trademark of Eastman Kodak Company. PostScript and TIFF are trademarks of Adobe Systems Incorporated. SGI is a trademark of Silicon Graphics, Inc. X Window System is a trademark of the Massachusetts Institute of Technology.

COPYRIGHT NOTICE

Copyright (c) 1996 by: Massachusetts Institute of Technology (MIT)

This W3C specification is being provided by the copyright holders under the following license. By obtaining, using and/or copying this specification, you agree that you have read, understood, and will comply with the following terms and conditions:

Permission to use, copy, and distribute this specification for any purpose and without fee or royalty is hereby granted, provided that the full text of this NOTICE appears on ALL copies of the specification or portions thereof, including modifications, that you make.

THIS SPECIFICATION IS PROVIDED "AS IS," AND COPYRIGHT HOLDERS MAKE NO REPRESENTATIONS OR WARRANTIES, EXPRESS OR IMPLIED. BY WAY OF EXAMPLE, BUT NOT LIMITATION, COPYRIGHT HOLDERS MAKE NO REPRESENTATIONS OR WARRANTIES OF MERCHANTABILITY OR FITNESS FOR ANY PARTICULAR PURPOSE OR THAT THE USE OF THE SPECIFICATION WILL NOT INFRINGE ANY THIRD PARTY PATENTS, COPYRIGHTS, TRADEMARKS OR OTHER RIGHTS. COPYRIGHT HOLDERS WILL BEAR NO LIABILITY FOR ANY USE OF THIS SPECIFICATION. 
The name and trademarks of copyright holders may NOT be used in advertising or publicity pertaining to the specification without specific, written prior permission. Title to copyright in this specification and any associated documentation will at all times remain with copyright holders.

Security Considerations

Security issues are discussed in Security considerations (Section $8.5)$.

Author's Address

Thomas Boutell

PO Box 20837

Seattle, WA 98102

Phone: (206) 329-4969

EMail: boutellaboutell.com 